\title{
Aerodynamic and Aeroacoustic Analyses of Vertical-Axis Wind Turbines
}

by

Arvin Hashemi Mehr

\author{
A Thesis Submitted to the \\ Faculty of Graduate and Postdoctoral Affairs \\ in Partial Fulfillment of the Requirements for the Degree of \\ Master of Applied Science \\ in
}

Mechanical and Aerospace Engineering

Carleton University

Ottawa, Ontario

(C)Arvin Hashemi Mehr

August 2021 
The Undersigned Hereby Recommends to the Faculty of Graduate and Postdoctoral Affairs Acceptance of the Thesis

\section{Aerodynamic and Aeroacoustic Analyses of Vertical-Axis Wind Turbines}

\section{Submitted by Arvin Hashemi Mehr}

In Partial Fulfilment of the Requirement for the Degree of

\section{Master of Applied Science}

Professor Fred Nitzsche, Thesis supervisor

Professor Edgar Matida, Thesis supervisor

Ottawa-Carleton Institute for Mechanical and Aerospace

Engineering

Department of Mechanical and Aerospace Engineering

Carleton University

Ottawa, Ontario

(C)Arvin Hashemi Mehr

August 2021 


\section{Abstract}

In this thesis, aerodynamic and aeroacoustic analyses are performed for different configurations of Vertical Axis Wind Turbines (VAWTs). Performance of traditional Troposkien-Darrieus VAWTs and novel Shifted-Troposkien-Shape VAWTs (or STSVAWTs) with two configurations (50\% STS-VAWT and 100\% STS-VAWT) were simulated numerically. These novel VAWTs try to reduce the Blade-Wake Interactions (BWIs) between the turbine blades and the wake generated during revolutions, which are known to reduce performance of traditional Troposkien-Darrieus VAWTs. Separate experimental investigations (not included in this thesis) in the current laboratory, indicated that a $0.75-\mathrm{m} 50 \%$ STS-VAWT produced more power than a traditional $0.75-\mathrm{m}$ Troposkien-Darrieus VAWTs, while requiring less material to be manufactured. In the current investigation, simulations were performed using an in-house code (GENUVP, GENeral Unsteady Vortex Particle code), which was available as a part of a research collaboration with the University of Athens. Aerodynamic characteristics of VAWTs (mainly power generation as a function of wind speed at a fixed turbine rpm, or rotations per minute) can be obtained by computing the torque on the blades using unsteady panel methods in combination with particle-vortex methods. The numerical aerodynamic data become an input for the aeroacoustic part of the same in-house code. Aeroacoustic noise is predicted using the Formulation 1C of Ffowcs Williams - Hawkings equation. Numerical simulation results of power coefficient for 2-m and 17-m Troposkien-Darrieus VAWTs were initially validated against experimental data obtained from the literature. Additional simulations were also performed for 50\% STS-VAWT and 100\% STS-VAWT, confirming 
the attractive performance for the $50 \%$ STS-VAWT when power generation is concerned, although with an increase in acoustic noise downstream of the turbine. 


\section{Acknowledgements}

During my graduate studies, I was lucky to pursue my research under the supervision of Professors Fred Nitzsche and Edgar Matida. My Master's has been an incredible experience, and I owe a huge debt of gratitude to both of them, not just for their unwavering academic support, but also for providing me with so many fantastic opportunities.

Professors Vasilis Riziotis and John Prospathopoulos as well as other scholars at the University of Athens, Greece, have my undying appreciation for their support and verifying my results. Their assistance paved the path in my research.

No words can adequately convey my gratitude and appreciation to my family members. Their love has been a key source of spiritual support for me throughout my life.

Finally, I want to express my gratitude to everyone who has provided me with support and encouragement in order to help me reach my objective and successfully complete my graduate study. 


\section{Table of Contents}

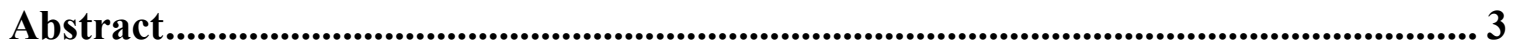

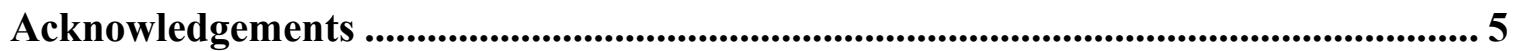

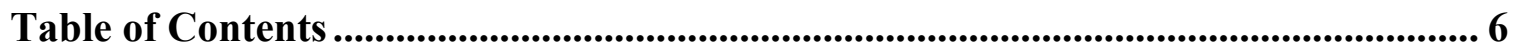

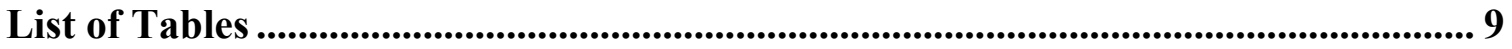

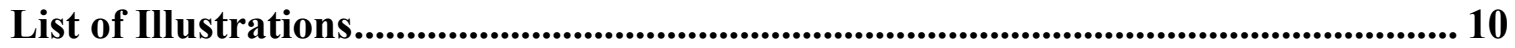

List of Appendices .................................................................................................................... 13

Chapter $1:$.............................................................................................................................. 20

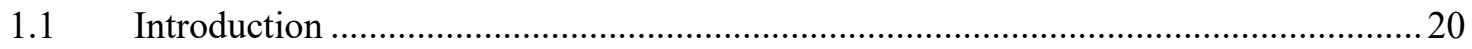

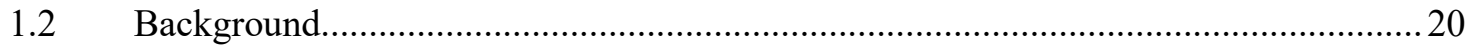

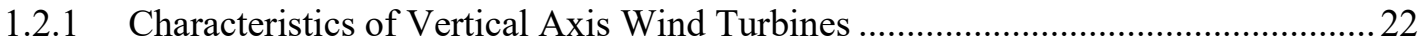

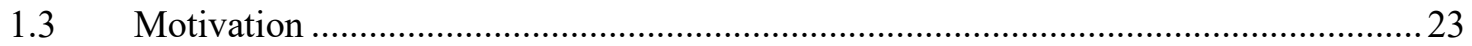

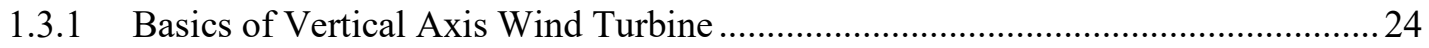

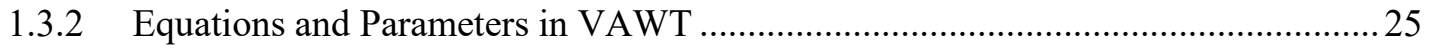

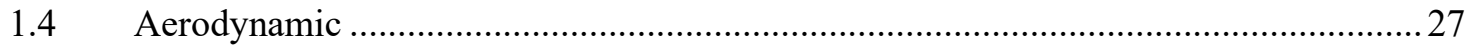

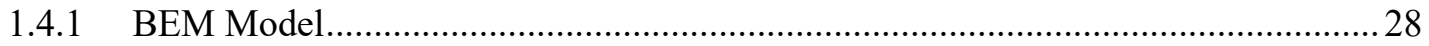

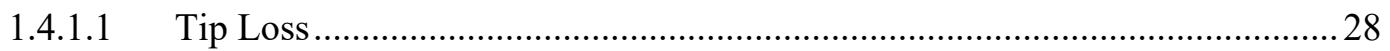

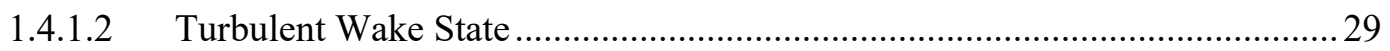

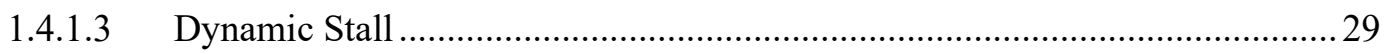

1.4.2 CFD Model (Computational Fluid Dynamics) Model ..........................................29

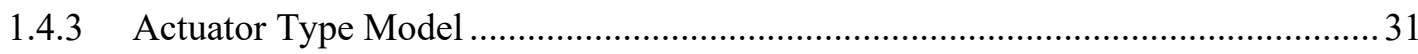

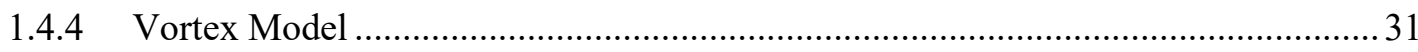

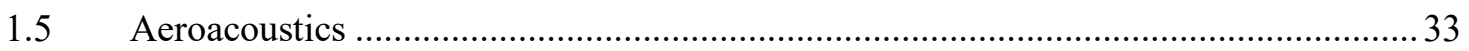

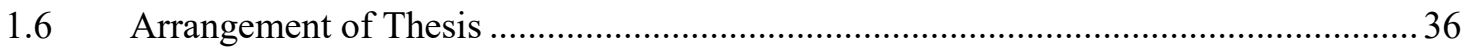

Chapter $2:$................................................................................................................................... 38 


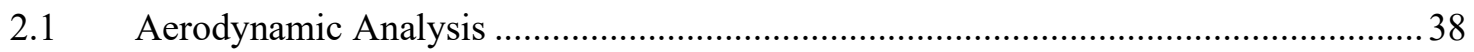

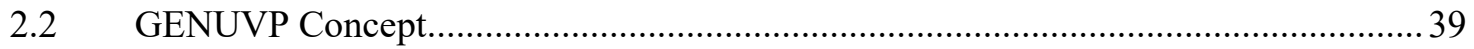

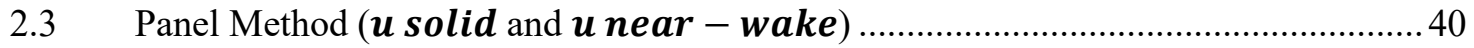

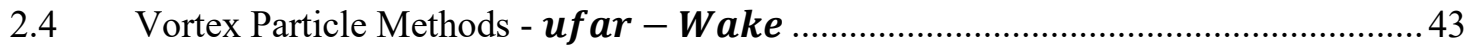

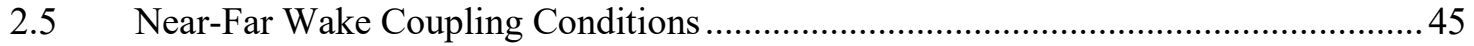

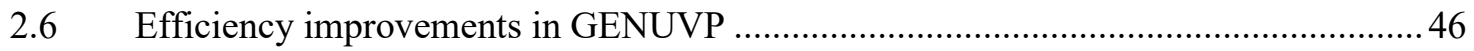

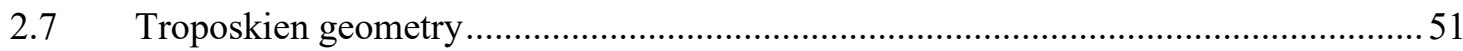

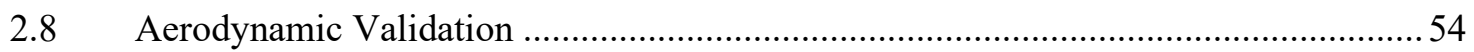

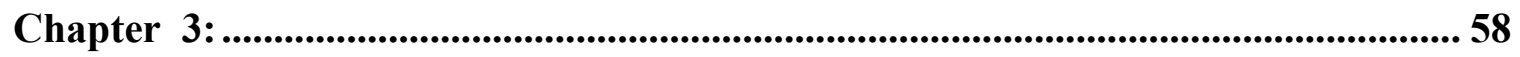

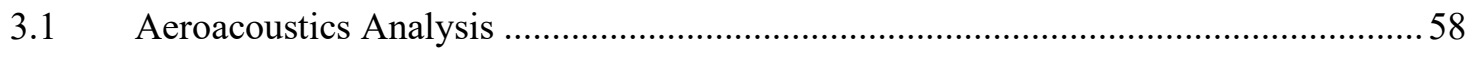

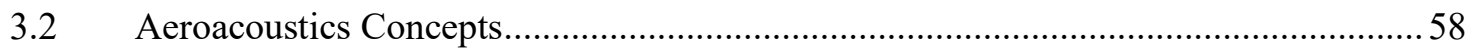

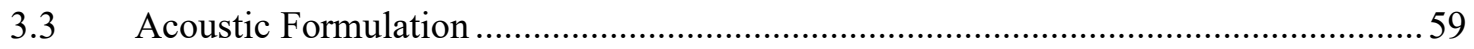

Chapter $4:$.................................................................................................................. 65

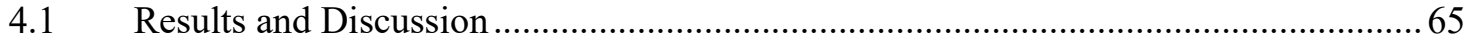

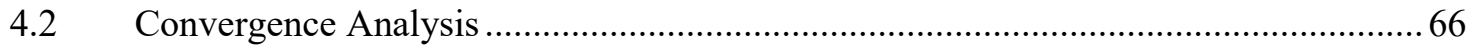

4.3 Aerodynamic Prediction of Different VAWT Models .................................................. 69

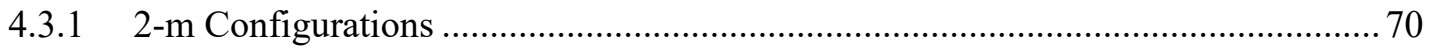

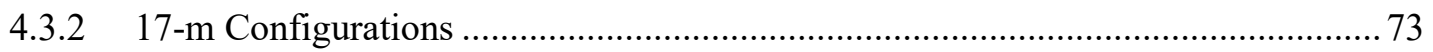

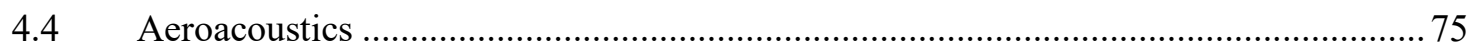

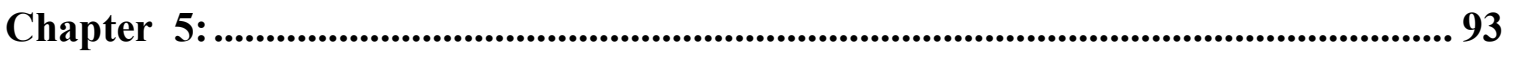

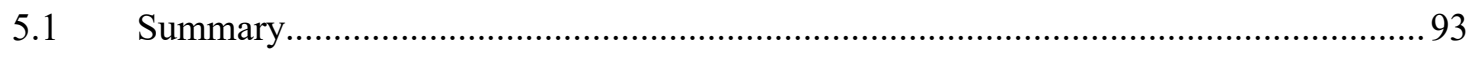

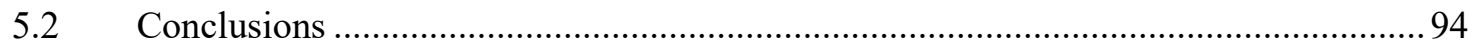

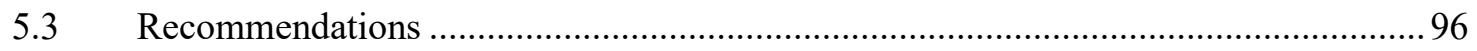

References ....................................................................................................................................... 97

Appendices.................................................................................................................................. 108 


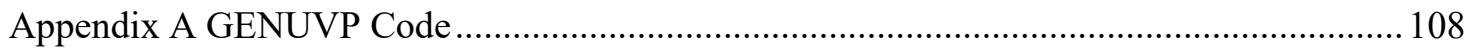

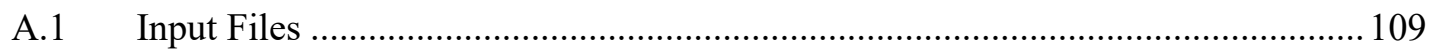

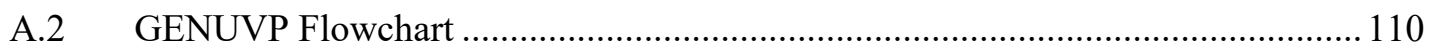

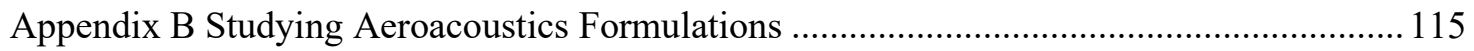

B.1 Origin of Formulation 1C for Non-Penetrable Surfaces .................................... 115 


\section{List of Tables}

Table 1 Geometric Characteristics of 2-m and 17-m Sandia VAWT .......................... 55

Table 2 Geometric Characteristics of 2-m Sandia STS-VAWT Configurations............ 71

Table 3 Geometric Characteristics of 17-m STS-VAWT.......................................... 73

Table 4 Geometric Characteristics for the 2-m VAWTs. ........................................... 76

Table 5 Geometric Characteristics for the 17-m VAWTs........................................ 80

Table 6 Geometric Characteristics for Different VAWTs Configurations.................... 84 


\section{List of Illustrations}

Figure 1 Availability of Wind in Different Parts of the World [2].............................. 20

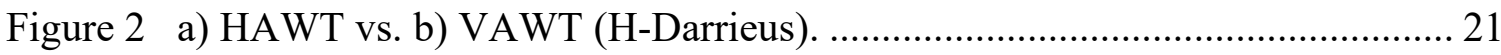

Figure 3 a) Savonius VAWT, and b) Troposkien-Darrieus VAWT............................... 22

Figure 4 Traditional Troposkien-Darrieus VAWTs and STS (Shifted Troposkien

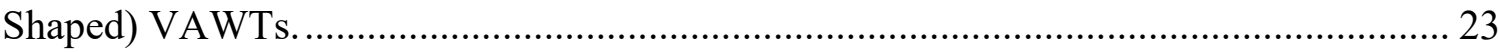

Figure 5 Blade Rotation (Not to Scale)............................................................................ 25

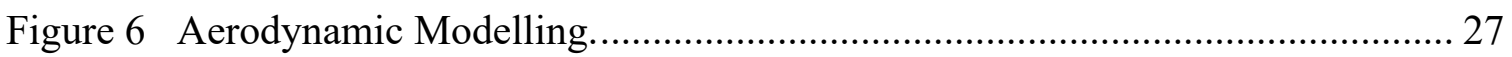

Figure 7 Vortex Particles Formation from a Trailing-Edge Wake Strip. ....................... 46

Figure 8 Grid Refinement Diagram for Different Levels Used for Subgrid

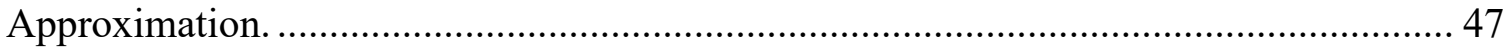

Figure 9 Top Level Block Diagram of GENUVP. ..................................................... 50

Figure 10 Conventional Troposkien Turbine with Some Geometric Parameters............ 52

Figure 11 Darrieus Turbine Performance Characteristics [5]..................................... 53

Figure 12 Performance Comparison of the SANDIA 2-m VAWT (27.9 rad/s)............ 55

Figure 13 Performance Comparison of the Sandia 17-m VAWT (5.5 rad/s)................ 56

Figure 14 Wake Generated by Rotation of a 2-m Sandia Conventional Design Turbine.57

Figure 15 Impact of the Azimuth Angle Increment on the Power Coefficient............... 66

Figure 16 Effect of the Turbine's Number of Revolutions on the Power Coefficient..... 67

Figure 17 Effect of the Number of Nodes in Each Blade, in Power Coefficient.............. 68

Figure 18 Effect of the Number of Panels on Each Blade Segment, in Power Coefficient. 
Figure 19 Geometry Schematics: a) Conventional Troposkien-Darrieus VAWT, b) 50\% STS-VAWT (Shifted-Troposkien-Shape VAWT), and c) 100\% STS-VAWT. 70

Figure 20 Turbine Performance for 2-m Conventional, 2-m 50\% STS-VAWT (Shifted Troposkien Shape - VAWT), and 2-m 100\% STS-VAWT at $27.9 \mathrm{rad} / \mathrm{s}$. 72

Figure 21 Turbine Performance for 2-m Conventional, 2-m 50\% STS-VAWT, and 2-m $100 \%$ STS-VAWT Configurations at $41.9 \mathrm{rad} / \mathrm{s}$. 72

Figure 22 Turbine Performance for 17-m Conventional, 17-m 50\% STS-VAWT (Shifted Troposkien Shape VAWT), and 17-m 100\% STS-VAWT at $4.4 \mathrm{rad} / \mathrm{s}$. 74

Figure 23 Turbine Performance for 17-m Conventional, 17-m 50\% STS-VAWT (Shifted Troposkien Shape VAWT), and 17-m 100\% STS-VAWT at $5.5 \mathrm{rad} / \mathrm{s}$. 74

Figure 24 Location of the Turbine and Plane where Acoustic Analysis is Performed.... 75 Figure 25 Acoustic Map at a Plane $2.5 \mathrm{~m}$ Downstream from the Axis of the Turbine: a) 2-m Conventional VAWT and b) 2-m 50\% STS-VAWT. 77 Figure 26 Exact Location of the Point that Acoustic Pressure for Different Frequencies is Calculated on, in the 2-m VAWT Configurations. 78

Figure 27 Acoustic Pressure vs. Frequency: a) 2-m Conventional VAWT and b) 2-m $50 \%$ STS-VAWT.

Figure 28 Acoustic Map at a Plane 2.5 Rt Downstream from the Axis of the Turbine: a) 17-m Conventional VAWT and b) 17-m 50\% STS-VAWT. 81

Figure 29 Exact Location of the Point that Acoustic Pressure for Different Frequencies is Calculated on, in the 17-m VAWT Configurations. 82 Figure 30 Acoustic Pressure vs. Frequency: a) 17m Conventional VAWT and b) 17m 50\% STS-VAWT. 83 
Figure 31 Schematic Showing Placement of Different Five Planes and the VAWT 84

Figure 32 Acoustic Map Analysis on Different Planes for the 2-m Sandia Conventional Design. a) Plane Located at the $5 \boldsymbol{R t}$ Upstream, b) Plane Located at the $2.5 \boldsymbol{R t}$ Upstream, c) Plane Located at the Turbine's Axis of Rotation, d) Plane Located at the 2.5

$\boldsymbol{R t}$ Downstream, e) Plane Located at the $5 \boldsymbol{R t}$ Downstream...................................... 86

Figure 33 Acoustic Map Analysis on Different Planes for the 2-m 50\% STS Design. a) Plane Located at the $5 \boldsymbol{R} \boldsymbol{t}$ Upstream, b) Plane Located at the $2.5 \boldsymbol{R} \boldsymbol{t}$ Upstream, c) Plane Located at the Turbine's Axis of Rotation, d) Plane Located at the $2.5 \boldsymbol{R t}$ Downstream,

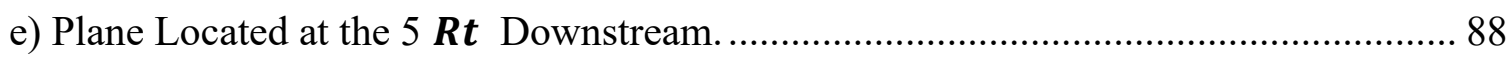

Figure 34 Acoustic Map Analysis on Different Planes for the 17-m Sandia Conventional Design. a) Plane Located at the $5 \boldsymbol{R t}$ Upstream, b) Plane Located at the $2.5 \boldsymbol{R t}$ Upstream, c) Plane Located at the Turbine's Axis of Rotation, d) Plane Located at the 2.5 $\boldsymbol{R t}$ Downstream, e) Plane Located at the $5 \boldsymbol{R t}$ Downstream. ...................................... 90 Figure 35 Acoustic Map Analysis on Different Planes for the 17-m 50\% STS Design. a) Plane Located at the $5 \boldsymbol{R t}$ Upstream, b) Plane Located at the $2.5 \boldsymbol{R} \boldsymbol{t}$ Upstream, c) Plane Located at the Turbine's Axis of Rotation, d) Plane Located at the $2.5 \boldsymbol{R t}$ Downstream,

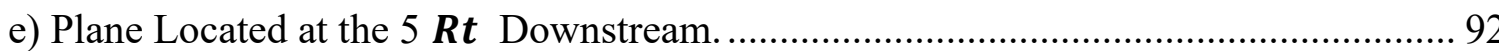




\section{List of Appendices}

Appendix A.......................................................... 106

A.1 Sub-Appendix..................................................... 109

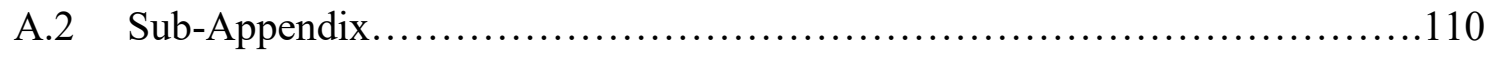

Appendix B. ............................................................. 115

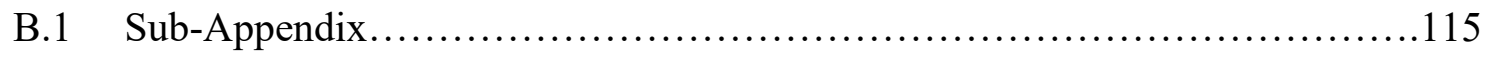




\section{List of Symbols}

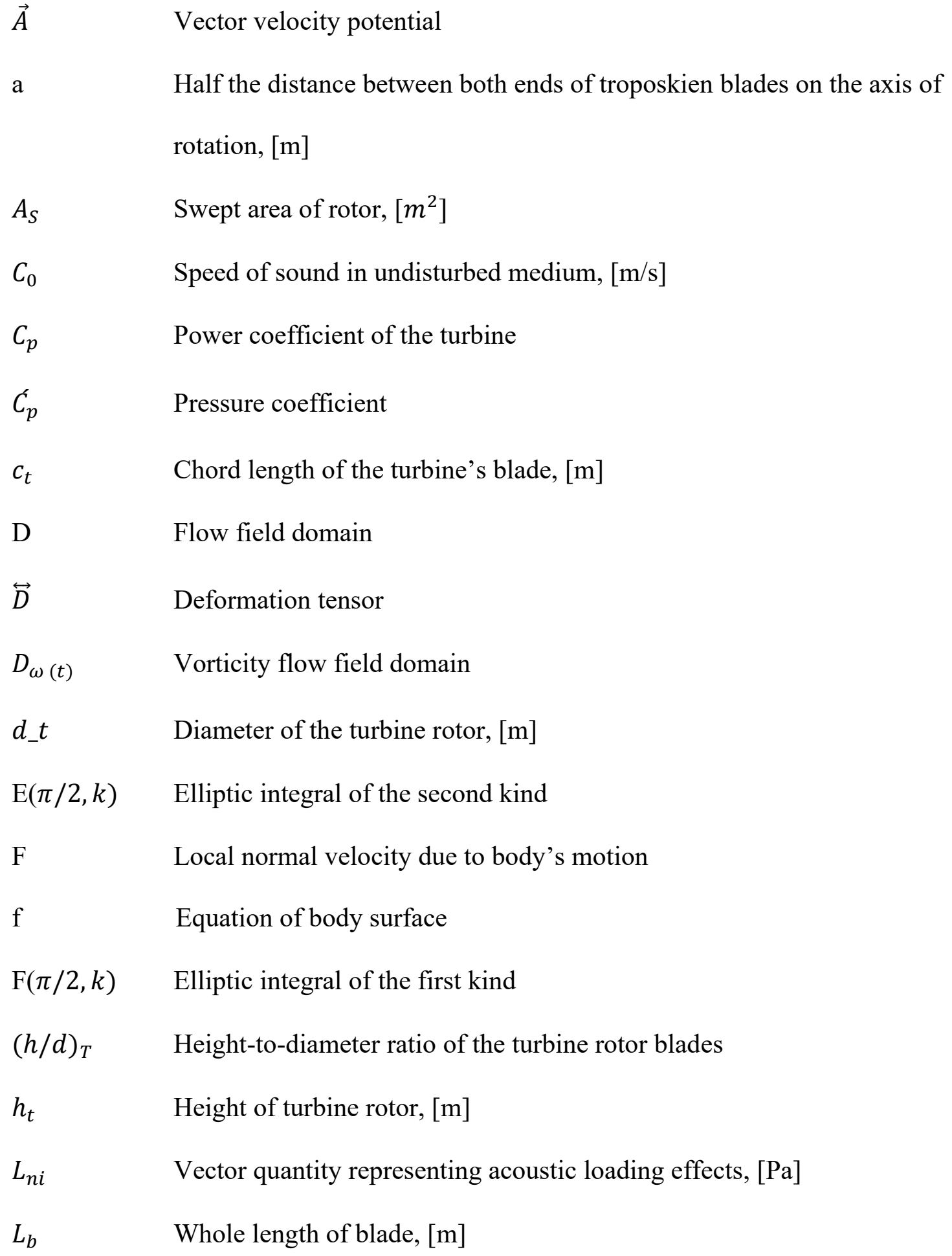




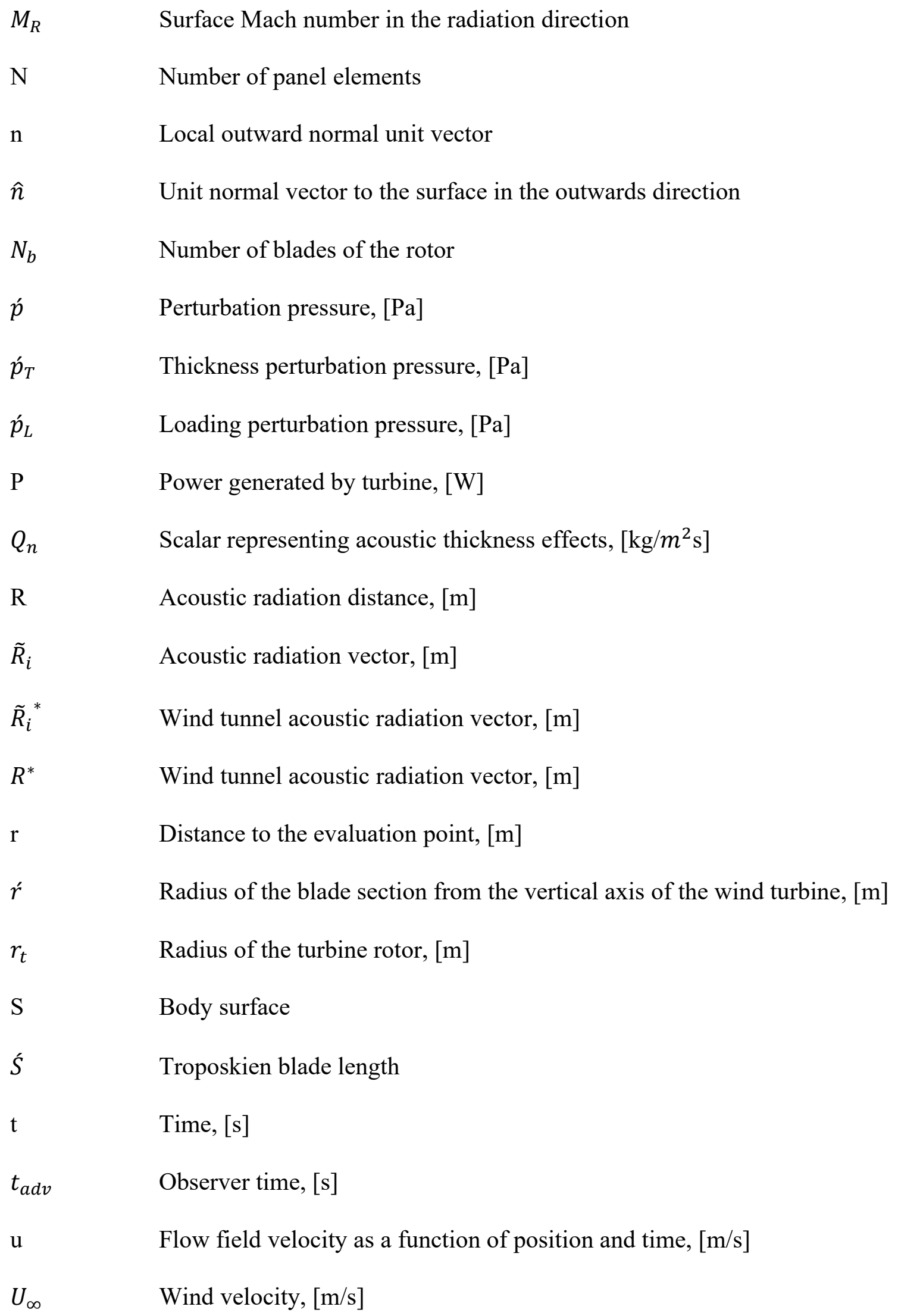


Position of the $\mathrm{j}$-th particle 


\section{Greek Symbols}

\begin{tabular}{|c|c|}
\hline$\beta$ & Ratio of the equator radius to half of the height of the wind turbine \\
\hline$\dot{\beta}$ & Prandtl-Glauert factor (Chapter 3) \\
\hline$\Omega$ & Vorticity, $\left[\mathrm{m}^{3} / \mathrm{s}\right]$ \\
\hline$\Omega_{t}$ & Turbine rotational velocity, $[\mathrm{rad} / \mathrm{s}]$ \\
\hline$\lambda_{t}$ & Turbine's rotor tip speed ratio \\
\hline$\tau_{e}$ & Emission source time, $[\mathrm{s}]$ \\
\hline$\omega$ & Vorticity, $\left[m^{3} / s\right]$ \\
\hline$\omega_{t}$ & Angular velocity of rotor, $[\mathrm{rad} / \mathrm{s}]$ \\
\hline$\sigma$ & Source intensity, $[\mathrm{m} / \mathrm{s}]$ \\
\hline$\sigma_{t}$ & Solidity ratio \\
\hline$\rho$ & Fluid density $\left[\mathrm{kg} / \mathrm{m}^{3}\right]$ \\
\hline$\phi$ & Scalar velocity potential, $[\mathrm{m} / \mathrm{s}]$ \\
\hline$\dot{\phi}$ & Local angle of troposkien geometry \\
\hline$\mu$ & Dipole intensity \\
\hline$\varepsilon$ & Blob cut off length, $[\mathrm{m}]$ \\
\hline$\theta$ & Azimuth angle, [rad] \\
\hline
\end{tabular}




\begin{tabular}{ll} 
Acronyms & \\
BEM & Blade Element Momentum \\
BET & Blade Element Theory \\
BL & Beddoes Leishman \\
BV & Boeing Vertol \\
BWI & Blade Wake Interaction \\
CFD & Computational Fluid Dynamics \\
DES & Detached Eddy Simulation \\
FW-H & Ffowcs Williams - Hawkings \\
GENUVP & GENeral Unsteady Vortex Particle \\
GW & Giga Watt \\
LES & Large Eddy Simulation \\
HAWT & Horizontal Axis Wind Turbines \\
PM & Particle Mesh \\
RANS & Reynolds Averaged Navier-Stokes \\
RMS & Root Mean Squared \\
SPL & Sound Pressure Level \\
TSR & Tip Speed Ratio \\
VAWT & Vertical Axis Wind Turbines \\
\hline
\end{tabular}




$\begin{array}{ll}(\vec{c}) & \text { Unit vector } \\ (\vec{c}) & \text { Vector } \\ (\sim) & \text { Dual matrix operator }\end{array}$




\section{Chapter 1:}

\subsection{Introduction}

Usage of renewable energy has increased significantly in the past decades due to higher energy demand, concerns regarding global warming and environmental pollution, as well as depletion of fossil fuel resources. Biomass, solar, hydroelectric, geothermal, and wind, among others, constitute different sources of renewable energy. Wind energy is relatively abundant in some parts of the world as shown in Figure 1, and its exploitation through power generation technology has been investigated extensively by several researchers [1].

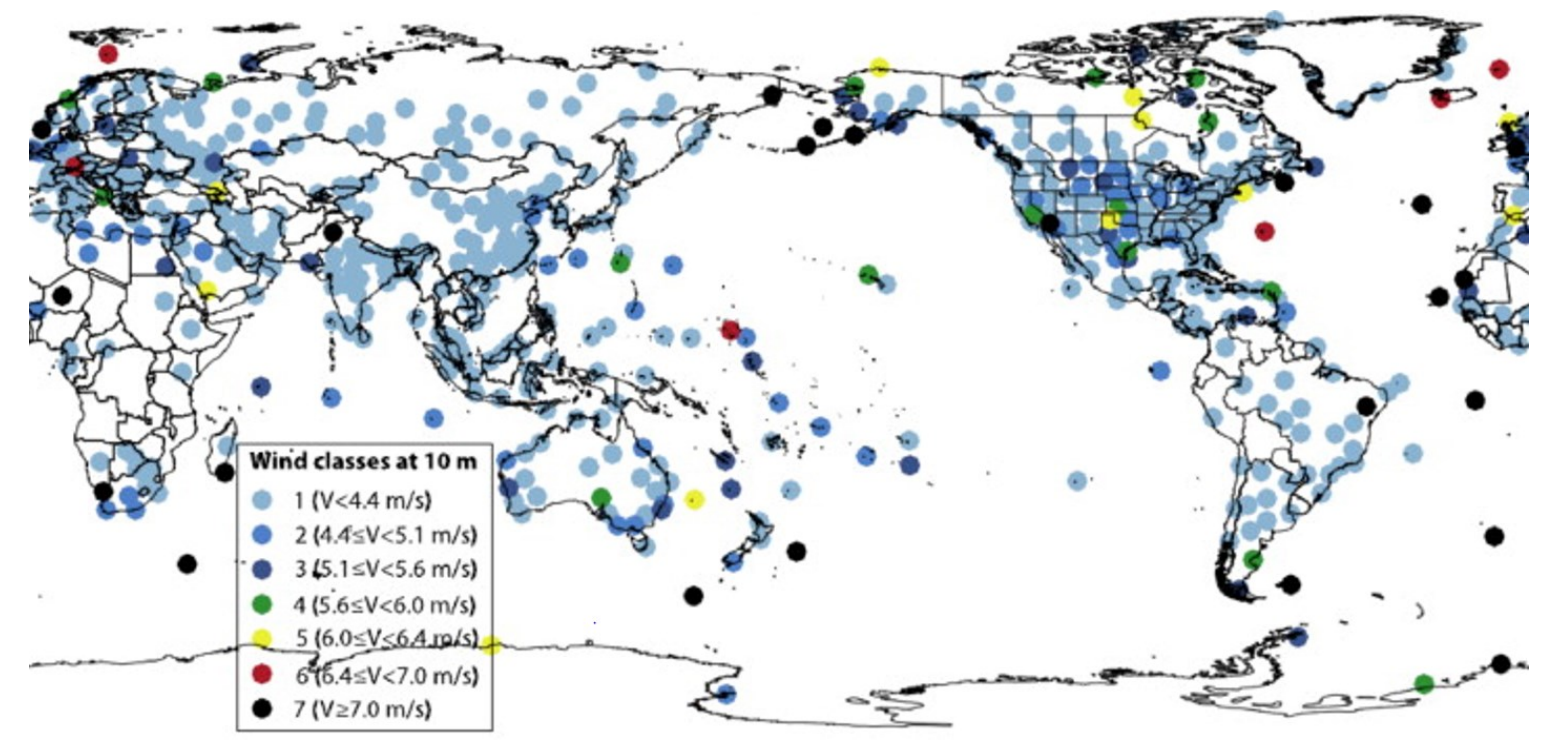

Figure 1 Availability of Wind in Different Parts of the World [2].

\subsection{Background}

Wind energy is intermittent, varying considerably throughout the year as well as during the day, with sporadic dangerous events (e.g., storms, tornados, and hurricanes). Wind has been explored since ancient times to propel sailing boats or ships, to grind grains and 
irrigate lands (windmills). Current technology allows wind to be converted to electric power through the usage of wind turbines [3].

Statistics from the World Wind Energy Association indicate 774 GW of installed wind power generation capacity in 2020 . Offshore installations represent approximately $5 \%$ of the total installations with projections to grow to $8.2 \%$ by the end of the decade [4].

Wind turbines can be categorized by either their axis of rotation (horizontal or vertical) or main driving force (drag or lift). In the traditional HAWTs (Horizontal Axis Wind Turbines), the rotational axis of the turbine is parallel to the direction of the wind, while for the Vertical Axis Wind Turbines (VAWTs), the rotational axis is perpendicular to the wind, as shown in Figure 2. Savonius VAWT (Figure 3.a) is an example of drag-type wind turbine. Due to limitations regarding maximum power coefficients and the relatively large quantity of material required for the rotors, Savonius drag-driven VAWTs are normally used for small-sized applications [5]. Troposkien-Darrieus VAWTs, as shown in Figure 3.b, are examples of turbines mostly driven by lift force, which are suited for large scale applications.

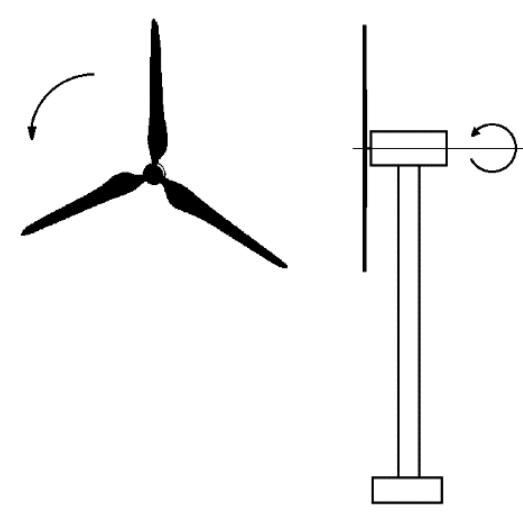

(a)

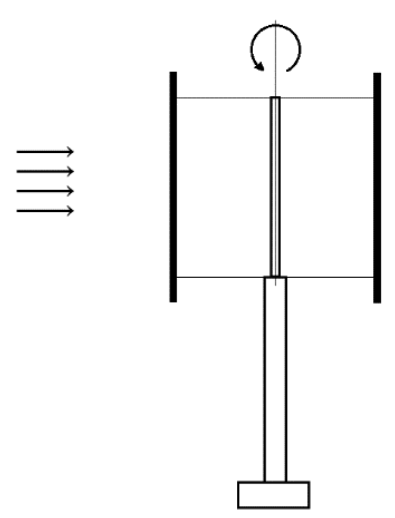

(b)

\section{Figure 2 a) HAWT vs. b) VAWT (H-Darrieus).}




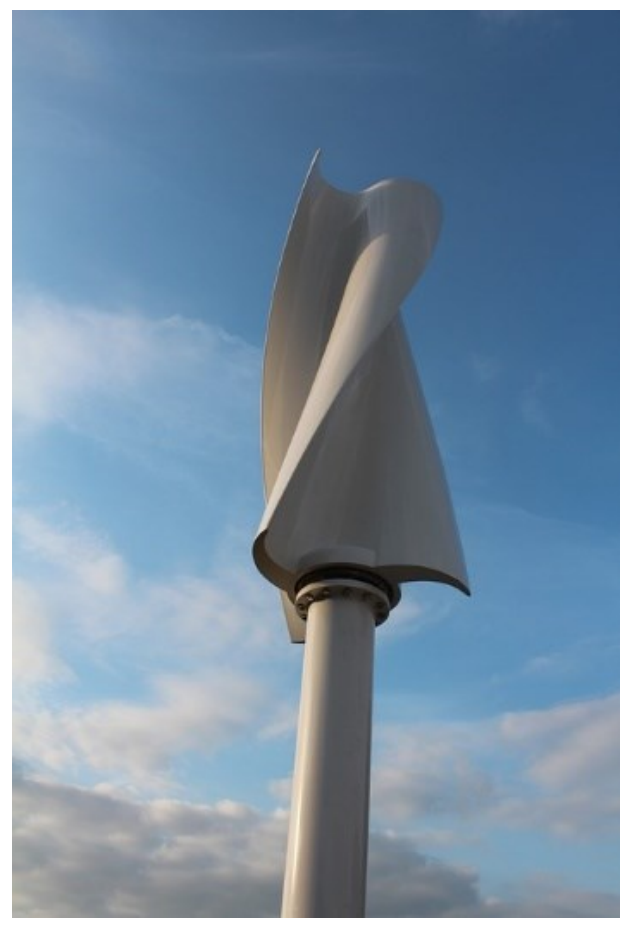

(a)

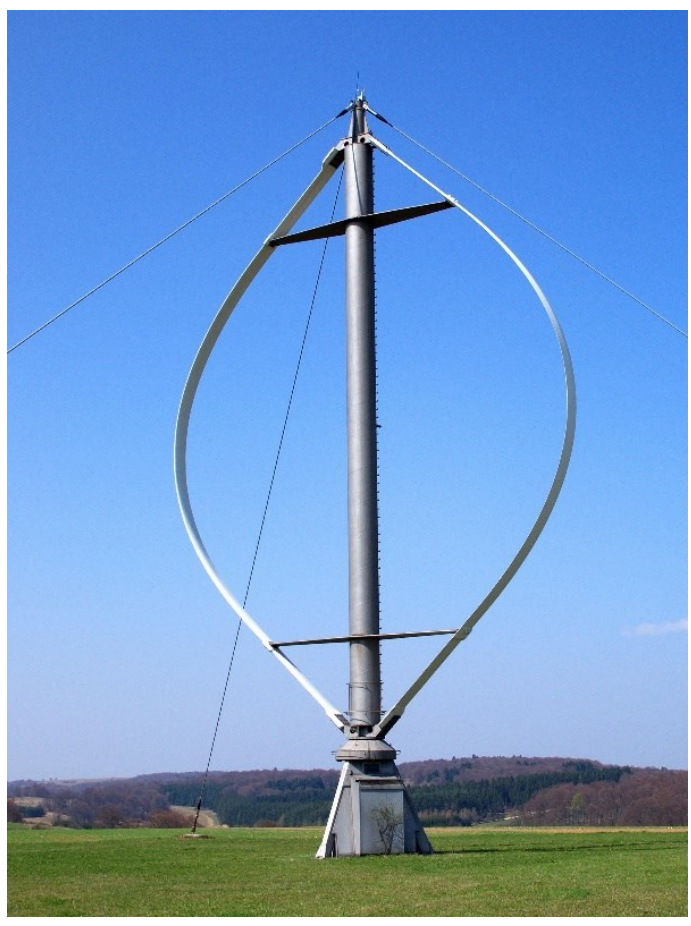

(b)

Figure 3 a) Savonius VAWT, and b) Troposkien-Darrieus VAWT.

\subsubsection{Characteristics of Vertical Axis Wind Turbines}

H-Darrieus, Savonius, and Troposkien-Darrieus have their own advantages and disadvantages. For example, Troposkien-Darrieus VAWTs have better performance in comparison to other VAWTs, but they are not self-starting turbines. In contrast, H-Darrieus VAWTs have self-starting ability. The Savonius turbines, which was invented in 1922 by the Finnish engineer Sigurd Johannes Savonius, have two or more curved-shape structures that experience less drag when moving against the wind in comparison to moving in direction of the wind [6]. Savonius turbines also have self-starting ability.

VAWTs were introduced commercially during the 1980s in California, where more than 500 turbines were installed by FloWind Corporation. Due to the unsteady-load nature 
of the system, blades made from extruded aluminum suffered a high failure rate due to material fatigue, thus VAWTs slowly disappeared from the wind power market [7], while HAWTs became the dominant mainstream configuration. It is worth mentioning that VAWT blades normally cost more than HAWT blades with an equivalent rotor swept-area [5], in addition to requiring a starting-system [7] [8]. Despite the disadvantages, there has been growing interest regarding VAWTs recently. For offshore applications with floating platforms, VAWTs are more stable than conventional HAWTs, becoming once again a viable alternative.

\subsection{Motivation}

To improve the performance of the Darrieus-Troposkien VAWT, a new concept was introduced in the current laboratory: $50 \%$ and 100\% Shifted-Troposkien-Shape VAWT [9], as shown in Figure 4.

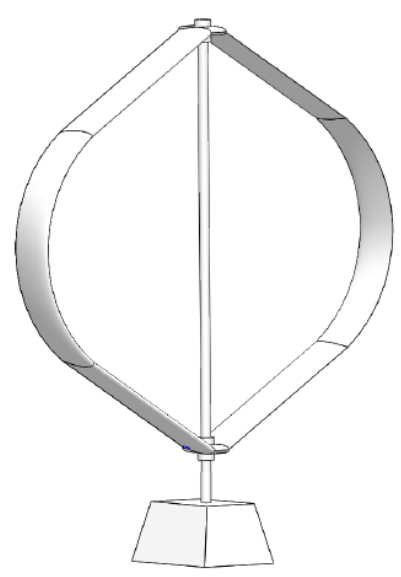

(a) Troposkien-Darrieus VAWT

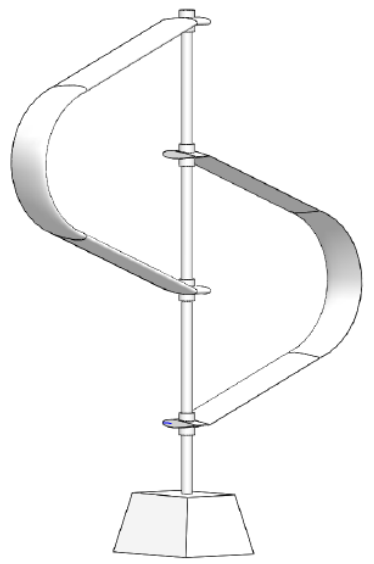

(b) $50 \%$ STS-VAWT

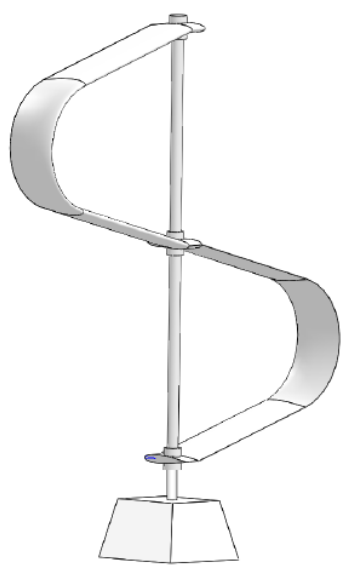

(c) $100 \%$ STS-VAWT

Figure 4 Traditional Troposkien-Darrieus VAWTs and STS (Shifted Troposkien Shaped) VAWTs. 
In the current investigation, an available in-house code will be used to simulate the Troposkien-Darrieus acoustic noise [10] and power generation efficiency (power coefficient as function of Tip Speed Ratio (TSR), parameters which will be defined later), with validations against experimental data available in the literature (2-m and 17-m VAWT, Sandia National Laboratories). New additional simulations will be performed for the $50 \%$ STS-VAWT, while keeping the same swept area and solidity as the $17-\mathrm{m}$ Troposkien-Darrieus Sandia VAWT. Aerodynamic performance of the STS model has been studied before in the current laboratory [9], but solidity ratio considerations were not taken into account when the performance of the turbines was studied. An important motivation of the current study include maintaining the solidity constant in both aerodynamic and aeroacoustic study of both conventional and STS models. Although not audible, wind turbines create infrasonic acoustic noise, that can cause not well-understood health effects in humans and impact wildlife [11] [12].

\subsubsection{Basics of Vertical Axis Wind Turbine}

In order to have a better understanding of a VAWT operation, consider a symmetric airfoil with a chord length of $c_{T}$ and a constant angular velocity of $\Omega_{T}$, rotating around a point with a circular distance of $r_{T}$, where $r_{T}$ is greater than $c_{T}$, as shown in Figure 5.

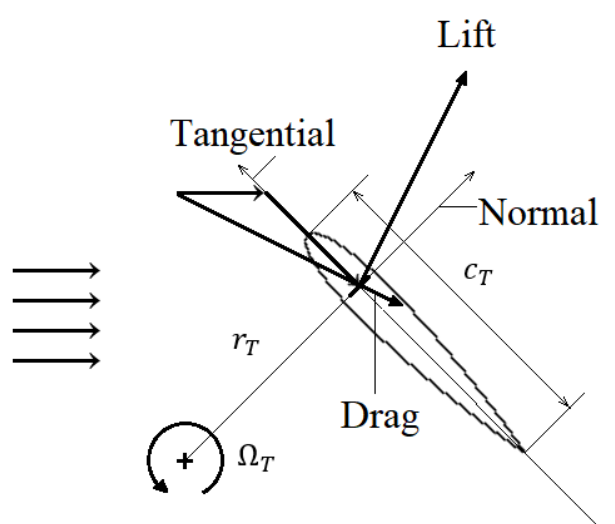




\section{Figure 5 Blade Rotation (Not to Scale).}

In a symmetric airfoil, we can assume that the angle of attack is tangent to its path of motion when rotating in a still medium; in this case, lift force would not be generated. On the other hand, if the same airfoil rotates in a uniform wind flow, the resultant velocity vector (from rotation and the uniform flow) will create an angle of attack, thus generating lift and drag forces, which create a torque about the axis of rotation.

There is an undistributed curvilinear flow around the rotor blades which is mainly because of the blade's curved path of motion. This curvilinear flow generates a virtual incidence angle which can add a negative offset to the geometric angle of attack that makes the effective angle of attack more negative at upwind azimuth angles and less positive at downwind azimuth angles.

There is more unsteadiness which a VAWT can experience, for example, blades can go through the wake generated by other blades, tower and even its own wake, which can have a direct impact on the relative flow around the blades and the angle of attack. In situations where the flow around the blades is not steady, the angle of attack alone is not a dependable criterion for flow prediction around the blades.

\subsubsection{Equations and Parameters in VAWT}

Some parameters and equations used for defining the characteristics of wind turbines, both VAWTs and HAWTs, will be defined here. TSR is the ratio of the velocity at the point with the highest rotational velocity (at the rotor's tip for HAWTs or at the equator plane for VAWTs) to the wind velocity (freestream velocity) and can be defined as follows: 


$$
\lambda_{T}=\frac{r_{T} \omega_{T}}{U_{\infty}},
$$

where $r_{T}$ is the rotor radius between the axis of rotation and the outermost point of the rotor blade, $\omega_{T}$ is the rotor angular velocity, and $U_{\infty}$ is the wind velocity. Solidity is one of the most important parameters in VAWTs. Solidity describes a relationship between the blade area and turbine swept area and can be shown as follows:

$$
\sigma_{T}=\frac{N_{b} c_{T} L_{b}}{A_{S}},
$$

where $N_{b}$ is the number of rotor blades, $c_{T}$ is the chord length, which is constant along the span for each blade (for VAWTs), $L_{b}$ is the whole length of the blade, and $A_{S}$ is the swept area. The swept area can be defined as the projection of the revolving turbine on a wind cross-section (or total frontal area of the revolving turbine). The height-to-diameter ratio can also be calculated as:

$$
\left(\frac{h}{d}\right)_{T}=\frac{h_{T}}{2 r_{T}} .
$$

Finally, power coefficient can be calculated using the following equation:

$$
C_{p}=\frac{P}{\frac{1}{2} A_{s} \rho U_{\infty}^{3}},
$$

where $P$ is the average power generated by the turbine in time, $\rho$ is the fluid density, $A_{s}$ is the swept area, and $U_{\infty}$ is the wind velocity.

In this thesis, the aerodynamic and aeroacoustic aspects of VAWTs are studied. Aeroacoustic noise can be defined as a sound that is created aerodynamically because of turbulent flow and its interaction with the object immersed in the flow [13] [14]. Accurate aeroacoustic results cannot be obtained without detailed information about the 
aerodynamics of the flow. The aerodynamics and aeroacoustics in VAWTs will be described in the following sub-sections.

\subsection{Aerodynamic}

To have precise aeroacoustic prediction of VAWTs, detailed aerodynamic information is required. The immersed object "thickness source" in aeroacoustics can be calculated only if the velocity distribution around the surface of the body is known. Pressure distribution over the body surface is also required in the calculation of the "loading source" in aeroacoustics. Hence, both detailed velocity and pressure distribution values are required for aeroacoustic prediction.

There are generally four types of aerodynamic models that can be used in wind turbine analysis, namely: 1) BEM model, 2) CFD (Computational Fluid Dynamics), 3) Actuator type model, and 4) Vortex model.

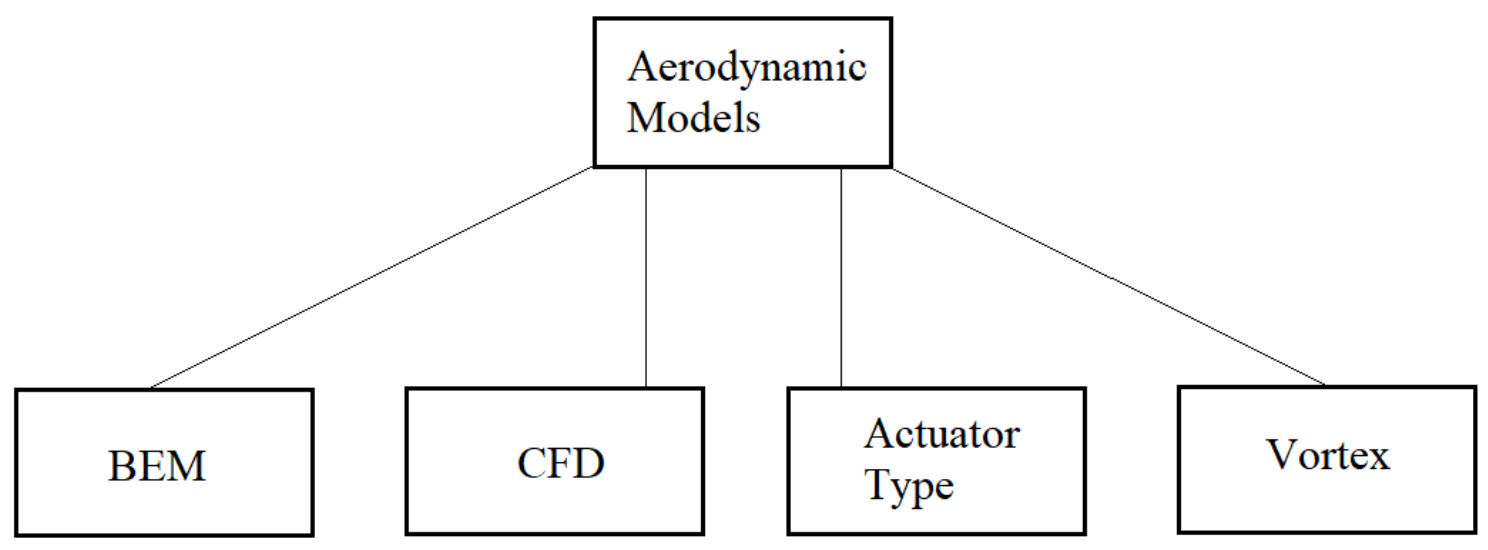

Figure 6 Aerodynamic Modelling. 


\subsubsection{BEM Model}

BEM (Blade Element Momentum) model is a combination of BET (Blade Element Theory) and blade momentum theory proposed by Glauert [15]. BET divides the blade into several elements and does not take into the account the mutual interactions between two adjacent components. Blade momentum theory introduces the axial and angular induction factors to calculate the generated axial and tangential velocities; hence combining these two models can better predict the performance parameters in each blade through an iterative process [16] [17]. The original BEM model suffers from some limitations regarding wind turbine applications, but most of them can be addressed using semi-empirical corrections. In the following sections, some of the limitations of the original BEM model are described.

\subsubsection{Tip Loss}

The first limitation of the original BEM model is the lack of tip loss modelling. BEM ignores the effects of vortices coming off the blade tip for HAWTs or H-Darrieus VAWTs. These vortices have a significant role in the induced velocity distribution along the blade, mainly near the blade tip area. Several tip loss factors are suggested to rectify this weakness. Prandtl proposed a tip loss correction factor by modeling the wind turbine's wake as vortex sheets [18]. This correction factor enhances the accuracy in the prediction of the induced velocity behavior. Another correction factor suggested by Xu and Sankar is based on Navier-Stokes solutions. This correction factor adjusted the Prandtl model, thus giving good results in a comparison against experimental data [19] [20]. 


\subsubsection{Turbulent Wake State}

Additional shortcoming of the original BEM model was its inability to perform well, when axial induction factors exceeded 0.4, normally occurring at high TSRs (Tip Speed Ratios) when blades interact with turbulent wakes. In such situations, original BEM models could not generate precise thrust coefficients. Several empirical models were suggested to solve this problem (for example, AeroDyn model [21], GH-Bladed model [22], Buhl model [23], Glauert model [24], and Burton model [25]).

\subsubsection{Dynamic Stall}

Dynamic stall is an aerodynamic effect that is related to the separation of the boundary layer. Dynamic stall happens when the angle of attack changes quickly over the airfoil. Dynamic stall initiates when the boundary layer starts to separate at the trailing edge and slowly moves to the leading edge, while the angle of attack is increased. Although the presence of the dynamic stall can be beneficial for the power generation of wind turbines [26], this phenomenon can cause some issues as well. Blade aeroelastic vibrations can be generated and increase the blade fatigue due to unsteady forces [27]. Some dynamic stall models are available in the literature, such as the ONERA model [28], BV (Boeing-Vertol) model [29], Snel model [30], BL (Beddoes- Leishman) model [31], and Risø [32].

\subsubsection{CFD Model (Computational Fluid Dynamics) Model}

CFD modelling is another approach that can be used in the aerodynamic analysis of wind turbines. This model normally uses the Unsteady Reynolds Averaged Navier-Stokes (URANS) equations to solve the flow around the blade. These equations are used in a 
discretized numerical domain and solved iteratively. Although this model does not need the blade's predetermined aerodynamic information, three-dimensional geometries of the blades need to be clearly described. Also, due to the complexity of the blade geometry, the creation of an appropriate mesh is generally difficult. Three main mesh techniques can be used in the CFD model. These models are structured, unstructured, and hybrid mesh techniques. Structured mesh has some advantages over the other models; for example, it is known as a high-resolution mesh, easily converged, while requiring relatively less memory usage, but it is challenging to apply the structured mesh on a complex geometry like the turbine blade. Unstructured mesh on the other hand, can be used easily for complex geometries, but it needs a higher computational time. Hybrid mesh is a mixture of structured and unstructured mesh techniques. In this approach, structured mesh is applied in the important parts of geometry like the boundary layers, and unstructured mesh is used in other parts, providing flexibility [33] [34] [35].

For wind turbine applications, relative wind speed around the wind turbine blades is normally much lower than the speed of sound, therefore, flow can be considered incompressible [36]. Modern HAWTs can have diameters in excess of $100 \mathrm{~m}$ in which compressibility effects may become important close to the tip of the rotor blades. Different RANS-based turbulence models are utilized in the wind turbine field, for example, SST (Shear Stress Transport) model [37], k- $\varepsilon$ model [38], and k- $\omega$ model. It has been reported that $\mathrm{k}-\omega$ based SST model is one of the best models that can be used in both $2 \mathrm{D}$ and $3 \mathrm{D}$ blade CFD modelling [39] [40] [41]. 


\subsubsection{Actuator Type Model}

This model represents wind turbine blade as a surface, disk or line with the corresponding load distribution on the surface, disk or line. There are different types of actuator disk models which are categorized by representation of the blade, i.e., actuator disk model, actuator surface model, and actuator line model. Actuator disk model is recognized as the first model which was used to study rotor's performance. This model is obtained from onedimension theory which was suggested by Rankine [42] and Froude [43] that culminated into the Blade Element Theory model [15]. This model can also be linked to the NavierStokes or Euler numerical solutions. Later on, the non-uniformly loaded actuator disc method was expanded by Sorensen and Shen to form the actuator line approach in which blade forces are represented by employing a line with distributed loads. This model was tested while giving promising results in comparison to the MEXICO experimental data [44]. The actuator line approach was expanded to the actuator surface method (Shen et al.) [45] by representing a wind turbine blade as a planar surface. Actuator type models provided a better understanding of the wake dynamics. Solving the Navier-Stokes equations in this model takes more time than using the BEM model. Also, loads on the blade that were calculated using blade element theory and actuator-type model are not as accurate as the aerodynamic data obtained from the BEM model.

\subsubsection{Vortex Model}

Another model that is used widely in the aerodynamic analysis of wind turbines is the Vortex model. This model calculates the induced velocity by using the Biot-Savart law from the bound vortices on the blade as well as the trailing vortices in the wake, which are 
represented using lifting surface, lifting line, or vortex lattice methods. Vortex methods are mainly divided into three different types: 1) Prescribed wake model, 2) Rigid wake model, and 3) Free wake model. The rigid wake model has a significant weakness, since it does not calculate the expansion of the wake, resulting in not so accurate blade load calculations [46]. This weakness was solved by introducing the prescribed wake model, which uses experimental data or other numerical results to find the wake location [47] [48] [49]. Free wake model calculates the motion of vortices directly by using the effects of other factors on the wake vortex system with consideration of blade effects at different time steps [50] [51] [52] [45]. The free wake model can better predict the aerodynamic behavior of a complex flow field, like the one generated by wind turbines, but using this model requires more computational time and main memory. Vortex model is known to be more accurate than BEM.

BEM and Vortex methods need reliable airfoil aerodynamic data as an input to be able to calculate the results accurately; this data are acquired using wind tunnel experiments for a wide range and resolution of Reynolds numbers and angles of attacks. During the simulation and in some extreme conditions, turbine's blade may encounter high Reynolds number and angles of attack, so an aerodynamic airfoil data with a wide range of angle of attacks and Reynolds numbers are required for an accurate and robust calculation. Additional data outside the test range is obtained by accepting that the aerodynamic data can be extrapolated in a situation which flow on one side of the airfoil is completely separated with high angles of attack. This model has some weaknesses in the calculation of the pitching moment coefficient [53]. Later, Lindenburg developed some semi-empirical relations by analyzing massive test data of different airfoil geometries and achieved good 
agreement with available experimental data [54]. There is also another option for achieving airfoil aerodynamic coefficients, which is using numerical simulations. XFOIL is a tool that is extensively used for airfoil design and aerodynamic evaluation, integrating the viscous/inviscid analysis methods and generating relatively accurate results before stall. Viscous method is established using integral boundary layer equations that cannot be used in flow separation; hence stall properties are deemed unreliable. To address this issue, some numerical methods like DES (Detached Eddy Simulation) [55] [56] and LES (Large-Eddy Simulation) [57] [58] were proposed, while giving excellent results in some specific conditions.

\subsection{Aeroacoustics}

Aeroacoustics is the study of sound generated by airflow. Aeroacoustics gained attention in the 1950s when noise produced by jet engines became a concern. Lighthill [59] was the first person who suggested an aeroacoustics analogy that transformed the compressible Navier-Stokes equations into an inhomogeneous wave equation; his equation defines a wave equation in terms of fluctuating densities. Although Lighthill's equation has no assumption for the boundaries, using his wave equation with proper boundary conditions can provide accurate results. Green's function for free field radiation was used by Lighthill for defining the solution of the wave equation. Scattering absorption resonators and bodies in the neighborhood of the sources as well as reflection by solid boundaries were not considered by Lighthill.

Later, Curle expanded Lighthill's integral representation to consider solid body influence [60]. In most of the technical applications, like helicopter rotors or industrial fans and turbines, moving solid surfaces have an impact on the noise generation. There are some 
other methodologies that can predict the noise generated by rotating blades. These methods follow a solid physical and mathematical basis using Lighthill acoustic analogy and Kirchhoff formulations for subsonic and supersonic moving surfaces.

Kirchhoff formulation can be considered as an inhomogeneous wave equation considering the distribution of acoustic sources on a surface that surrounds all physical sources [61]. Kirchhoff's surface needs to be placed in a linear flow region, which is not well defined. Kirchhoff formulation does not come up with reliable results in situations that surface is not placed carefully or when wake passes through the surface [62] .

In 1969, Ffowcs, Williams, and Hawkings developed a new Kirchhoff formulation for moving surfaces, which became associated with their names. They used a strong technique of the generalized function theory for the development of their equation. FW-H equation is precisely a rearrangement of two equations, "Navier-Stokes and continuity" in form of an inhomogeneous wave equation with two surface source terms which are monopole and dipole, as well as a volume source term which was called quadrupole [61]. In the FW-H equation, three source distributions are being differentiated, which are the thickness and loading surface sources, in addition to the quadrupole volume source. Noise also would be captured by two sources, the one that was produced by the displacement of fluid would be captured by the thickness source, and the noise produced by unsteady force distribution over the body surface would be captured by the loading source [62].

There are three different problem types when motion of the observer and fluid speed are considered. The first one is called fly over, which can be used when sound is seized by a stationary observer in a stationary ambient medium. Second type of problem is called moving observer, which can be defined when observer is moving, for example, when a 
microphone is placed on the fuselage of a helicopter or an aircraft. The last one is called wind tunnel and can be used when sound is received by a stationary observer in a moving medium, like sound calculation in a wind tunnel.

A problem can be transformed from one type into an equivalent problem of a different type if Galilean transformation is used, for instance a wind turbine problem in which the medium has a velocity of $U_{0}$ can be transformed into a moving observer in which source and observer are moving with a velocity of $U_{0}$ while the medium is stationary. The main purpose of such transformations is making the problem simpler and lower the overall computational costs.

Although the Ffowcs Williams-Hawkings (FW-H) equation and its related formulations like Farassat's Formulation 1A and 1 have low computational costs, they are also robust. They could be used successfully in a wide range of fly-over and movingobserver problems. Formulations $1 \mathrm{~A}$ and 1 do not consider presence of a mean flow for wind tunnel problems. One solution to this issue is to change a given wind tunnel problem into a moving observer problem in which an observer is considered to move at a constant speed in a quiescent environment. Another solution is having an explicit consideration of a mean flow by solving a convective wave equation.

Another approach suggested by Najafi-Yazdi et al., which is an upgraded version of Formulation 1A. This method considers the contribution of a uniformly moving medium; this approach is called Formulation 1C [63]. VAWT needs wind for its operation; hence associated convective impacts should be considered. In the problems where the medium's velocity is zero, Formulation $1 \mathrm{C}$ is equal to the Formulation 1A [63]. There are also other tools to calculate the acoustics of moving sources in a moving medium. They are 
successfully utilized; an example of that can be Wells and Han's approach for the propeller noise. However, their strategy could not be well represented as Najafi Yazdi's work.

Another solution of the FW-H equation is suggested by Casper and Farassat, which is called Formulation 1B that calculates the loading noise coming from an unsteady surface pressure distribution on a thin airfoil in the time domain. This approach can predict the broadband noise of an airfoil interacting with homogeneous turbulence which can predict the trailing edge noise [64]. Five methods of airfoil self-noise are introduced by Brooks et al., which used the interactions between an airfoil blade and the turbulence made in its own boundary layer and near wake. These methods are: 1) Separation-stall noise, 2) TEbluntness vortex-shedding noise, 3) Tip vortex formation noise, 4) Turbulent-boundarylayer-TE noise, and 5) Laminar-boundary-layer-vortex-shedding noise [65]. These models are missing certain acoustic sources that affect accuracy of broadband noise simulations. [65]

There has been not much analysis of aeroacoustics performed for VAWTs; Kelly et al. [66] have done the most important study on this topic. This study includes the aeroacoustics analysis of the 17-m Troposkien VAWT built by Alcoa and the 24-m NRC/ DAF Troposkien VAWT. Another experimental work done by Pearson includes semiempirical modeling on a VAWT straight-bladed prototype [67] [68].

\subsection{Arrangement of Thesis}

In the first chapter, a history of wind energy was presented along with a brief discussion concerning different types of wind turbines as well as existing methods to calculate and predict the aerodynamics and aeroacoustics of VAWTs. In the second chapter, the aerodynamic analysis methodology used in this thesis is described and different 
aerodynamic quantities are calculated. The methodology is verified and validated against available experimental data. In the third chapter, aeroacoustics methodology which used in this study is described and then, in the fourth chapter, aerodynamic and aeroacoustic results are presented. Fifth chapter includes conclusions and suggestions for future work. 


\section{Chapter 2:}

\subsection{Aerodynamic Analysis}

Aerodynamic modelling of VAWTs has been always challenging due to the mutual influence between the moving blades and their wake. As mentioned before, aerodynamic data is required to determine the aeroacoustic behavior of a turbine.

In the previous chapter, different aerodynamic models were described. Although Computational Fluid Dynamics (CFD) modelling can be used successfully in the aerodynamic prediction of wind turbines, some shortcomings still exist, i.e., relatively high computational costs (CPU time and main memory usage), cumbersome meshing (requiring both fixed and rotational computational domains with interpolation errors between them), and dissipative nature of turbulence models (affecting the wake dissipation), all factors combined, affect the overall simulation.

Another approach is adopted here. Aerodynamic modelling of a turbine using a coupled panel method with a vortex wake model can become an advantageous alternative [69] [70], providing precise aerodynamic data for wind turbines and requiring less computational time and expense compared to the CFD approach [71].

Through collaboration with the University of Athens, an in-house code named GENeral Unsteady Vortex Particle (GENUVP, developed by Voutsinas and co-workers [72] [73] [74] [75]) which uses a panel method coupled with a vortex wake model will be used in the present work. In the following sections, GENUVP code structure and its main theoretical aspects are described. 


\subsection{GENUVP Concept}

Helmholtz decomposition which is also known as the "vorticity transport theorem" represents the fundamental aspect of the GENUVP code. Utilizing this decomposition, the surrounding flow field over a VAWT can be divided into rotational and irrotational parts, due to the wake released by lifting bodies and the presence of multiple bodies respectively.

Consider Equation (2.1) as the velocity around a VAWT. This equation is a function of position and time $(\vec{x}, t)$ and $\mathrm{D}$ is defined as the flow field domain.

$$
\vec{u}(\vec{x}, t), \vec{x} \in D, t \geq 0 .
$$

Employing Helmholtz decomposition, the flow field around a turbine can be described as:

$$
\vec{u}(\vec{x}, t)=\vec{u}_{\text {ext }}(\vec{x}, t)+\vec{u}_{\text {solid }}(\vec{x}, t)+\vec{u}_{\text {near-wake }}(\vec{x}, t)+\vec{u}_{\text {far-wake }}(\vec{x}, t),
$$

where $\vec{u}_{\text {ext }}$ has a specific quantity and stands for the external velocity, $\vec{u}_{\text {solid }}$ can be defined as field velocity due to the presence of solid bodies. $\vec{u}_{\text {near-wake }}$ and $\vec{u}_{\text {far-wake }}$ are near-wake and far-wake velocities because of being in a distance of $\mathrm{D}$, from near-wake and far-wake. $\vec{u}_{\text {solid }}$ and $\vec{u}_{\text {near-wake }}$ can be taken as singularity distributions over the surface of solid bodies and near wake, respectively, as the panel method is employed. Using the Biot-Savart law, $\vec{u}_{\text {far-wake }}$ can be calculated as:

$$
\vec{u}_{\text {far-wake }}(\vec{x}, t)=\int_{D_{\omega}(t)} \frac{\vec{\omega}\left(\vec{x}_{0}, t\right) \times\left(\vec{x}-\vec{x}_{0}\right)}{4 \pi\left|\vec{x}-\vec{x}_{0}\right|^{3}} \mathrm{dD} .
$$

Vortex methods, which define the vorticity in the flow field, can be used to evaluate Equation (2.3). Hence, a coupled panel method and vortex model with proper coupling conditions can be used to compute Equation (2.2). 


\subsection{Panel Method $\left(\vec{u}_{\text {solid }}\right.$ and $\left.\overrightarrow{\boldsymbol{u}}_{\text {near-wake }}\right)$}

Following Hess, a panel method is used in GENUVP code to compute the induced velocity in the flow field because of the presence of solid bodies [76] [77]. Continuity equation can be considered as the Laplace equation for a body with a surface $\mathrm{S}$ due to existence of an incompressible and inviscid flow.

$$
\nabla^{2} \phi=0
$$

In Equation (2.4), $\phi$ is defined as the scalar velocity potential. Two conditions could be considered for the solution of the potential: the first one is the condition of non-penetration of solid boundaries (2.5) and the second one is the condition of regularity (2.6).

$$
\begin{gathered}
\left.\nabla \phi \cdot \vec{n}\right|_{s}=\left.\frac{\partial \phi}{\partial n}\right|_{S}=\left(\vec{u}_{\text {ext }} \cdot \vec{n}-F\right)_{S} \\
|\nabla \phi|_{\infty} \rightarrow 0 .
\end{gathered}
$$

In the first Equation (2.5), $\vec{n}$ can be defined as the local outward-normal unit vector on the surface $\mathrm{S}$, and $\mathrm{F}$ can be defined as the local normal velocity because of the body's motion and induced velocity of far-wake. Solutions to Equation (2.4) may be structured in terms of source and dipole distributions over the surfaces of the bodies in the flow field using Green's identity. The potential because of the presence of a non-lifting body can be obtained by describing continuous source distribution over the surface of the body. Consider $\sigma$ as a local source intensity distribution on the body, then the potential generated at the point $\vec{P}$ because of the existence of the body in the flow can be defined as:

$$
\phi(\vec{P})=\int_{S} \sigma\left(\frac{1}{r}\right) d S
$$

For a lifting body, the potential can be obtained from defining a dipole distribution (thin body) or alternatively from source and dipole distributions (thick body). In order to 
generate circulation around the body and consequently creating a net lift force, a dipole distribution is needed on the lifting body. Also, according to the Kelvin's theorem, to meet the conservation condition of circulation, dipole sheets are trailed downstream of the lifting body to generate the wake surfaces. By considering $\mu$ as the local dipole intensity distribution on a body or wake surface, the potential generated at a given point $\vec{P}$ because of the dipole distribution on the lifting body or wake surface can be defined as:

$$
\phi(\vec{P})=\int_{s} \mu \vec{n} \cdot \nabla\left(\frac{1}{r}\right) d S .
$$

Also, the potential due to the existence of solid bodies, at a given point $\vec{P}$ in the flow field can be defined as:

$$
\begin{gathered}
\phi(\vec{P})=-\frac{1}{4 \pi} \int_{\text {lifting }} \mu \vec{n} . \nabla\left(\frac{1}{r}\right) d S-\frac{1}{4 \pi} \int_{\substack{\text { Non } \\
\text { lifting }}} \sigma\left(\frac{1}{r}\right) d S \\
-\frac{1}{4 \pi} \int_{\text {Wake }} \mu \vec{n} . \nabla\left(\frac{1}{r}\right) d S .
\end{gathered}
$$

It is worth mentioning that Equation (2.9) should be used when $\vec{P}$ is on one of the surfaces. As Equations (2.7) and (2.8) meet the regularity condition of Equation (2.6), nonpenetration boundary is the only condition that needs to be considered. Combination of Equations (2.5) and (2.9) can satisfy the non-penetration boundary condition:

$$
\begin{aligned}
\left\{-\frac{1}{4 \pi} \int_{\text {lifting }} \mu \nabla\left[\frac{\partial}{\partial n}\left(\frac{1}{r}\right)\right] d S-\frac{1}{4 \pi} \int_{\begin{array}{c}
\text { non- } \\
\text { lifting }
\end{array}} \sigma \nabla\left(\frac{1}{r}\right) d S\right. \\
\left.-\frac{1}{4 \pi} \int_{\text {wake }} \mu \nabla\left[\frac{\partial}{\partial n}\left(\frac{1}{r}\right)\right] d S\right\} \cdot \vec{n}=\vec{u}_{\text {ext }} \cdot \vec{n}-F .
\end{aligned}
$$


Equation (2.10) can be used for all body surfaces and it is the main equation for the panel method that is used in the GENUVP code.

Equation (2.10) can be estimated by a system of linear equations if body surfaces and wakes are divided into the panel elements. In each panel, there is either a source intensity $(\sigma)$ or a dipole intensity $(\mu)$. Notice that the existence of a source or dipole intensities depend on whether there is a non-lifting surface $(\sigma)$ or a lifting surface or a wake surface $(\mu)$, respectively. Source and dipole intensities can be taken out of integral in Equation (2.10). Residual integrals are based on the configuration geometry and discretization.

By calculating integrals at the control point of elements, constant matrices of the influence coefficients can be defined as:

$$
\begin{gathered}
{\left[C_{i j}^{\text {lifting }}\right]\left\{\mu_{j}\right\}+\left[C_{i k}^{\text {near-wake }}\right]\left\{\mu_{k}\right\}+\left[C^{\text {non-lifting }}{ }_{i l}\right]\left\{\sigma_{l}\right\}=\left\{\vec{u}_{\text {ext }} \cdot \vec{n}_{i}-F_{i}\right\}} \\
i=1,\left(N_{\text {lifting }}+N_{\text {non-lifting }}\right), \\
j=1, N_{\text {lifting }} \\
k=1, N_{\text {near-Wake }} \\
l=1, N_{\text {non-lifting. }}
\end{gathered}
$$

Equation (2.11) can be described as the linear system approximation of Equation (2.10). Total number of panels on the lifting, near wake, and non-lifting surfaces are presented as $N_{\text {lifting }}, N_{\text {near-Wake }}$, and $N_{\text {non-Lifting }}$.

Specific physical conditions should be utilized in the near-wake to achieve a unique solution for the system [74]. A zero pressure jump Kutta condition is caused when wake strip elements' dipole intensity has the same value as the adjacent emitting elements on lifting bodies' tip and trailing edges. Then, flow speed at the emission edges can be used to estimate the near wake geometry. 
Scalar velocity potential can be computed at any required point in flow field using a discretized version of Equation (2.9). This computation is valid only when dipole and source distributions are calculated using Equation (2.11). Considering the potential, an estimation of the velocity field can be obtained using Equation (2.2):

$$
\vec{u}_{\text {Panel }}=\vec{u}_{\text {solid }}+\vec{u}_{\text {Near-Wake }}=(\nabla \phi) .
$$

Using the unsteady Bernoulli equation, pressure distribution over a solid body can be calculated, then potential load on lifting bodies can be determined from pressure distribution. The unsteady Bernoulli equation is determined as follows:

$$
\dot{C}_{p}=1-\frac{u^{2}}{u_{r e f}^{2}}-\frac{2}{u_{r e f}^{2}} \frac{\partial \phi}{\partial t} .
$$

\subsection{Vortex Particle Methods - $\overrightarrow{\boldsymbol{u}}_{\text {far-Wake }}$}

Using the vortex particle method, wake structures released from lifting bodies can be traced within the flow-field boundaries. Different models can be used to define the wake structure, for example, vortex segment (filament) elements, constant strength vorticity contours or vortex blobs. After that, the wake-induced velocity at a particular point can be calculated by using the Biot-Savart law, or Equation (2.3).

There are two main vortex wake models, i.e., fixed or free wake models. The fixed wake model uses analytical relations or experimental data to determine the location and shape of the wake. On the other hand, the free wake model uses numerical integration of vorticity transport equations. The free wake model needs more computational time and cost as discretization is required for tracking the wake structure, causing a high number of calculations. It should be noted that the free wake model produces more accurate results 
than the fixed wake model. Some suitable approximation can be used to decrease the computational cost of the free wake model.

The GENUVP code uses the vortex blob method for the far-wake modelling [78]. Majda and Beala substantiate that vortex blobs method is an accurate, stable, automatically adaptive, and convergent vortex method. The vortex blob method defines the wake using a cloud of vortex particles in which each particle has its own position, intensity, and velocity vector quantities [79]. Also, regrouping of wake vortices (more relevant in mixing layers) are not necessary in the current simulations.

According to Equation (2.3), $D_{\omega}(t)$ is divided into volume components $\left(D_{\omega, j}(t), j \in J(t)\right)$. Each of these volume components has an assigned vortex particle. Vorticity and location of the $\mathrm{j}$-th vortex particle are defined as $\vec{\Omega}_{j}(t)$ and $\vec{Z}_{j}(t)$, respectively. The vorticity can be calculated using the following equation:

$$
\vec{\Omega}_{j}(t)=\int_{D_{\omega, j}} \vec{\omega}(\vec{x}, t) d D
$$

Where,

$$
\begin{gathered}
\vec{\omega}(\vec{x}, t)=\sum_{j} \vec{\Omega}_{j}(t) \delta\left(\vec{x}-\vec{Z}_{j}(t)\right), \\
\vec{\Omega}_{j}(t) \times \vec{Z}_{j}(t)=\int_{D_{\omega, j}} \vec{\omega}(\vec{x}, t) \times \vec{x} d D
\end{gathered}
$$

By utilizing the vortex particle method, the Biot-Savart law for the far-wake induced velocity is defined as:

$$
\vec{u}_{f a r-w a k e}(\vec{x}, t)=\sum_{j} \frac{\vec{\Omega}_{j}(t) \times\left(\vec{x}-\vec{Z}_{j}(t)\right)}{4 \pi\left|\vec{x}-\vec{Z}_{j}(t)\right|^{3}} .
$$


This equation has a high singularity, hence, Beale and Majda [80] suggested an approximation which is stated as follows:

$$
\begin{gathered}
\vec{u}_{\text {far-wake }}(\vec{x}, t)=\sum_{j} \frac{\vec{\Omega}_{j}(t) \times \vec{R}_{j}}{4 \pi R_{j}^{3}} f_{\varepsilon}\left(R_{j}\right) \\
\vec{R}_{j}=\vec{x}-\vec{Z}_{j}(t) \\
f_{\varepsilon}\left(R_{j}\right)=1-e^{\left(R_{j} / \varepsilon\right)^{3}}
\end{gathered}
$$

where $\varepsilon$ is the vortex particle cut-off length. The vortex blob convection is executed in a Lagrangian form, which is described below:

$$
\begin{gathered}
\frac{d \vec{Z}_{j}}{d t}=\vec{u}\left(\vec{Z}_{j}, t\right) \\
\frac{d \vec{\Omega}_{j}}{d t}=\left(\vec{\Omega}_{j} \nabla\right) \vec{u}\left(\vec{Z}_{j}, t\right)=\overleftrightarrow{D} \cdot \vec{\Omega}_{j}
\end{gathered}
$$

where $\stackrel{\leftrightarrow}{ }$ is defined as the deformation tensor.

\subsection{Near-Far Wake Coupling Conditions}

Near wake strip elements are converted to vortex particles and then become a new part of far-wake, after each time step of the panel method calculation is finished. Near-wake strip elements become a part of the far-wake by applying integration on the vorticity of each near-wake dipole component to generate a vortex particle. New vortex particles then turn into far-wake, and this happens before the next time step. Figure 7 demonstrates the vortex blob formation process. 

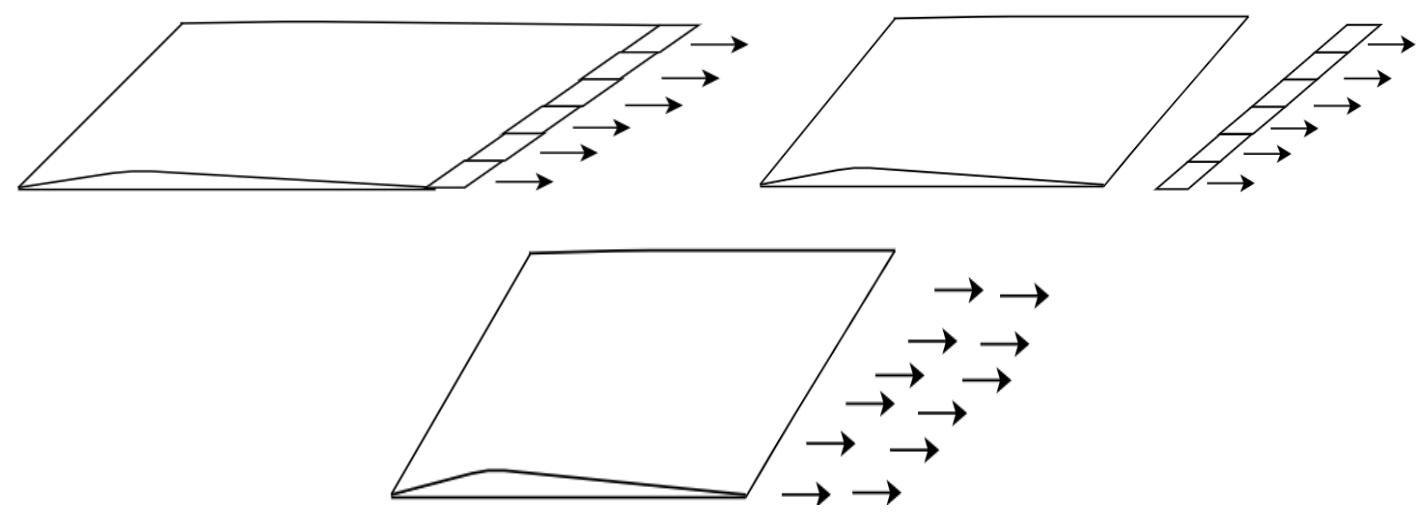

Figure 7 Vortex Particles Formation from a Trailing-Edge Wake Strip.

\subsection{Efficiency improvements in GENUVP}

In the GENUVP code, sub-grid and particle mesh approximations are applied to increase the code's efficiency and to reduce its computational costs [74] [81]. The main reason for using sub-grid approximations in this code was to reduce the calculation in the panel method. Hess stated that using exact integral evaluations are required in situations that the distance between an evaluation control point and the inducing panel is small [76]. Indeed, when the gap between the evaluation point and the inducing panel is more than 4 times of the panel's maximum diagonal, then the integral calculation can turn into a point calculation. We can anticipate the error to be minor when the distant panel, in which the integrals are evaluated on, clustered into larger groups [82]. Figure 8 shows the different levels of refinement [81]. It is worth mentioning that computations begin at coarse paneling and then, based on the space between the panel center and the evaluation point, calculations would continue either with the integral evaluation over a large panel or go to a more refined panel. 

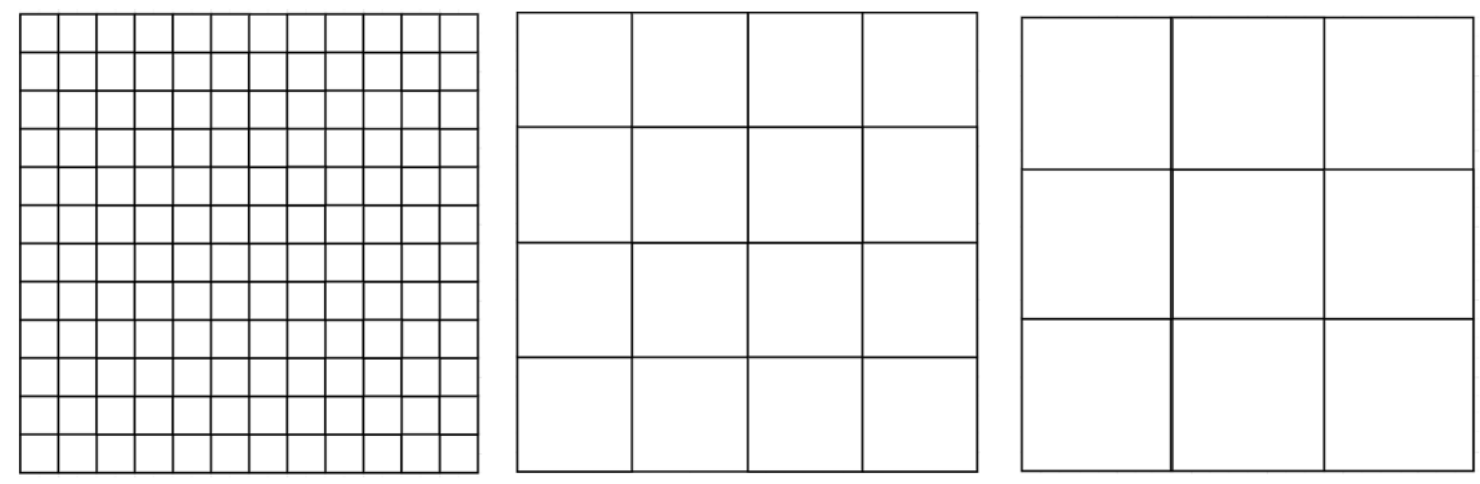

1) Different Paneling Levels

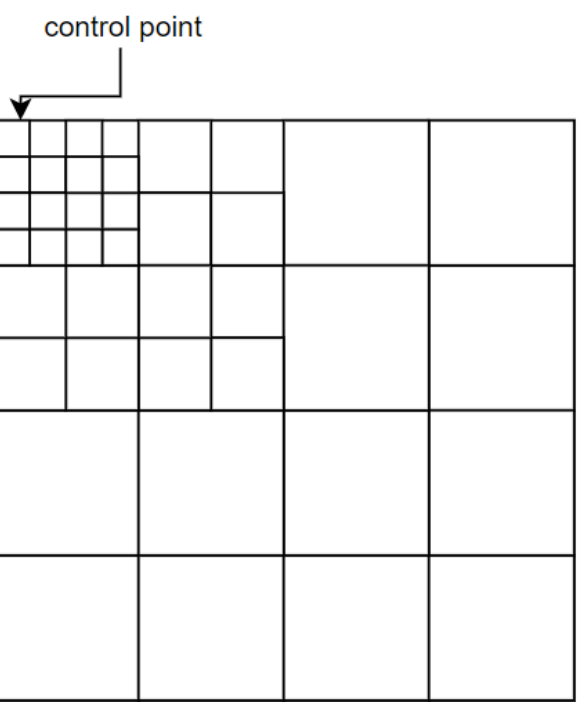

2) Activation of Different Levels

Figure 8 Grid Refinement Diagram for Different Levels Used for Subgrid Approximation.

A surface panel $\mathrm{S}$ with $\mathrm{n}$ panels at the highest discretization level with an unknown singularity of $\mathrm{X}$ is considered. Collective contribution of $\mathrm{n}$ panels can be calculated using the following equation:

$$
\mathrm{I}=\sum_{i=1}^{n} I_{1} \frac{S_{i}}{S} X_{i} .
$$

In the above equation, $I_{1}$ is the integral value calculated over the surface $S$ and $S_{i}$ is the surface area of the i-th high-level panel. 
In the conventional vortex approach, velocity and the deformation in every blob position using the Biot-Savart Law is calculated. In the aforementioned method, $N^{2}$ pointto-point calculations for a complete time step of $\mathrm{N}$ vortex blobs is needed. $\mathrm{N}$ also increases as the simulation goes on, causing a higher computational time and expense. To remedy this issue, particle-mesh (PM) techniques can be used to decrease the $N^{2}$ point-to point calculations to $\mathrm{N} \cdot \log (\mathrm{N})$ calculations in each time step [83].

When the number of blobs is high, $\vec{u}$ and its spatial derivatives (deformation tensor $\overleftrightarrow{D}$ ) are calculated at the nodes of a Cartesian grid having $D_{\omega}(t)$. To calculate the value of $\vec{u}$ and $\overleftrightarrow{D}$ at the precise location of the blobs, local interpolation is performed. $\vec{u}_{f a r-w a k e}$ 's vector potential $(\vec{A})$ can be defined as:

$$
\nabla \times \vec{u}_{\text {far-wake }}=\vec{A}
$$

Using the Fourier method, the corresponding Poisson equation is obtained:

$$
\begin{gathered}
\nabla^{2} \vec{A}=-\vec{\omega}, \\
\text { in } D_{\omega}(t) .
\end{gathered}
$$

In each PM (Particle Mesh) calculation, three stages are followed. They are projection stage, solution stage and interpolation stage. In the first stage, "Projection" Vorticity would be calculated on a cartesian mesh which contain all vortex blobs. Calculations involve projection of the intensity vortex blobs that are placed within a cell of mesh on the vertices of the cell. In the solution stage, a discretization of Equation (2.23) would be done by using standard central differences. Three heptadiagonal linear systems are achieved then, $\vec{A}$ values at the grid boundary nodes, are calculated using point to point Biot-Savart calculations. After that, linear systems are solved for nodal values of the vector potential $\vec{A}$ 
using Fourier method. Standard central differences can be utilized to compute the speed and deformation in different nodes of grid when vector potential's nodal values are found. In the end, speed and vorticity deformation in each vortex blob can be calculated using interpolation between nodal values of the adjacent grid nodes.

As the grid cell size limits the PM method's accuracy, local corrections are suggested by Anderson to reduce the PM method error [84]. Based on experience, corrected PM method is not reliable and accurate to be used for the important parts of geometry. In the GENUVP code, a hybrid method is used so that, in the vicinity of the solid boundaries Biot-Savart law is applied, and for the other parts PM (Particle Mesh) calculations is used. 


\section{GENUVP Code}

This code is written in Fortran 90 and parallelized. A diagram of GENUVP operation is shown in Figure 9. This code has three main parts: initialization, potential calculations, and vortex calculations.

In the first "initialization" part, the problem is transferred to the code by input files, which contain ambient condition, blade geometry, pitch control input, etc. In the next step ("potential"), Kutta condition and non-penetration boundary conditions are applied and governing equations are solved to generate the output data for that particular time step. In the final step ("vortex calculations"), new wake elements are generated from near-wake panel strips. The particle wake is transferred downstream, before the initialization of the next "potential" calculations.

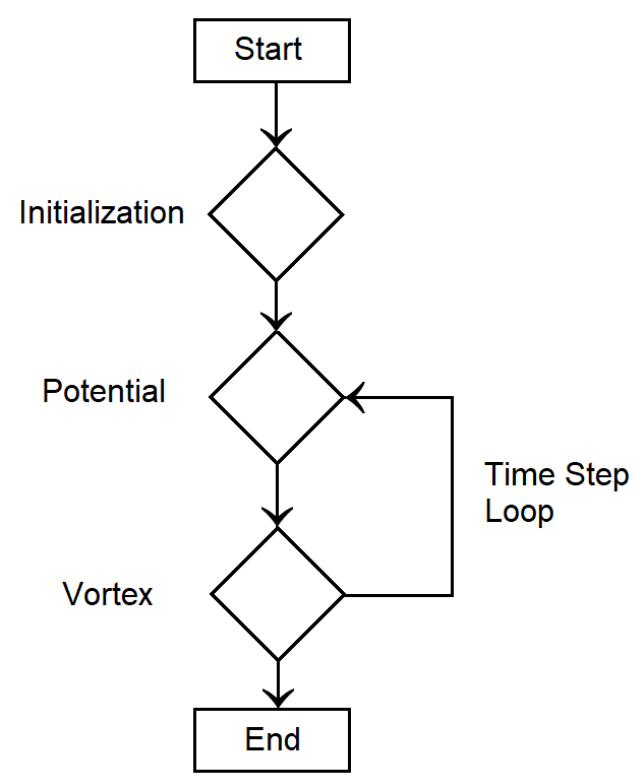

Figure 9 Top Level Block Diagram of GENUVP. 


\subsection{Troposkien geometry}

Troposkien geometry is achieved from balancing forces on a uniform skipping rope that rotates vertically with a persistent velocity. A general equation for the Troposkien geometry is suggested by Blackwell and Reis that is provided as follows [85]:

$$
\frac{z}{a}=1-\frac{F(\phi, k)}{F\left(\frac{\pi}{2}, k\right)}
$$

where $z$ is the vertical position, $a$ is the half height of the blade and $F$ is the complete elliptical integral of the first kind with the parameter $k$ described as:

$$
\begin{gathered}
F(\phi, k)=\int_{0}^{\phi} \frac{d \theta}{\sqrt{1-k^{2} \sin ^{2} \theta}}, \\
\phi=\sin ^{-1}\left[\frac{1}{\beta}\left(\frac{\dot{r}}{a}\right)\right]
\end{gathered}
$$

where $r$ is defined as radius of the blade at a specific height, $b$ is the radius at the equator of blade, and $\beta$ is the diameter to height ratio. $\beta$ is related to $k$ using the following equation

$$
\beta=\frac{2 k}{\left(1-k^{2}\right) F\left(\frac{\pi}{2}, k\right)} .
$$




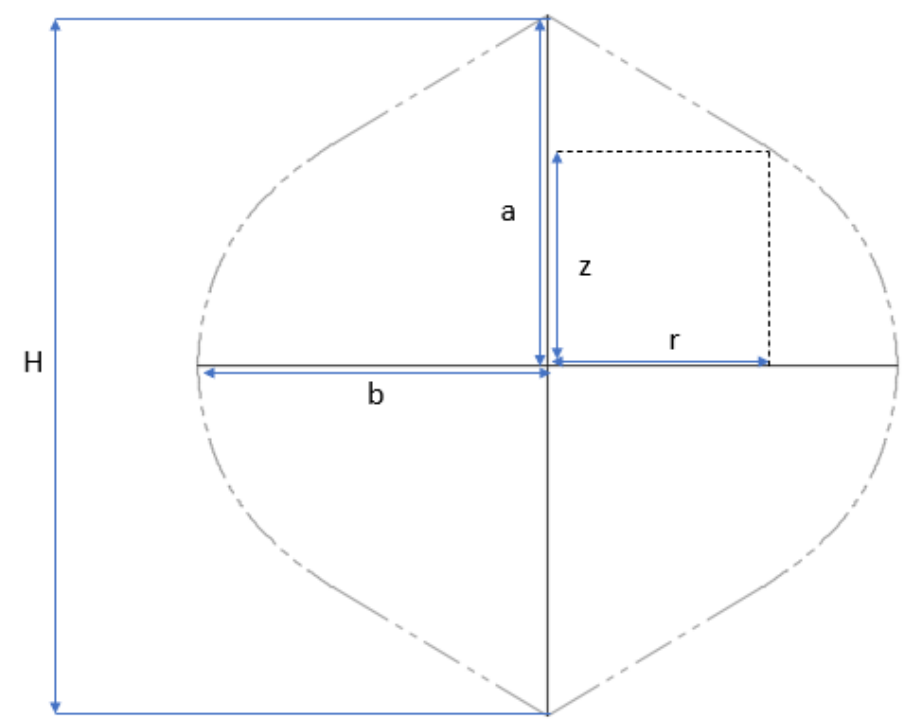

\section{Figure 10 Conventional Troposkien Turbine with Some Geometric Parameters.}

Integrals can be calculated numerically using iterative determination of $k$ as a function of $\beta$. Troposkien blade length, $S$, and swept area, $A_{s}$, can be calculated using the following equations:

$$
\begin{gathered}
\frac{\dot{S}}{2 a}=\frac{2}{1-k^{2}} \frac{E\left(\frac{\pi}{2}, k\right)}{F\left(\frac{\pi}{2}, k\right)}-1, \\
\frac{A_{s}}{4 a^{2}}=\frac{\ln \left(\frac{1+k}{1-k}\right)}{\left(1-k^{2}\right) F^{2}\left(\frac{\pi}{2}, k\right)}
\end{gathered}
$$

$E\left(\frac{\pi}{2}, k\right)$ is the complete second kind elliptical integral with the parameter $k$ given by:

$$
E(\phi, k)=\int_{0}^{\phi} \sqrt{1-k^{2} \sin ^{2} \theta} d \theta
$$

Construction of a pure Troposkien blade is not cost effective for a manufacturer as each point on the blade has its own local curvature, on the other hand, following the shape of a 
Troposkien blade is important since the bending stress along the blade is minimal. An alternative approach is to model and create a straight/arc blade, that has a circular part at the middle with two connected straight parts. This model can approximate the Troposkien configuration.

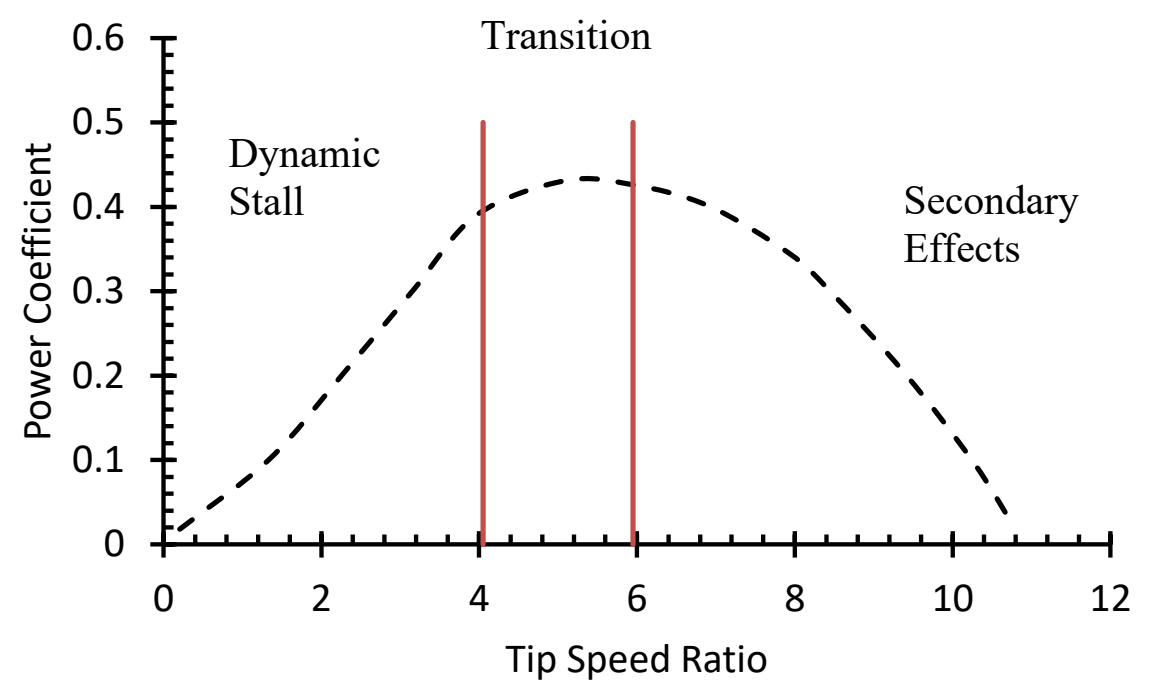

Figure 11 Darrieus Turbine Performance Characteristics [5].

Figure 11 shows a typical curve of power coefficient $C_{p}$ as function of the TSR (Tip Speed Ration) for a VAWT. According to the figure, three different regions exist during operation. In the low TSR (TSR $<4)$, the wind turbine blades experience dynamic stall and at high TSR (TSR > 6), secondary effects (due to presence of struts, rotating tower, and blade wake interactions) dominate the flow. Dynamic stall and secondary effects negatively affect the performance of the turbine. In the transition zone $(4<\mathrm{TSR}<6)$, dynamic stall and secondary effects do not hinder the overall efficiency of the turbine.

It is important to control the operation condition of turbine e.g., rotational speed, to make sure that turbine is operating in its optimum condition and generating the highest 
output power. In HAWT, pitching control and nacelle rotation can be used while for the VAWT, blade twisting and using two generators can be considered.

For a higher accuracy of the results in the dynamic stall zone, ONERA [86] dynamic stall model is utilized in the current study for the lift, drag and moment coefficients calculations.

\subsection{Aerodynamic Validation}

To make sure that the aerodynamic model adopted in this study is dependable and working properly, two sets of studies are considered. Numerical aerodynamic results in this study is being validated against the experimental results done by Sandia Laboratories, which is a standard and dependable source that provided aerodynamic analysis for different wind turbine sizes (2-m,5-m,17-m,34-m) and in various operation conditions. In the first study, a 2-m Sandia VAWT is modeled in GENUVP and aerodynamic behavior is predicted. Results are then examined against available experimental data. In the second scenario same study is performed on a 17-m Sandia VAWT. The characteristics of these two turbines are defined in the following table: 
Table 1 Geometric Characteristics of 2-m and 17-m Sandia VAWT

\begin{tabular}{|c|c|c|}
\hline Parameter & 2-m Sandia VAWT & 17-m Sandia VAWT \\
\hline Number of Blades & 2 & 2 \\
\hline Turbine Radius $(\mathrm{m})$ & 1 & 8.36 \\
\hline Turbine Height $(\mathrm{m})$ & 2 & 17 \\
\hline Blade Height $(\mathrm{m})$ & 2 & 17 \\
\hline Blade Length $(\mathrm{m})$ & 2.97 & 24.71 \\
\hline Swept Area $\left(\mathrm{m}^{2}\right)$ & 2.59 & 187.1 \\
\hline Chord Length $(\mathrm{m})$ & 0.08815 & 0.53 \\
\hline Solidity & 0.202 & 0.14 \\
\hline Airfoil & NACA0012 & NACA0015 \\
\hline
\end{tabular}

The first turbine is the 2-m Sandia VAWT [87] and the second one is the 17-m Sandia VAWT [88]. These two turbines were designed and built by Sandia Laboratories and their aerodynamic behavior is studied using experimental analysis. Note that the aerodynamic behavior prediction provided in this study is exactly same as the experimental analysis done by Sandia, so Table 1 is valid for both experimental and numerical analysis.

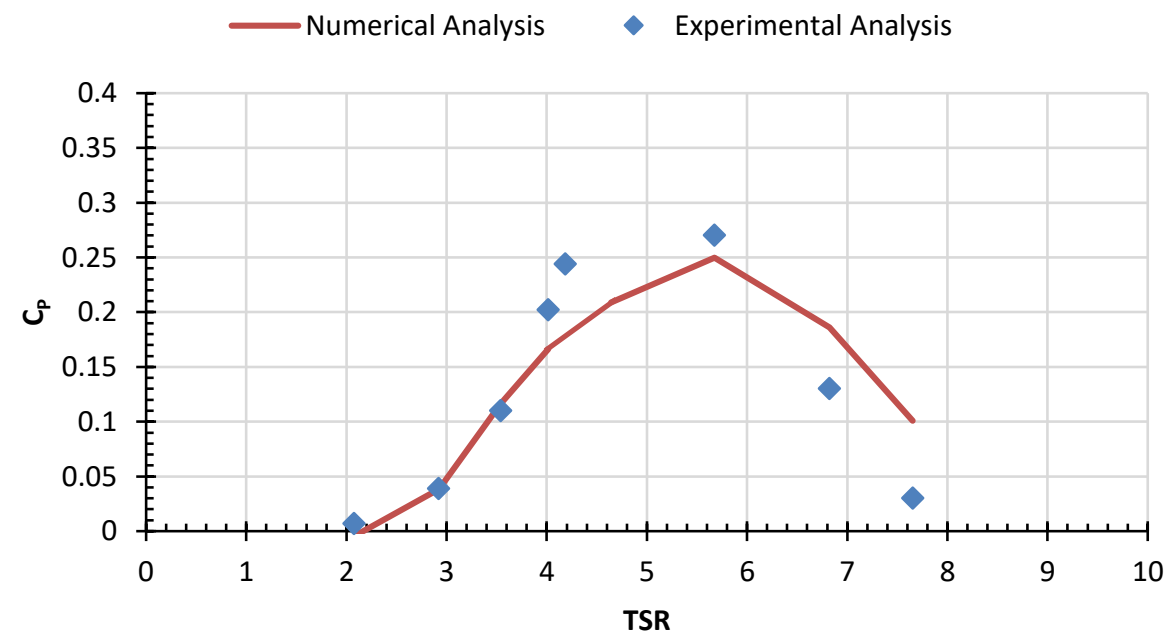

Figure 12 Performance Comparison of the SANDIA 2-m VAWT (27.9 rad/s). 
Figure 12 shows the aerodynamic performance analysis for the Sandia 2-m VAWT. The blue diamond symbols represent the experimental data by Sandia and the solid red line depicts current numerical simulation results using GENUVP at the same boundary conditions (additional convergence analysis will be discussed later). Notice that the turbine starts with low power coefficients at low TSRs (or high wind speed when the rotation is kept constant), reaches a peak around TSR $=5.6$, then the power coefficient is further reduced when the TSR is increased (lower wind speeds). Overall, there is a relatively good agreement between the numerical results and the experimental data, with slight underprediction in the transitional region and overprediction when secondary effects become important at higher TSRs.

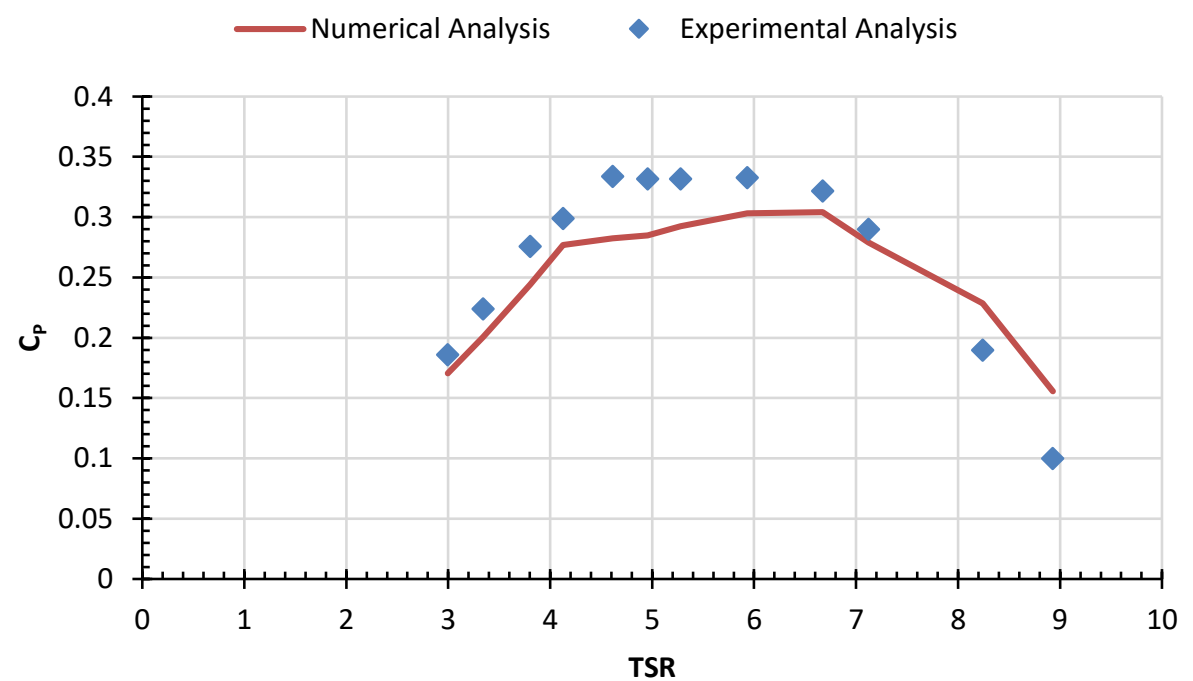

Figure 13 Performance Comparison of the Sandia 17-m VAWT (5.5 rad/s). 
Figure 13 shows similar comparison between current simulation results and the experimental data for the Sandia 17-m VAWT. This turbine starts with a power coefficient of 0.19 at the TSR $=3.0$ and reaches its peak with a value of 0.33 . Simulation and experimental results are also relatively close to each other for the 17-m VAWT.

The following figure demonstrates the wake generated from the turbine after five complete cycles. This simulation belongs to a 2-m Sandia conventional design with rotational velocity of $27.9 \mathrm{rad} / \mathrm{s}$ while the wind speed is equal to $5.9 \mathrm{~m} / \mathrm{s}$. Green dots represent vortex particles and purple lines show the blades of the 2-m VAWT. Vortex particles show a repeating pattern. Their presence influences the flow angle of attack at each section of the blade.

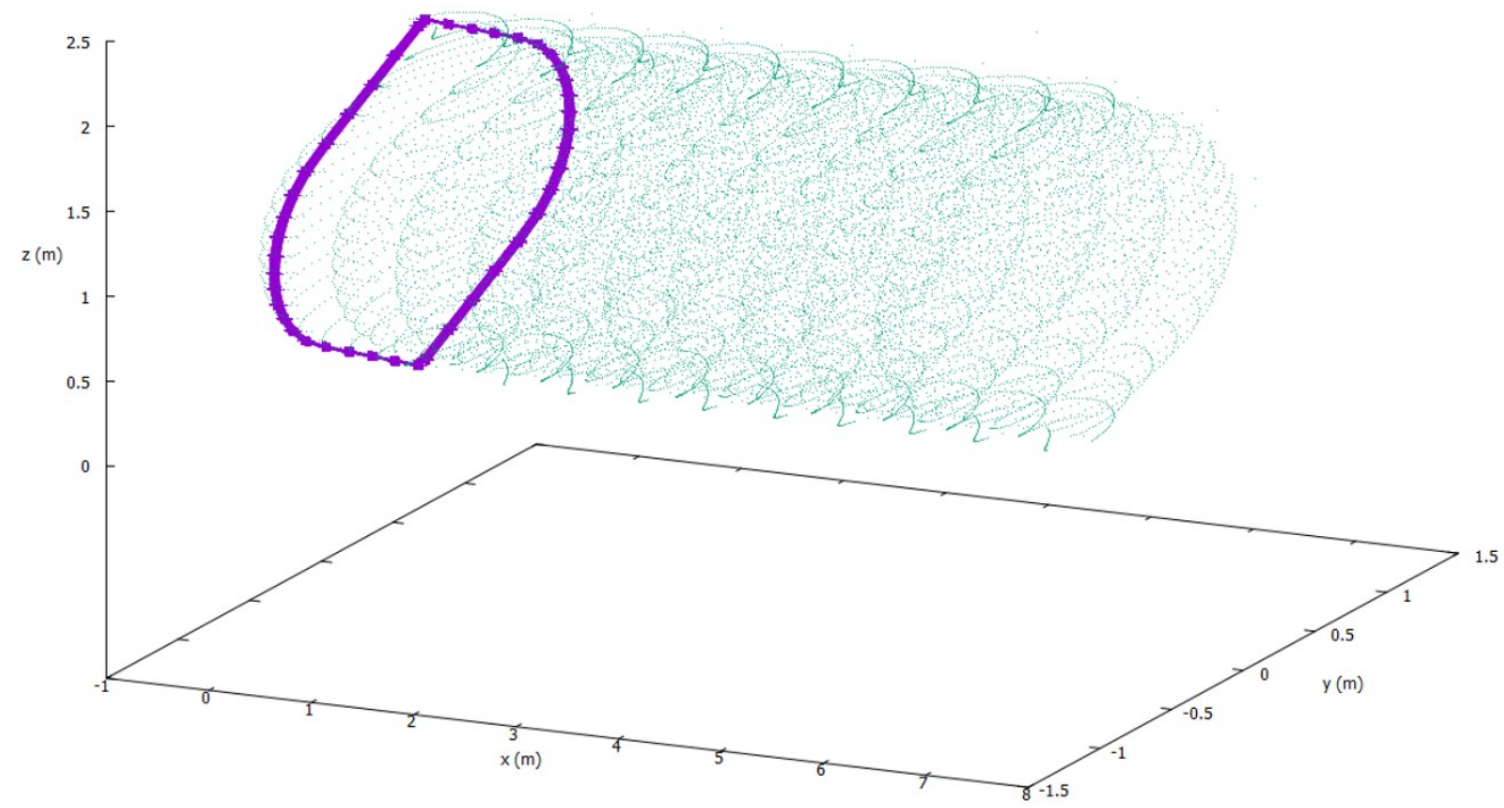

Figure 14 Wake Generated by Rotation of a 2-m Sandia Conventional Design Turbine. 


\section{Chapter 3:}

\subsection{Aeroacoustics Analysis}

The methodology used to analyze the aeroacoustic behavior of VAWTs is discussed in this chapter.

\subsection{Aeroacoustics Concepts}

Acoustic noise consists of longitudinal pressure waves travelling in air [14]. There are three main acoustic sources: dipoles, monopoles, and quadrupoles. A monopole point source can be represented by a pulsating sphere that emanates sound evenly in all directions and may be expressed by a fluctuating scalar quantity. A dipole source is made of two monopoles having same strengths that fluctuate out of phase, while there is a small gap between them. Quadrupole represents combination of four monopoles.

The sound produced by aerodynamic turbulence and moving surfaces are calculated using the FW-H equation, which is made from a surface distribution of monopoles and dipoles, and a volume distribution of quadrupole sources. Ffowcs Williams and Hawkings demonstrated that the thickness noise that is caused by a body's volume displacement (or thickness), may be expressed as a monopole acoustic source distribution. Loading noise, that is created by the distribution of aerodynamic force over a body's surfaces, may be described using dispersion of dipole acoustic sources. In a subsonic flow, compressibility effects cause quadrupole noise which has a lower noise compared to the thickness and loading noise [61] [89]. 


\subsection{Acoustic Formulation}

The quadrilateral surface panels of the rotor blades are regarded as point sources situated at the center of each panel in the geometric representation of the problem. All other geometric elements, such as the rotor tower, guy wires, ground, and any nearby structures, are neglected. Using this approach, aeroacoustics results are only reliant on the flow condition around the blades. In the experimental analysis of the aeroacoustics, geometric components like nearby structures and ground can affect the aeroacoustics. Rotor tower and guy wires make aeolian tones which are due to the unsteady wake that is shed from these components. The atmosphere is modelled as a homogenous medium with a constant pressure, density and free-stream velocity. Many environmental effects, such as acoustic attenuation, and input turbulence, are thus ignored.

There are two important time domains to consider in an acoustic modelling: source time $\tau$ and observer time t. An observer stationed at a location $\mathrm{x}$ and time $\mathrm{t}$ receives an acoustic emission released from the source at time $\tau$ and location y. Because of Doppler effects and variations in the acoustic radiation distance at different times, a discretized source time domain does not correlate to discretization of the observer's temporal domain that is evenly spaced [90]. As a result, the observer time has often a higher resolution compared to source (aerodynamic) time, allowing for the capturing of higher frequency of acoustic signals.

An acoustic analogy formulation is utilized to predict the perturbation pressure in the observer location for acoustic sources. Following equation is used to calculate the time that acoustic pressure is experienced by the observer [89]: 


$$
t_{a d v}=\tau_{e}+\frac{R}{C_{0}}
$$

where $\tau_{e}, \mathrm{R}$ and $C_{0}$ are defined as the emission source time, acoustic radiation distance at emission time $\tau_{e}$, and speed of sound in the undisturbed medium, respectively.

\section{Formulation 1C}

Rotors in the wind turbines can be considered as moving acoustic sources in a moving medium which is the wind that is uniform in the entire domain [63]. Formulation $1 \mathrm{C}$ was proposed by Najafi-Yazdi et al. [63], which was an extension to the original $\mathrm{FW}-\mathrm{H}$ equation (Formulation 1A). Formulation $1 \mathrm{C}$ is obtained by utilizing the convective wave equation which considers the impact of moving medium that is ignored by other formulations of the FW-H equation. A couple of terms is suggested by Najafi-Yazdi in the Formulation $1 \mathrm{C}$, which are introduced in this sub-section. There are further information and details about the Formulation 1C in the Appendix B. Formulation 1C is obtained for the situation in which medium moves evenly in just one direction, and all formulas provided within this study are based on that assumption.

The scalar expression $Q_{n}$ is used for thickness sources, whereas the vector expression $L_{n i}$ is used for loading sources. At the source locations, these terms are computed as follows:

$$
\begin{gathered}
Q_{n}=\rho_{0}\left(v_{i}-U_{\infty i}\right) \hat{n}_{i}, \\
L_{n i}=p \hat{n}_{i} .
\end{gathered}
$$

In the above equation, $v$ is the surface velocity in a direction normal to the surface and $p$ is the surface's perturbation pressure. Between a source at point $\mathrm{y}$ and an observer at $\mathrm{x}$, acoustic radiation distance " $R$ " can be computed using the following equation [63]: 


$$
R=\frac{-M_{\infty}\left(x_{1}-y_{1}\right)+R^{*}}{\hat{\beta}^{2}}
$$

where $R^{*}$ and $\hat{\beta}$ that is the Prandtl-Glauert factor can be computed using the following equations: [63]

$$
\begin{gathered}
R^{*}=\sqrt{\left(x_{1}-y_{1}\right)^{2}+\dot{\beta}^{2}\left[\left(x_{2}-y_{2}\right)^{2}+\left(x_{3}-y_{3}\right)^{2}\right]}, \\
\dot{\beta}=\sqrt{1-M_{\infty}{ }^{2}} .
\end{gathered}
$$

Based on [63], the thickness and loading perturbation pressures are determined using the following generic expressions:

$$
\begin{gathered}
4 \pi \dot{p}_{T}\left(x, t_{a d v}\right)=\left[\frac{\partial}{\partial t}+U_{\infty} \frac{\partial}{\partial x_{1}}\right] \int_{-\infty}^{t} \int_{\mathbb{R}^{3}} Q_{j} n_{j} \delta(f) \frac{\delta(g)}{R^{*}} d^{3} y d \tau \\
4 \pi \dot{p}_{L}\left(x, t_{a d v}\right)=-\frac{\partial}{\partial x_{i}} \int_{-\infty}^{t} \int_{\mathbb{R}^{3}} L_{i j} n_{j} \delta(f) \frac{\delta(g)}{R^{*}} d^{3} y d \tau
\end{gathered}
$$

where $\delta$ is the Kronecker delta function and the parameter $\mathrm{g}$ is described as [63]

$$
\mathrm{g}=\tau-t+\frac{R}{c_{0}}
$$

Far-field perturbation pressures can be obtained using the following equations: 


$$
\begin{aligned}
4 \pi \dot{p}_{T}\left(x, t_{a d v}\right) & =\sum_{k=1}^{N}\left[\left(\frac{\dot{Q}_{n}}{R^{*}\left(1-M_{R}\right)^{2}}-\frac{\partial R^{*}}{\partial \tau} \frac{Q_{n}}{R^{* 2}\left(1-M_{R}\right)^{2}}\right.\right. \\
& \left.+\frac{Q_{n}}{R^{*}\left(1-M_{R}\right)^{3}} \frac{\partial M_{R}}{\partial \tau}\right)-M_{\infty}\left(\frac{\dot{\tilde{R}}_{1} Q_{n}+\tilde{R}_{1} \dot{Q}_{n}}{R^{*}\left(1-M_{R}\right)^{2}}\right. \\
& -\frac{\partial R^{*}}{\partial \tau} \frac{\tilde{R}_{1} Q_{n}}{R^{* 2}\left(1-M_{R}\right)^{2}}+\frac{\partial M_{R}}{\partial \tau} \frac{\tilde{R}_{1} Q_{n}}{R^{*}\left(1-M_{R}\right)^{3}} \\
& -U_{\infty}\left(\frac{\tilde{R}^{*}{ }_{1} Q_{n}}{R^{* 2}\left(1-M_{R}\right)}\right]_{k} S_{k}, \\
4 \pi \dot{p}_{L}\left(x, t_{a d v}\right) & =\sum_{k=1}^{N}\left[\frac { 1 } { c _ { 0 } } \left(\frac{\dot{L}_{n i} \tilde{R}_{i}+L_{n i} \tilde{R}_{1}}{R^{*}\left(1-M_{R}\right)^{2}}-\frac{\partial R^{*}}{\partial \tau} \frac{L_{n i} \tilde{R}_{i}}{R^{* 2}\left(1-M_{R}\right)^{2}}\right.\right. \\
& \left.\left.+\frac{\partial M_{R}}{\partial \tau} \frac{L_{n i} \tilde{R}_{i}}{R^{*}\left(1-M_{R}\right)^{3}}\right)+\frac{L_{n i} \tilde{R}_{i}^{*}}{R^{* 2}\left(1-M_{R}\right)}\right]_{k} S_{k}
\end{aligned}
$$

where $M_{R}$ is defined as the surface's Mach number in the direction of radiation.

$$
M_{R}=\frac{1}{C_{0}} v_{i} \tilde{R}_{i}
$$

Also, according to [63], $\tilde{R}_{i}$ and $\tilde{R}_{i}{ }^{*}$ which are scalar gradients can be defined as following:

$$
\begin{aligned}
& \tilde{R}_{i}^{*}=\frac{\partial}{\partial x_{i}} R^{*} \Rightarrow \tilde{R}_{1}^{*}=\frac{x_{1}-y_{1}}{R^{*}}, \tilde{R}_{2}{ }^{*}=\dot{\beta}^{2} \frac{x_{2}-y_{2}}{R^{*}}, \tilde{R}_{3}{ }^{*}=\hat{\beta}^{2} \frac{x_{3}-y_{3}}{R^{*}} \\
& \tilde{R}_{i}=\frac{\partial}{\partial x_{i}} R \Rightarrow \tilde{R}_{1}=\frac{1}{\hat{\beta}^{2}}\left(-M_{\infty}+\tilde{R}_{1}^{*}\right), \tilde{R}_{2}=\frac{x_{2}-y_{2}}{R^{*}}, \tilde{R}_{3}=\frac{x_{3}-y_{3}}{R^{*}} .
\end{aligned}
$$

The thickness and loading formulas for the non-penetrable version of Formulation 1C may be equal to the thickness and loading formulas for the non-penetrable form of Formulation 1A stated by Farassat in [91] for the particular situation of zero mean flow velocity. By existence of mean flow and a low Mach number $\left(M_{\infty}<0.1\right)$, results derived from Formulations $1 \mathrm{~A}$ and $1 \mathrm{C}$ are approximately equal. 
In the Equations (3.10) and (3.11), the terms that includes $R^{*^{2}}$ in the denominator ("near-field") decrease faster when acoustic radiation distance increases compared to the terms include $R^{*}$ in the denominator ("far-field").

Perturbation pressures achieved by using Equations (3.10) and (3.11), are not the same as the corresponding loading and acoustic pressures. A time averaged factor $\bar{p}$ and a fluctuating factor $\tilde{p}$ can be considered for each perturbation pressure $\dot{p}$. The fluctuating factor can be considered as an acoustic pressure:

$$
\tilde{p}(x, t)=\dot{p}(x, t)-\bar{p}(x)
$$

In the approach used in this thesis, total perturbation and acoustic pressures are equal to the summation of the respective thickness and loading pressures.

According to [63], the amount of acoustic pressure is frequently stated as a sound pressure level (SPL) in decibels and as an RMS average of acoustic pressure. These values are determined as follows for each given observer [92]:

$$
\begin{gathered}
\tilde{p}_{r m s}=\sqrt{\overline{(\tilde{p})^{2}}} \\
\mathrm{SPL}=20 \log _{10}\left(\frac{\tilde{p}_{r m s}}{p_{r e f}}\right)
\end{gathered}
$$

where $p_{\text {ref }}$ is equal to $2 \times 10^{-5} \mathrm{~Pa}$ and SPL is defined in terms of decibels $(\mathrm{dB})$. Regularly, notation " $\mathrm{dB}$ re $2 \times 10^{-5} \mathrm{~Pa}$ " is used to show that the decibels are established relatively to the pressure $2 \times 10^{-5} \mathrm{~Pa}$.

The aeroacoustics code is written separately from the aerodynamic code in FORTRAN 90. Both codes were developed at the University of Athens. The aeroacoustics code runs independently from the aerodynamic code, this approach can help in managing computational time and expense as aeroacoustics analysis can be executed for different 
observer positions and time resolution without having to re-execute the aerodynamic simulation which needs considerably more time and computational expense. The user specifies the position and time resolution for all observers and the aeroacoustics source information, which was obtained using the aerodynamic code. Moreover, the aeroacoustic code has been verified and validated against separate experimental results by the University of Athens in the aeroacoustic analysis of the helicopters [93], hence current aeroacoustic results should be dependable. 


\section{Chapter 4:}

\subsection{Results and Discussion}

In this chapter, aerodynamic and aeroacoustic results for different VAWT sizes and configurations are provided using the methods described in the previous chapters. In the first section, convergence analysis is performed using azimuth angle, turbine's revolution, number of panels in each blade and number of nodes in each panel, as variables. In the second part, aerodynamic results for the 2-m and 17-m Sandia VAWT are provided and a comparison between different configurations is given. In the final section, aeroacoustics predictions for different turbine configurations and sizes are performed and results are discussed. It should be mentioned that studies are performed at atmospheric conditions with air density of $\rho_{0}=1.205 \mathrm{~kg} / \mathrm{m}^{3}$ and speed of sound $c_{0}=340 \mathrm{~m} / \mathrm{s}$. The following parameters are used and repeated here for the sake of convenience:

Parameter

Power Coefficient

Tip Speed Ratio

Solidity

\section{Equations}

$$
C_{p}=\frac{P}{\frac{1}{2} A_{s} \rho U_{\infty}^{3}}
$$

$$
T S R=\lambda_{T}=\frac{r_{T} \omega_{T}}{U_{\infty}}
$$

$$
\sigma_{T}=\frac{N_{b} c_{T} L_{b}}{A_{S}}
$$

where $P$ is the average power generated by the turbine, $\rho$ is the fluid density, $A_{s}$ is the swept area, $U_{\infty}$ is the wind velocity, $r_{T}$ is the rotor radius between the axis of rotation and the outermost point of the rotor blade, $\omega_{T}$ is the rotor angular velocity, $N_{b}$ is the number 
of rotor blades, $c_{T}$ is the chord length, which is constant along the span for each blade (for VAWTs), and $L_{b}$ is the whole length of the blade.

\subsection{Convergence Analysis}

Before obtaining numerical results for different configurations of VAWTs, it was important to ensure that simulations are fully converged and accurate. In this part, a study is done on the impact of the azimuth angle increment on the power coefficient in the 2-m and 17-m VAWTs.

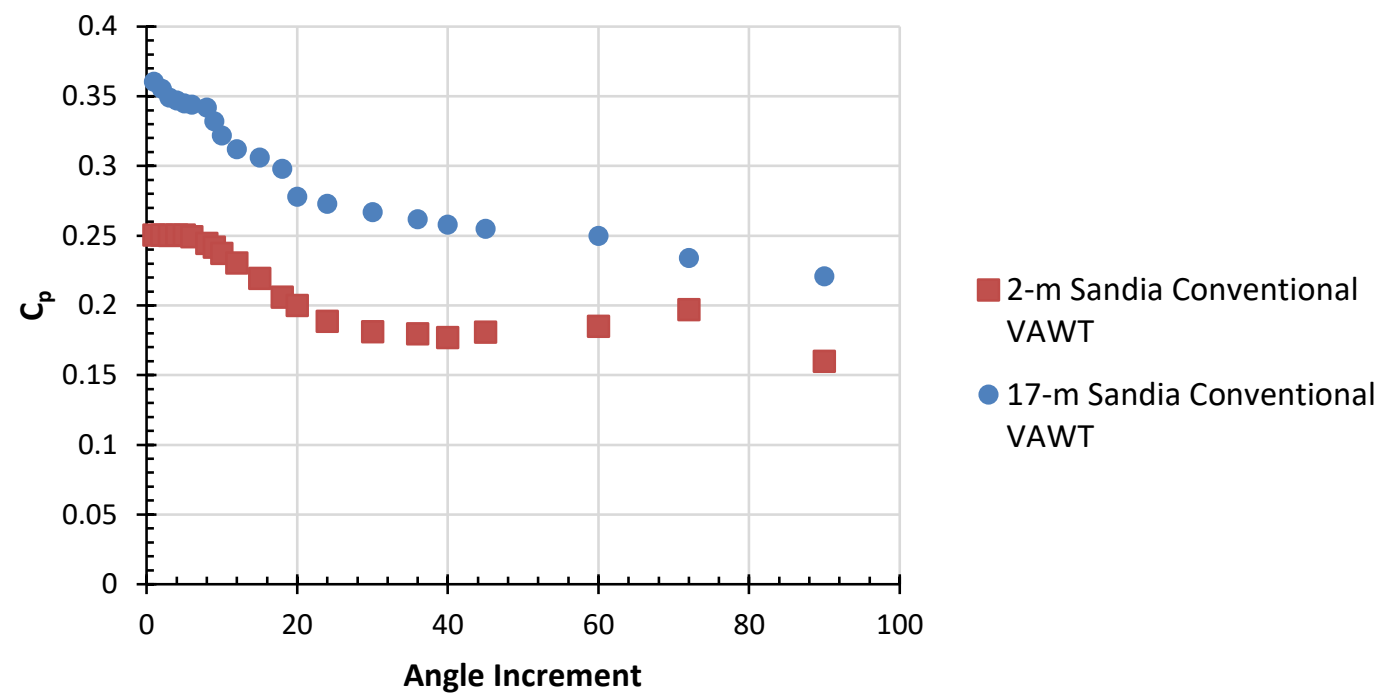

Figure 15 Impact of the Azimuth Angle Increment on the Power Coefficient.

Figure 15 shows the effect of the azimuth angle increment (equivalent to a time step) on the power coefficient. For example, if an increment angle of 2 degrees is selected, a completed turbine revolution having 360 degrees will require 180 timesteps to be completed. As it is evident in the figure, the power coefficient variation is relatively small for increment angles less than 10 degrees, but considerably higher for increment angles 
bigger than 15 degrees. In the $17-\mathrm{m}$ VAWT, there is a small variation in the power coefficient for a small value of angle increment, although the variation is smaller than $2 \%$, mainly due to higher fluctuations in aerodynamic factors when compared against the 2-m VAWT. In this thesis, an increment angle of 4 degrees is adopted, combining relatively accurate aerodynamic results while maintaining reasonable computational time and cost.

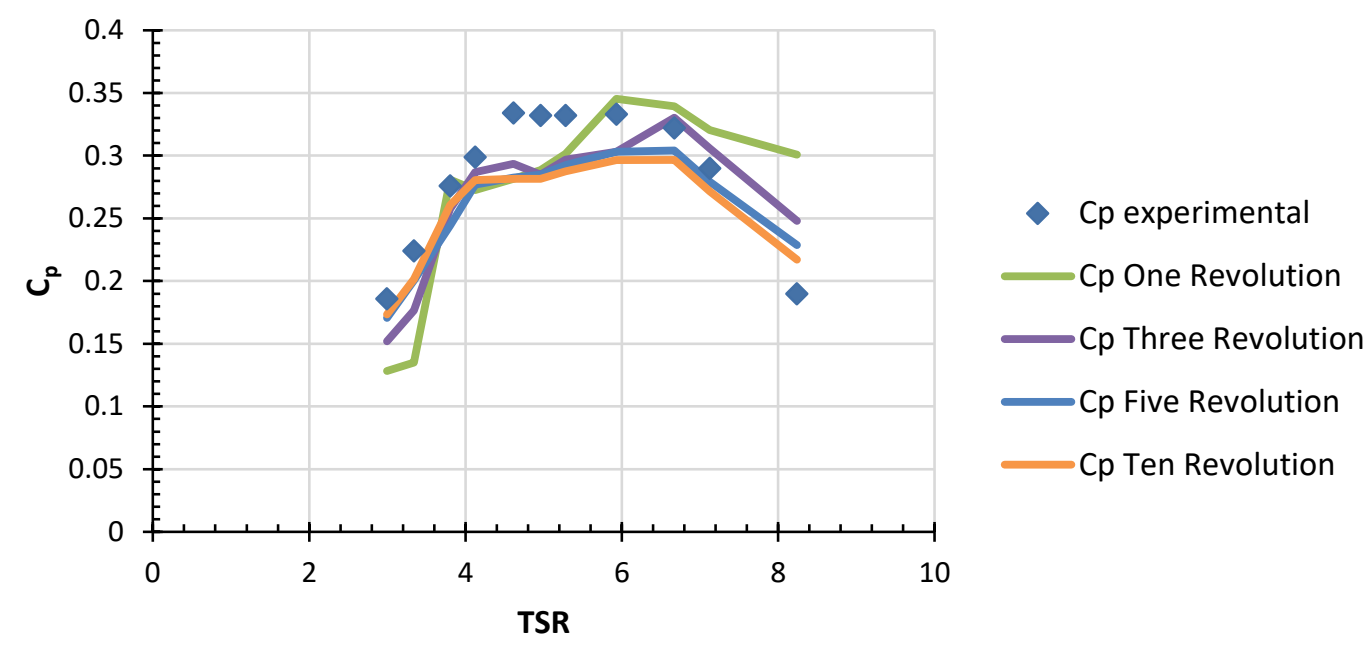

Figure 16 Effect of the Turbine's Number of Revolutions on the Power Coefficient.

The power coefficient of the 17-m Sandia conventional VAWT is shown in the figure above as a function of TSR for different number of revolutions. It is reasonable to assume that increasing the number of revolutions would improve data accuracy, and the figure above can substantiate the statement. The higher the number of rotations, the closer the aerodynamic results are to available experimental data. One important point is that higher number of revolutions require more computational time and cost, and a balance between accuracy of the output data and the computational expenses is needed. Following the figure, five revolutions is adopted in the present work, since relatively good accuracy in the aerodynamic results is obtained while preventing higher computational time and cost. 
Both influences of the number of panels in each blade and the number of nodes in each panel are discussed in the following figure for the 2-m conventional VAWT:
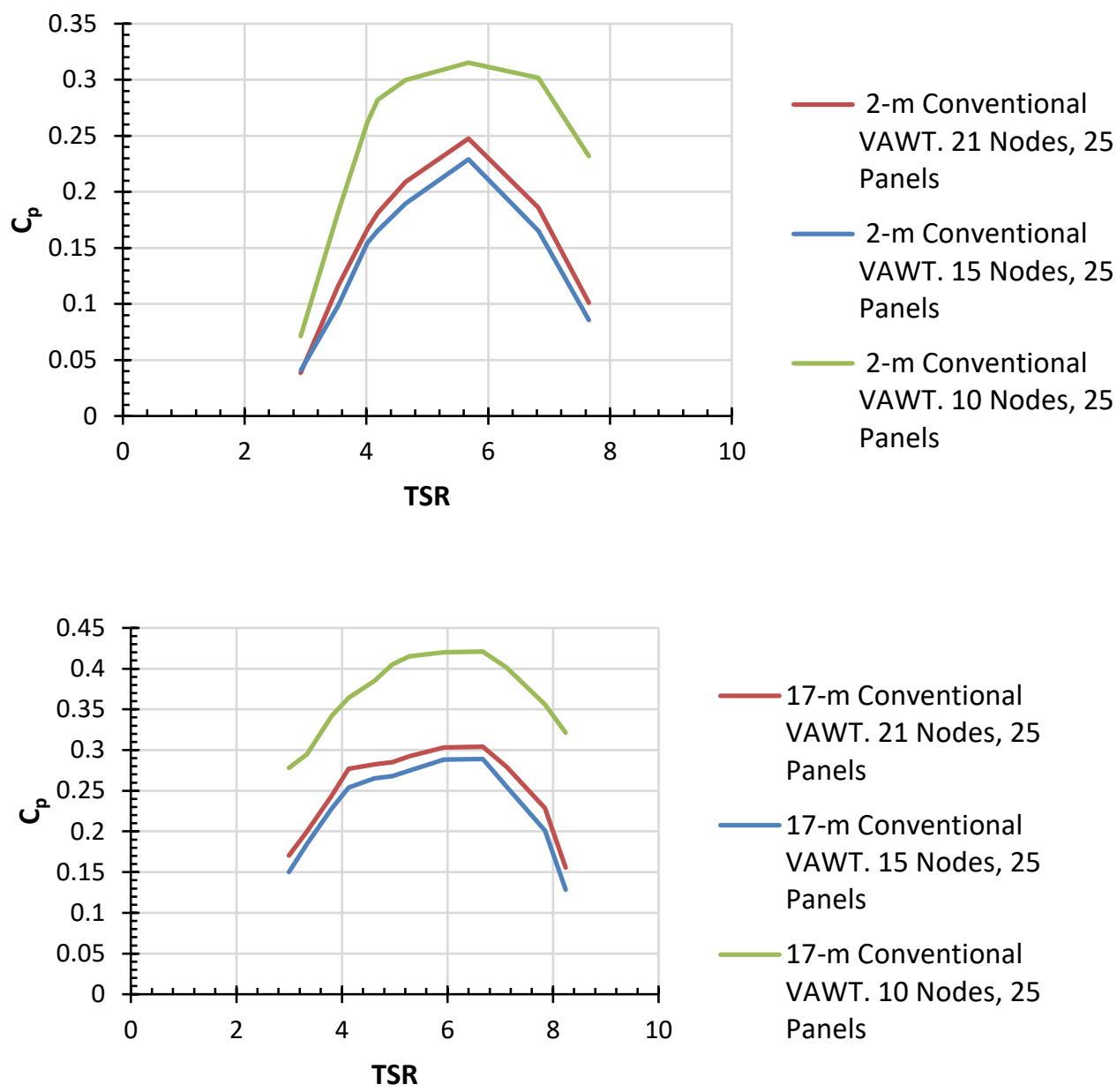

Figure 17 Effect of the Number of Nodes in Each Blade, in Power Coefficient.
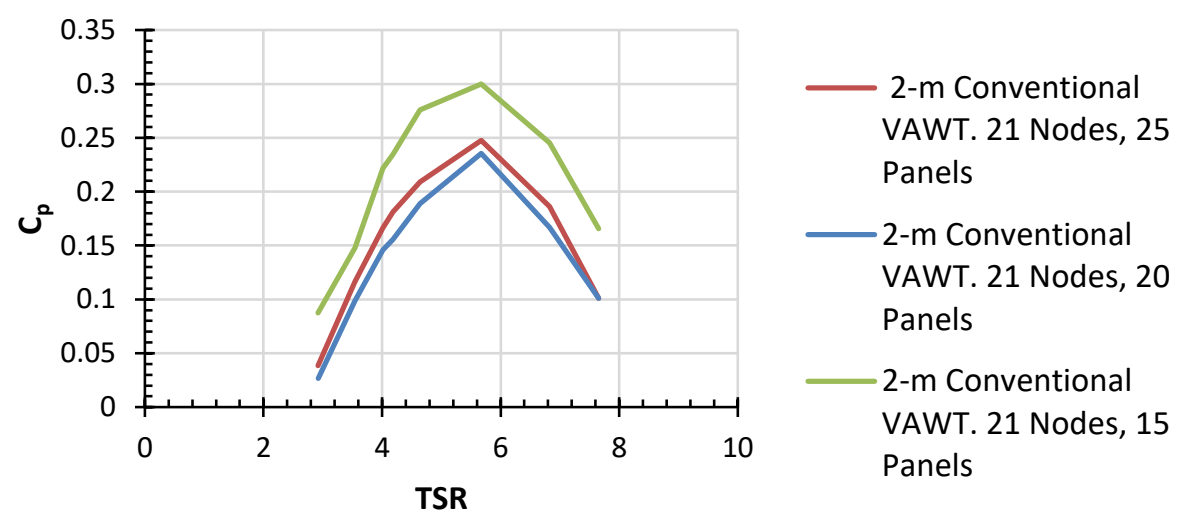


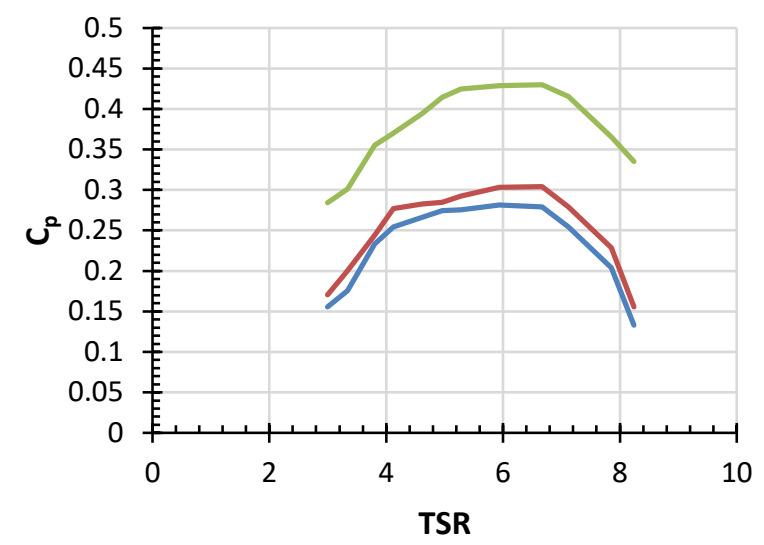

17-m Conventional

VAWT. 21 Nodes, 25

Panels

17-m Conventional

VAWT. 21 Nodes, 20

Panels

17-m Conventional VAWT. 21 Nodes, 15

Panels

\section{Figure 18 Effect of the Number of Panels on Each Blade Segment, in Power}

\section{Coefficient.}

In Figure 17, the number of panels in each blade segment is kept constant (25) while the number of nodes in each blade is decreased $(21,15$, and 10). Although there is less discrepancy between 21 and 15 nodes, the usage of 10 nodes clearly affects the simulated efficiency of the turbine. In Figure 18, the number of nodes in a blade is kept constant while the number of panels in each blade segment varied $(25,20$, and 15). Simulated performance for 25 and 20 panels is similar but results for 10-panels are significantly different. This analysis indicates that 21 nodes and 25 panels seem adequate for the current simulations.

\subsection{Aerodynamic Prediction of Different VAWT Models}

Although VAWT shifted configuration was proposed by Hilewit et al. [9], solidity considerations were not taken-into-account at the time. For fairer comparison between turbines, solidity is maintained constant in the current thesis by changing the chord length of the airfoil when the blade length is changed. 
VAWT shifting can be performed by keeping the turbine height and swept area constant and shortening the length of the blades as shown in Figure 19 (referred to as STSVAWT or Shifted-Troposkien-Shape VAWT).

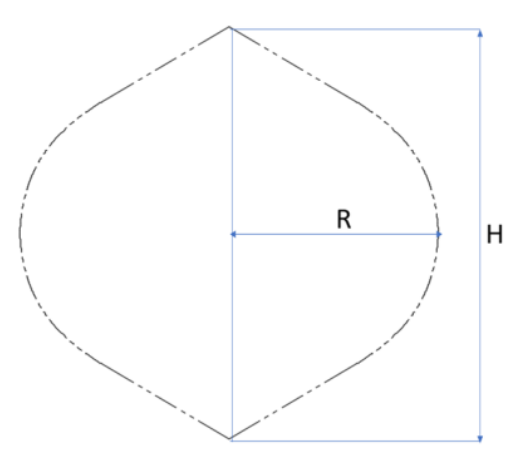

(a)

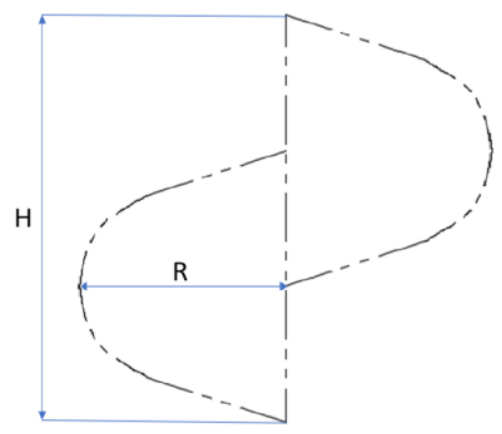

(b)

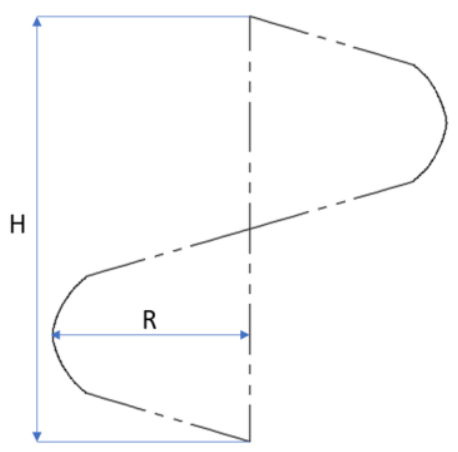

(c)

Figure 19 Geometry Schematics: a) Conventional Troposkien-Darrieus VAWT, b) $50 \%$ STS-VAWT (Shifted-Troposkien-Shape VAWT), and c) 100\% STS-VAWT.

\subsubsection{2-m Configurations}

In Table 2, parameters and geometrical characteristics for the first set of study, which includes the 2-m conventional Sandia VAWT, 2-m 50\% and 100\% STS models (see Figure 19), are provided. Turbine radius, height, swept area, and solidity ratio are kept constant while blade length is shortened and airfoil chord length is increased. Numerical aerodynamic analysis of these configurations was performed using GENUVP. 
Table 2 Geometric Characteristics of 2-m Sandia STS-VAWT Configurations.

\begin{tabular}{|c|c|c|c|}
\hline Parameters & 2-m Conventional & 2-m 50\% STS-VAWT & 2-m 100\% STS-VAWT \\
\hline Number of Blades & 2 & 2 & 2 \\
\hline Turbine Radius (m) & 0.979 & 0.979 & 0.979 \\
\hline Turbine Height (m) & 2 & 2 & 1 \\
\hline Blade Height (m) & 2 & 1.33 & 2 \\
\hline Blade Length (m) & 2.97 & 2.59 & 0.130 \\
\hline Swept Area (m ${ }^{2}$ ) & 2.59 & 2.59 & 0.20 \\
\hline Chord Length (m) & 0.08815 & 0.10108 & 0012 \\
\hline Solidity Ratio & 0.20 & 0.20 & \\
\hline Airfoil (NACA) & 0012 & 0012 & \\
\hline
\end{tabular}

Figure 20 and Figure 21 show the 2-m VAWT simulation results of power coefficient as function of TSRs (Tip Speed Ratios) for two rotational velocities, 27.9 and $41.9 \mathrm{rad} / \mathrm{s}$, respectively. Notice that an increase in rotational speed from 27.9 to $41.9 \mathrm{rad} / \mathrm{s}$ created a decrease in the overall power coefficients for all turbines in the current simulations. $50 \%$ STS-VAWT has the highest peak power for both rotational velocities and better performance at higher TSRs (lower wind speeds), while slightly underperforming for low TSRs (approximately TSR < 4.5) when compared against the conventional TroposkienDarrieus VAWT by Sandia National Laboratories. This performance improvement is expected due to a decrease in BWI (Blade Wake Interaction) at high TSRs. The wake from the equator plane (power generating region) of the first blade interacts less with the equator plane of the second blade due to the blade-shifting. 100\% STS-VAWT shows the weakest performance of the three simulated turbines. It is conjectured here that the weak performance reflects the reduction of blade length, thus producing less power 
comparatively to the same swept area, despite further reduction in BWIs when compared against the other two configurations.

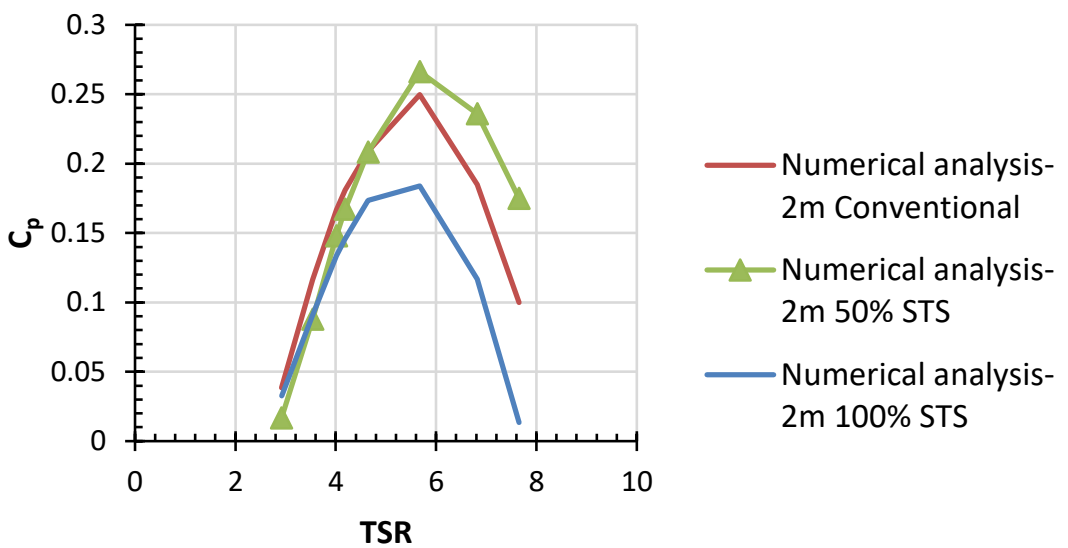

Figure 20 Turbine Performance for 2-m Conventional, 2-m 50\% STS-VAWT (Shifted Troposkien Shape - VAWT), and 2-m 100\% STS-VAWT at $27.9 \mathrm{rad} / \mathrm{s}$.

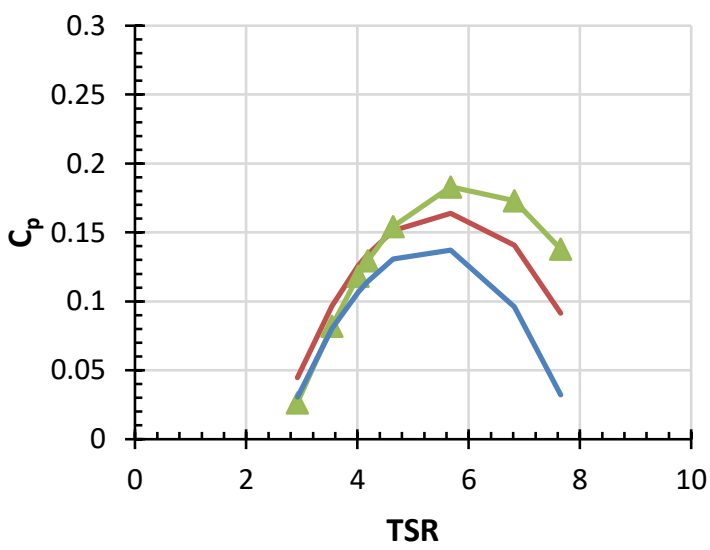

- Numerical Analysis- 2m Conventional

- Numerical Analysis- 2m $50 \%$ STS

Numerical Analysis- 2m $100 \%$ STS

Figure 21 Turbine Performance for 2-m Conventional, 2-m 50\% STS-VAWT, and 2-m 100\% STS-VAWT Configurations at $41.9 \mathrm{rad} / \mathrm{s}$. 


\subsubsection{7-m Configurations}

The numerical studies provided in the Section 4.3.1 are repeated for 17-m VAWTs to observe the aerodynamic behavior for a larger turbine. 17-m Sandia Conventional VAWT is compared to the $50 \%$ and $100 \%$ STS configurations. The turbine characteristics are provided in the following table. Turbine height, swept area, and solidity ratios are kept constant, while blade length and chord length are varied between different configurations.

Table 3 Geometric Characteristics of 17-m STS-VAWT.

\begin{tabular}{|c|c|c|c|}
\hline Parameters & $\begin{array}{c}\text { 17-m Conventional } \\
\text { VAWT }\end{array}$ & 17-m 50\% STS-VAWT & 17-m 100\% STS-VAWT \\
\hline Number of Blades & 2 & 2 & 2 \\
\hline Turbine Radius (m) & 8.366 & 8.366 & 8.366 \\
\hline Turbine Height (m) & 17 & 17 & 17 \\
\hline Blade Height (m) & 17 & 20.8294 & 19.20 \\
\hline Blade Length (m) & 24.71 & 187.10 & 187.10 \\
\hline Swept Area ( $m^{2}$ ) & 187.10 & 0.629 & 0.682 \\
\hline Chord Length (m) & 0.53 & 0.14 & 0.14 \\
\hline Solidity Ratio & 0.14 & 0015 & 0015 \\
\hline Airfoil (NACA) & 0015 & & \\
\hline
\end{tabular}

As shown in Figure 23 and Figure 22, 50\% STS-VAWTs have a higher peak in power coefficient and better performance than conventional VAWTs for high TSRs, while underperforming for lower TSRs (higher wind speeds), particularly for $4<$ TSR $<6$, for both rotational speeds (4.4 and $5.5 \mathrm{rad} / \mathrm{s})$. Notice that this increase in rotational speed does not affect the overall efficiency of the 17-m turbines considerably. Notice also that the rotational speeds ( 4.4 and $5.5 \mathrm{rad} / \mathrm{s}$ ), common for bigger VAWTs, are smaller than the ones 
for the 2-m turbines (27.9 and $41.9 \mathrm{rad} / \mathrm{s}) .17-\mathrm{m}$ turbines have higher power coefficients throughout the range of TSRs simulated in the present work, when compared to the 2-m turbines. 100\% STS-VAWT underperforms considerably when compared against the conventional and the 50\%-VAWT for both rotational speeds, likely due to reduction in power-producing blade length, as discussed before.

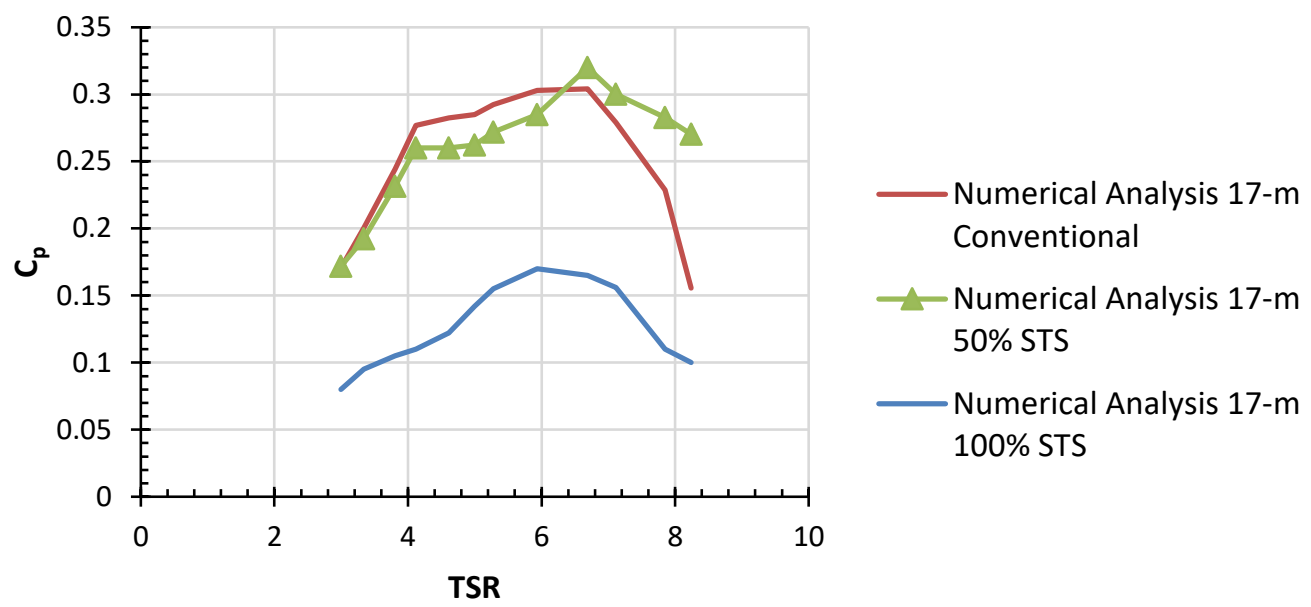

Figure 22 Turbine Performance for 17-m Conventional, 17-m 50\% STS-VAWT (Shifted Troposkien Shape VAWT), and 17-m 100\% STS-VAWT at $4.4 \mathrm{rad} / \mathrm{s}$.

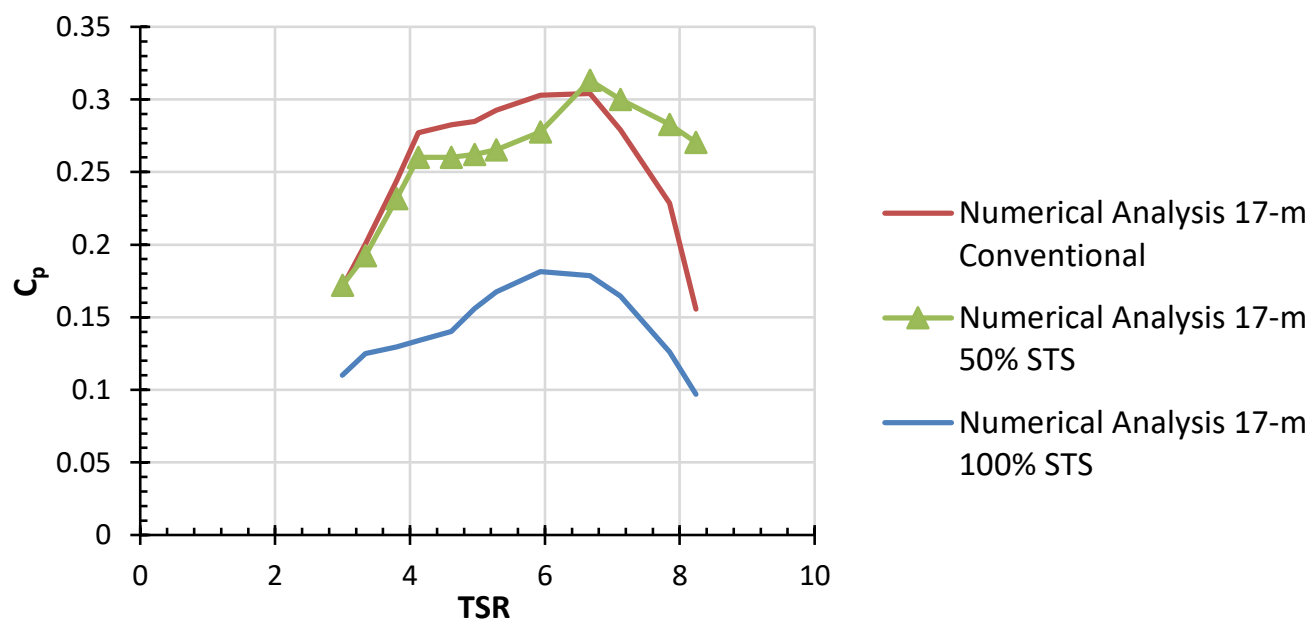

Figure 23 Turbine Performance for 17-m Conventional, 17-m 50\% STS-VAWT (Shifted Troposkien Shape VAWT), and 17-m 100\% STS-VAWT at $5.5 \mathrm{rad} / \mathrm{s}$. 
From both 2-m and 17-m VAWT aerodynamic analyses, it is apparent that the 50\% STSVAWT becomes an alternative to the conventional VAWT, when overall power efficiency and some cost savings are concerned.

\subsection{Aeroacoustics}

In this part, aeroacoustics results are provided using the Formulation 1C of Ffowcs Williams - Hawkings equation, which has been discussed in Chapter 3. The current simulations do not consider the existence of a ground level as a simplification to avoid reflections (the turbine is floating and the analysis is performed). Acoustic analysis is performed for the conventional and 50\% STS-VAWT configurations and results are compared to each other to have a better understanding of acoustic behavior of each turbine. For the 2-m configurations, a vertical plane $(12.0 \mathrm{~m} \times 12.0 \mathrm{~m})$ placed $2.5 \mathrm{~m}$ downstream from turbine's axis of rotation and used initially for the acoustic analysis as shown in

Figure 24. Notice that the bottom of the turbine is located at the origin $(0,0,0)$.

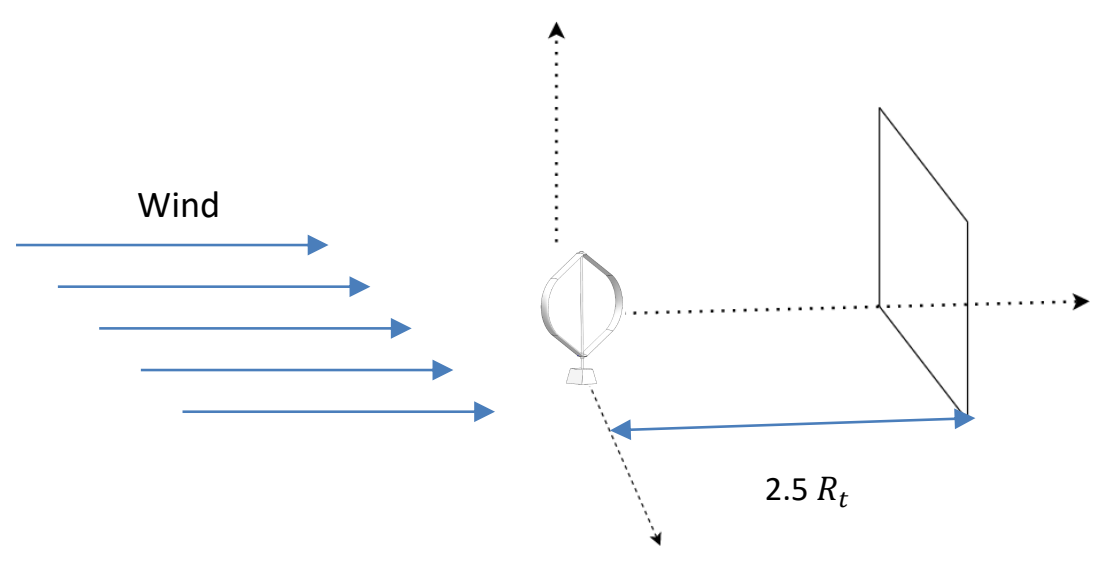

Figure 24 Location of the Turbine and Plane where Acoustic Analysis is Performed. 
Table 4 Geometric Characteristics for the 2-m VAWTs.

\begin{tabular}{|c|c|c|}
\hline Configuration & 2-m Conventional VAWT & 2-m 50\% STS-VAWT \\
\hline Blade Shape & Troposkien & Troposkien \\
\hline Airfoil & NACA 0012 & NACA0012 \\
\hline Rotor Diameter (m) & 0.979 & 0.979 \\
\hline Rotor Height (m) & 2 & 2 \\
\hline Chord Length (m) & 0.08815 & 27.946 \\
\hline Rotational Speed (Rad/s) & 27.946 & 4.824 \\
\hline Wind Speed (m/s) & 4.824 & 5.675 \\
\hline Tip Speed Ratio & 5.675 & \\
\hline
\end{tabular}

Using aerodynamic results at TSR $=5.675$ for $27.9 \mathrm{rad} / \mathrm{s}$, provided previously, aeroacoustic analysis is performed for the first set of the turbines which includes the 2-m conventional design and its $50 \%$ shifted configuration with geometrical parameters presented in the previous table (Table 4). Acoustic results (Sound Pressure Level in decibels) on the plane that is defined above are provided in the following figure: 


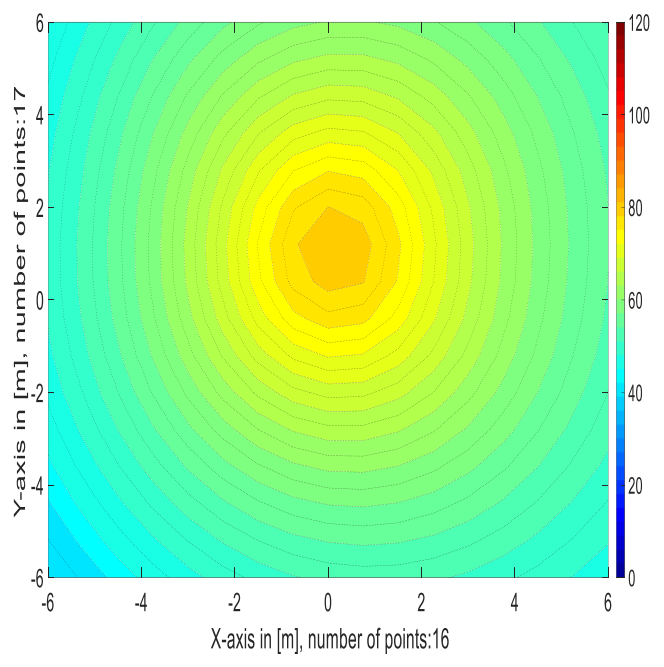

(a)

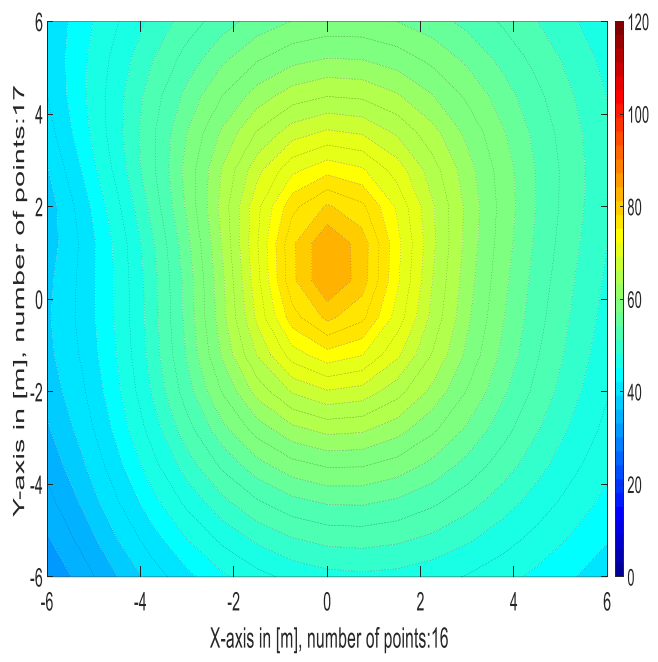

(b)

Figure 25 Acoustic Map at a Plane 2.5 m Downstream from the Axis of the Turbine: a) 2-m Conventional VAWT and b) 2-m 50\% STS-VAWT.

According to the figure above, 2-m conventional VAWT has its maximum sound intensity above the center of the plane where the turbine is located with a value of $80 \mathrm{~dB}$, while the sound intensity decreases away from the center, as expected. 50\% STS-VAWT generates 
more peak noise $(85 \mathrm{~dB})$ when compared to the conventional VAWT, but the sound intensity decreases faster with distance from the peak, likely because of the smaller blade size.

Acoustic pressure is calculated at different frequencies at a point near the center of the plane as shown in Figure 26, for the 2-m VAWT configurations. Figure 27 shows the SPL at different frequencies at the specified point. 50\% STS-VAWT has similar signature when compared against conventional VAWT. $50 \%$ STS design has a higher peak (90 dB) when compared to the conventional design $(85 \mathrm{~dB})$, and both figures have a peak around a frequency that is equal to the rotational frequency of turbine $(27.9 \mathrm{rad} / \mathrm{s}$ or $4.4 \mathrm{~Hz})$, followed by additional valley/peak as multiple of this frequency.

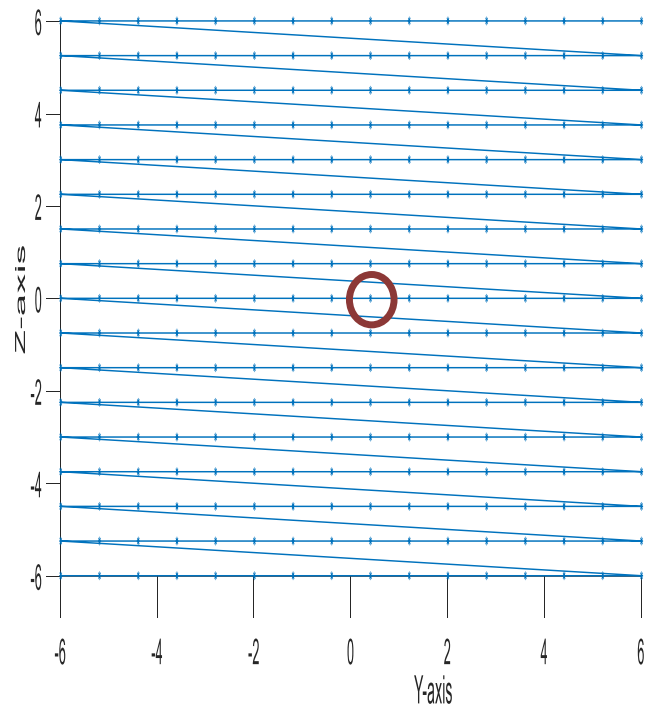

Figure 26 Exact Location of the Point that Acoustic Pressure for Different Frequencies is Calculated on, in the 2-m VAWT Configurations. 


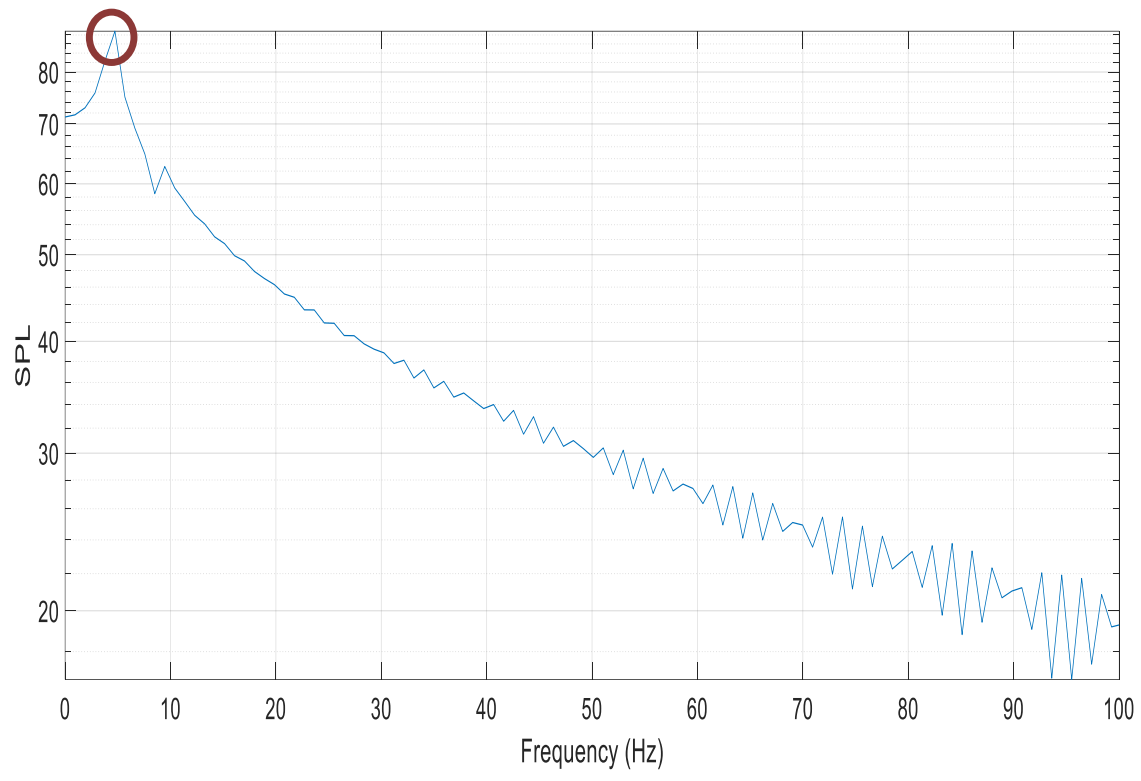

(a)

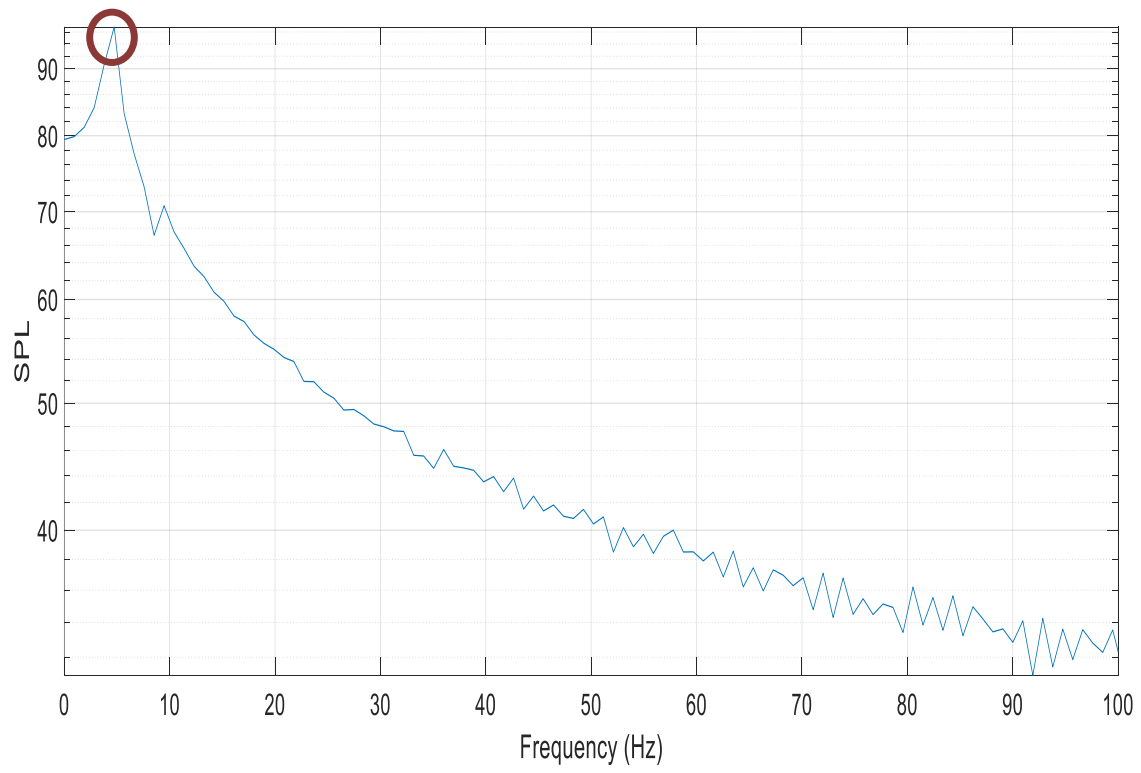

(b)

Figure 27 Acoustic Pressure vs. Frequency: a) 2-m Conventional VAWT and b) 2-m $50 \%$ STS-VAWT. 
Geometric characteristics for the 17-m VAWTs, which includes the 17-m Sandia Conventional VAWT and $17-\mathrm{m} 50 \%$ configuration at $(\mathrm{TSR}=7.12$ and $5.5 \mathrm{rad} / \mathrm{s})$, are provided in the following table:

Table 5 Geometric Characteristics for the 17-m VAWTs.

\begin{tabular}{|c|c|c|}
\hline Configuration & $\begin{array}{l}\text { 17-m Conventional } \\
\text { VAWT }\end{array}$ & 17-m 50\% STS-VAWT \\
\hline Blade Shape & Troposkien & Troposkien \\
\hline Airfoil & NACA 0015 & NACA0015 \\
\hline Rotor Diameter (m) & 16.732 & 16.732 \\
\hline Rotor Height (m) & 17 & 17 \\
\hline Chord Length (m) & 0.53 & 0.629 \\
\hline Rotational Speed (rad/s) & 5.49 & 5.49 \\
\hline Wind Speed $(\mathrm{m} / \mathrm{s})$ & 6.4443 & 6.4443 \\
\hline Tip Speed Ratio & 7.12 & 7.12 \\
\hline
\end{tabular}

Aeroacoustics analysis is performed for the turbines above, using the aerodynamic data obtained from GENUVP. Same as the 2-m VAWT, a vertical plane $(60.0 \mathrm{~m} \times 60.0 \mathrm{~m})$ is placed at $2.5 R_{t}$ downstream from turbine's axis of rotation. Notice that $R_{t}$ is account for the turbine's radius. $17-\mathrm{m} 50 \%$ STS-VAWT generates more noise with a peak of $105 \mathrm{~dB}$, (see Figure 28.b) when compared against the 17-m conventional VAWT (95 dB, Figure 28.a). More acoustic noise is generated by $17-\mathrm{m}$ turbines simulated here, when compared against the 2-m VAWTs for both conventional and 50\% STS-VAWT. Figure 29 shows the point near the center of the plane where the sound pressure level for different frequencies is calculated. According to Figure 30, major oscillation in total pressure occurs at $17(\mathrm{~Hz})$ for the $17-\mathrm{m}$ conventional VAWT, while for the $17-\mathrm{m}$ 50\% STS model, major 
fluctuations start at $28(\mathrm{~Hz})$. Same as the 2-m VAWTs, the first peak occurs at a frequency equal to the rotational frequency $(0.87 \mathrm{~Hz})$ of turbine.

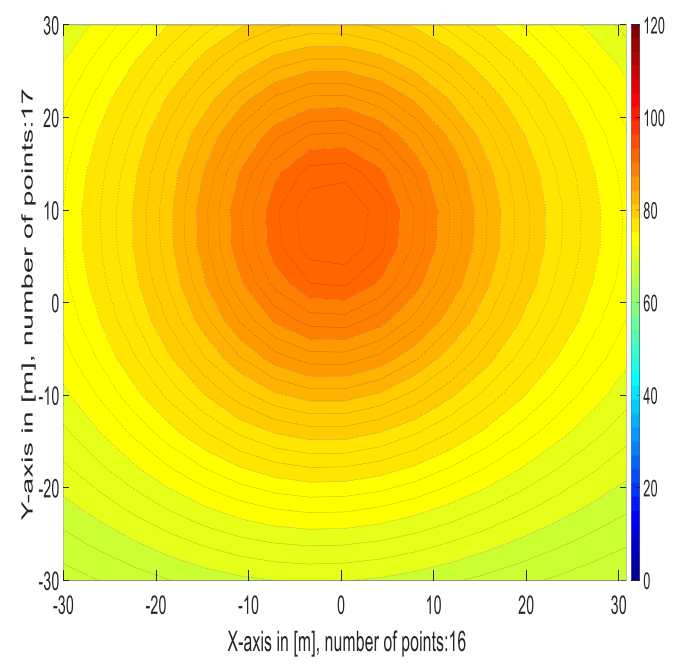

(a)

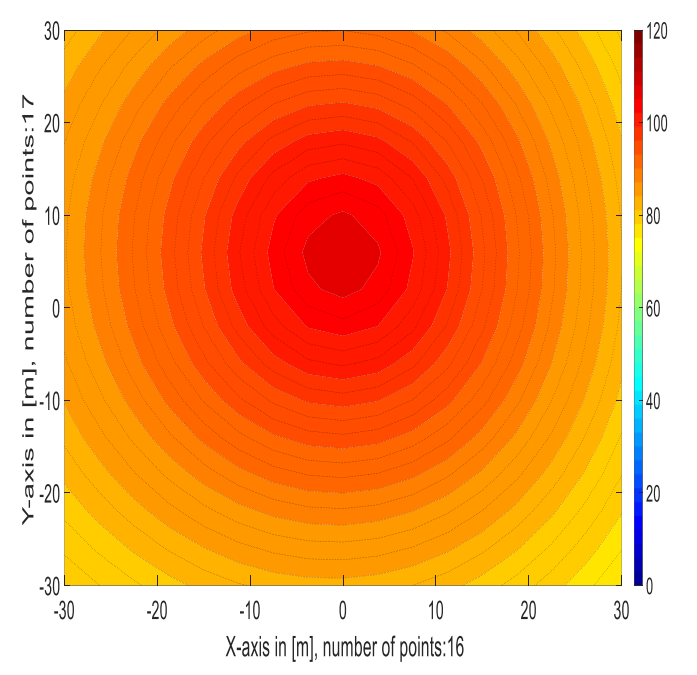

(b)

Figure 28 Acoustic Map at a Plane 2.5 $R_{t}$ Downstream from the Axis of the Turbine: a) 17-m Conventional VAWT and b) 17-m 50\% STS-VAWT. 


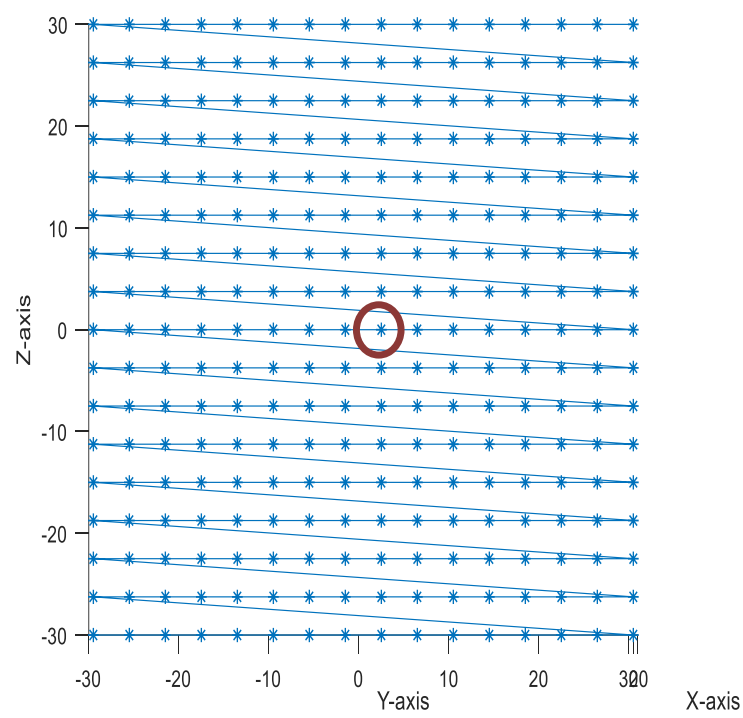

Figure 29 Exact Location of the Point that Acoustic Pressure for Different

Frequencies is Calculated on, in the 17-m VAWT Configurations.

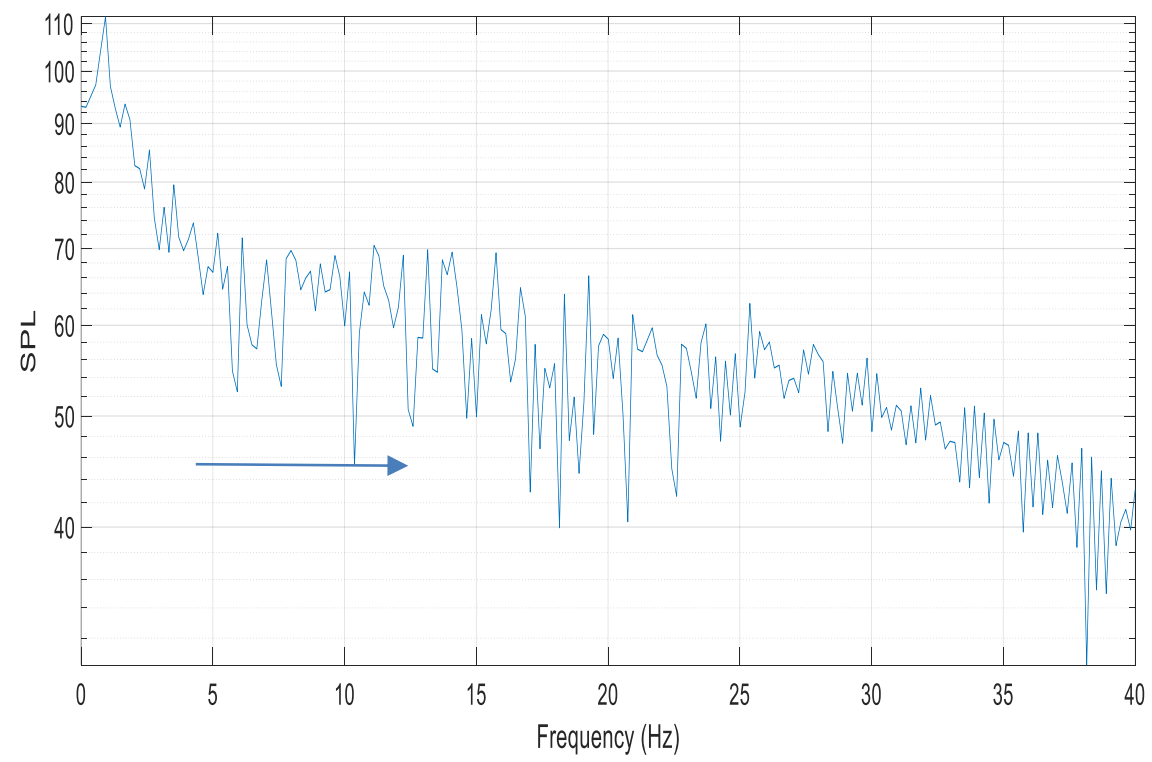

(a) 


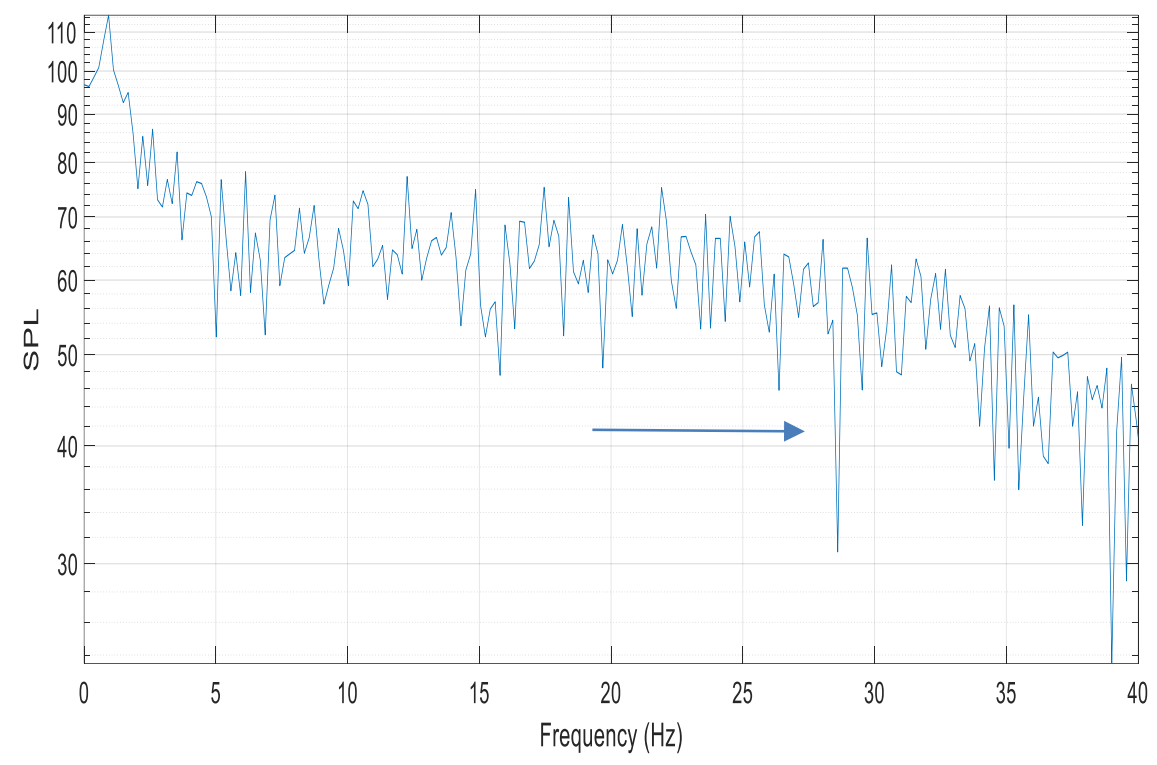

(b)

Figure 30 Acoustic Pressure vs. Frequency: a) 17m Conventional VAWT and b) 17m $50 \%$ STS-VAWT.

In the next study, the effect of $R_{t}$, on the acoustic intensity is evaluated. Five vertical planes are defined at different distances from the turbine's axis of rotation. Figure $\mathbf{3 1}$ is showing how these planes and turbine are placed within the domain. Two of planes are located at distances of $2.5 R_{t}$ and $5 R_{t}$ upstream of the turbine, and two other planes are located at the $2.5 R_{t}$ and $5 R_{t}$ downstream of turbine, while the last plane is at the turbine's axis of rotation. Characteristics of turbines used in these simulations are provided in the following table: 


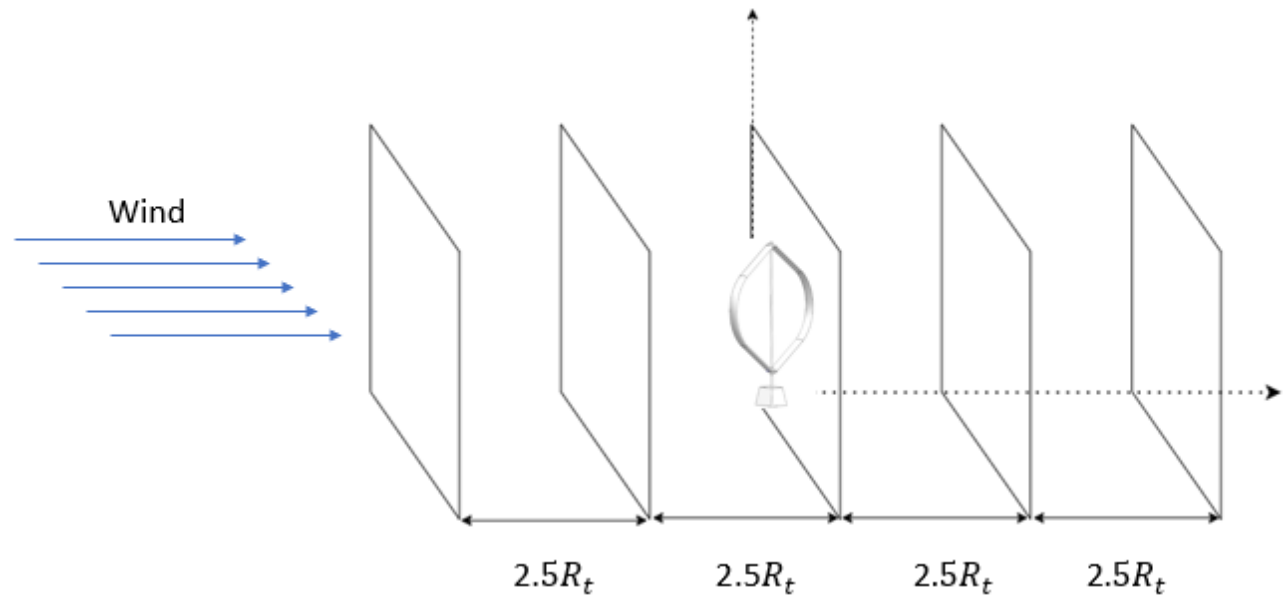

Figure 31 Schematic Showing Placement of Different Five Planes and the VAWT.

Table 6 Geometric Characteristics for Different VAWTs Configurations.

\begin{tabular}{|c|c|c|c|c|}
\hline Configuration & $\begin{array}{l}\text { 2-m } \\
\text { Conventional } \\
\text { VAWT }\end{array}$ & $\begin{array}{l}\text { 2-m 50\% } \\
\text { STS-VAWT }\end{array}$ & $\begin{array}{l}\text { 17-m } \\
\text { Conventional } \\
\text { VAWT }\end{array}$ & $\begin{array}{l}\text { 17-m 50\% } \\
\text { STS-VAWT }\end{array}$ \\
\hline Blade Shape & Troposkien & Troposkien & Troposkien & Troposkien \\
\hline Airfoil & NACA 0012 & NACA0012 & NACA 0015 & NACA0015 \\
\hline $\begin{array}{l}\text { Rotor Diameter } \\
\text { (m) }\end{array}$ & 0.979 & 0.979 & 16.732 & 16.732 \\
\hline Rotor Height (m) & 2 & 2 & 17 & 17 \\
\hline $\begin{array}{l}\text { Chord Length } \\
\text { (m) }\end{array}$ & 0.08815 & 0.10108 & 0.53 & 0.629 \\
\hline $\begin{array}{l}\text { Rotational Speed } \\
(\mathrm{rad} / \mathrm{s})\end{array}$ & 27.946 & 27.946 & 5.49 & 5.49 \\
\hline $\begin{array}{l}\text { Wind Speed } \\
(\mathrm{m} / \mathrm{s})\end{array}$ & 4.824 & 4.824 & 6.4443 & 6.4443 \\
\hline Tip Speed Ratio & 5.675 & 5.675 & 7.12 & 7.12 \\
\hline
\end{tabular}


The figure below demonstrates the acoustic prediction on the aforementioned planes for the 2-m conventional turbine.

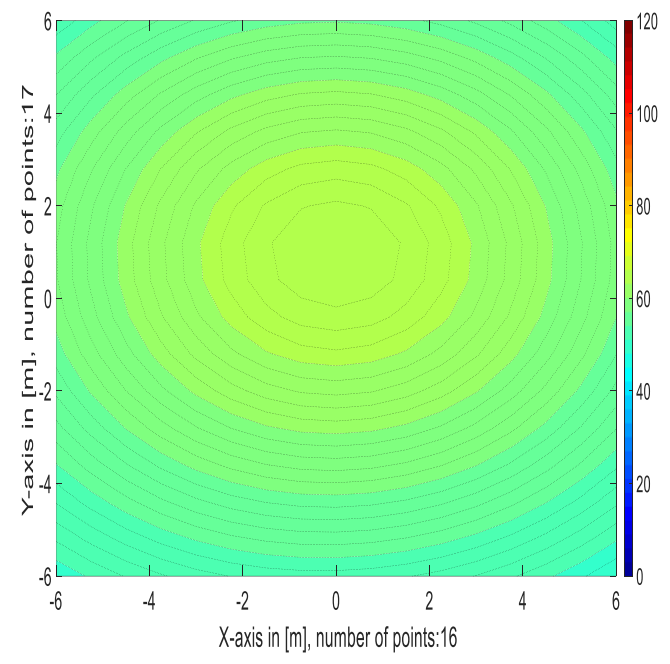

(a)

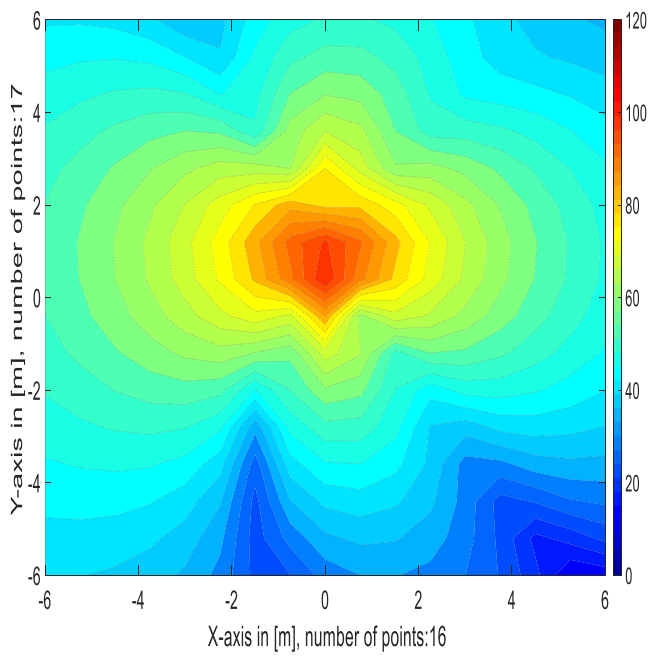

(c)

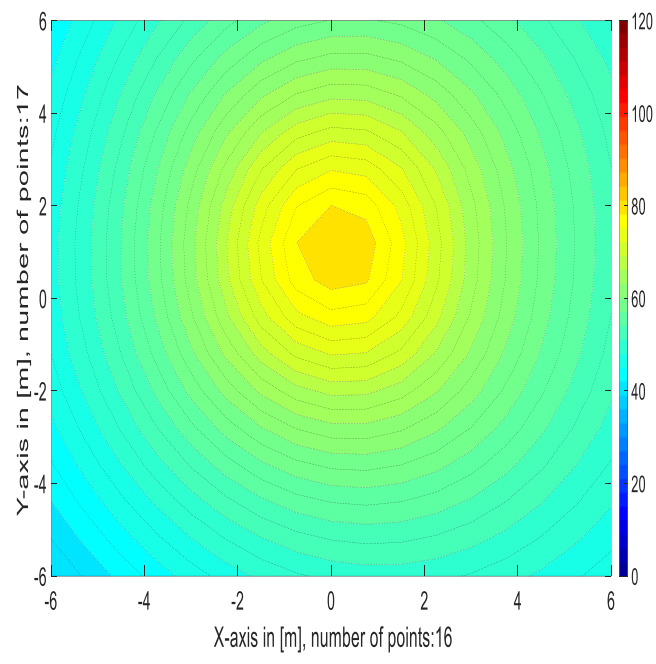

(b)

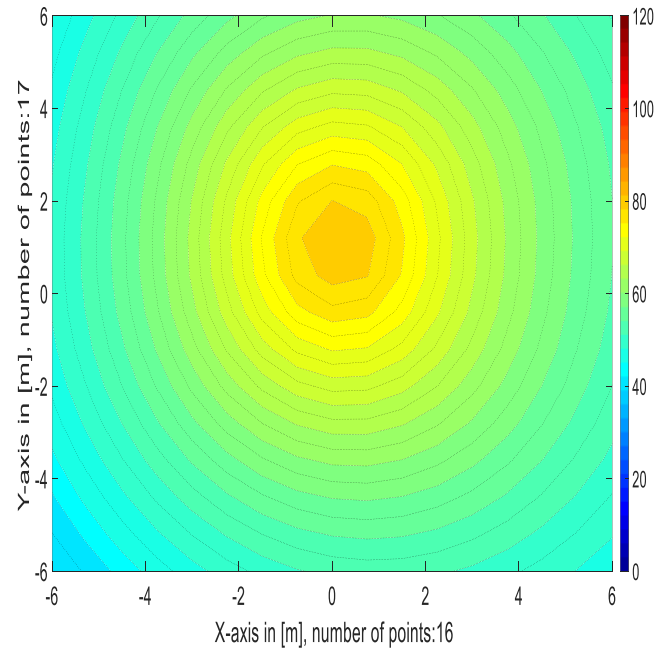

(d) 


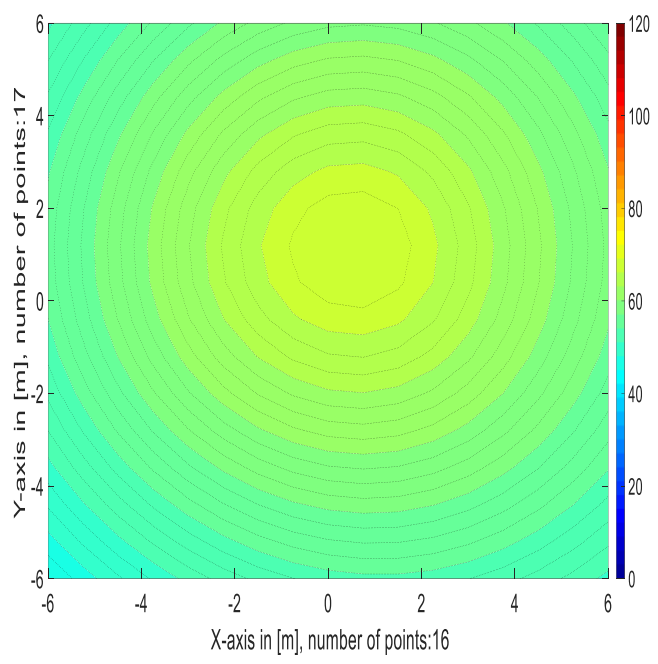

(e)

Figure 32 Acoustic Map Analysis on Different Planes for the 2-m Sandia Conventional Design. a) Plane Located at the $5 R_{t}$ Upstream, b) Plane Located at the 2.5 $R_{t}$ Upstream, c) Plane Located at the Turbine's Axis of Rotation, d) Plane Located at the 2.5 $R_{t}$ Downstream, e) Plane Located at the $5 R_{t}$ Downstream.

According to the figure above, noise intensity decreases as the distance from the turbine's axis of rotation increases, which is expected. On the planes that are located $5 R_{t}$ and $2.5 R_{t}$ upstream, maximum noise intensities are $65 \mathrm{~dB}$ and $75 \mathrm{~dB}$ respectively, while at the turbine's axis of rotation, maximum sound intensity equals to $105 \mathrm{~dB}$. Downstream, maximum noise intensities are $80 \mathrm{~dB}$ and $70 \mathrm{~dB}$ at the planes located $2.5 R_{t}$ and $5 R_{t}$, respectively. At the turbine's axis of rotation (Figure 32.c), the noise intensity is higher, and acoustic map looks different (with a "flower-like" shape) from the others. 


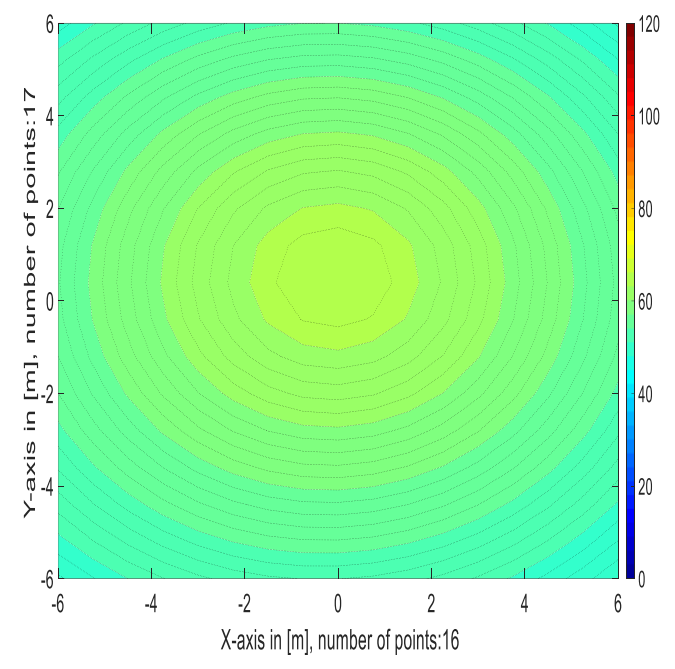

(a)

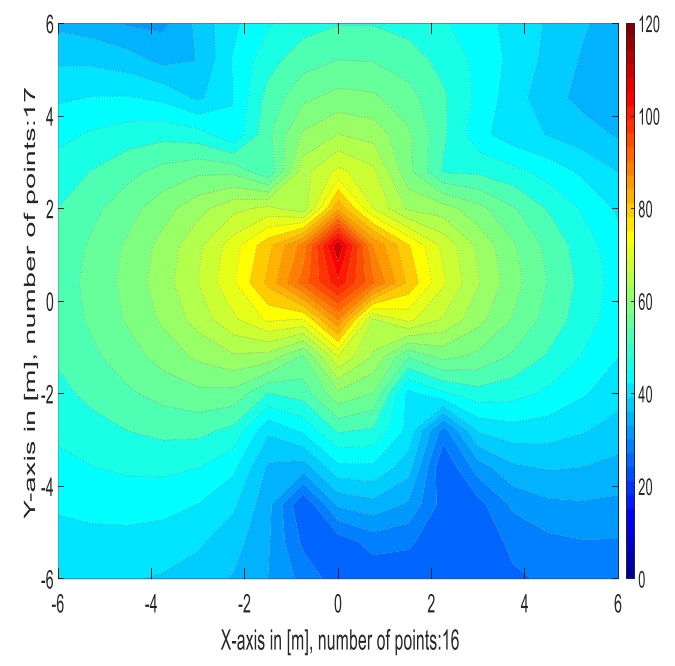

(c)

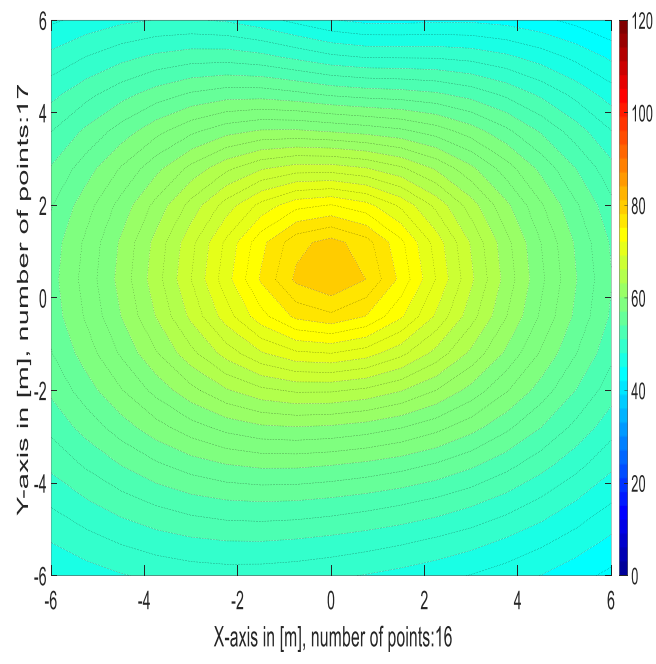

(b)

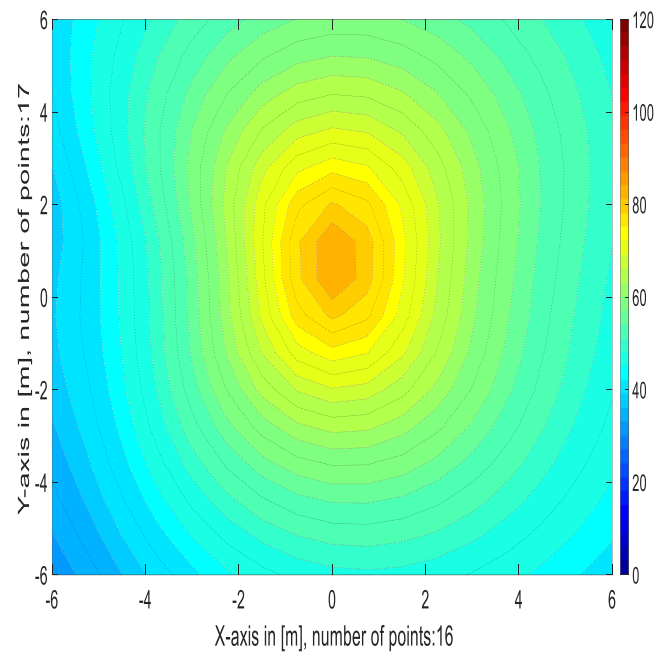

(d) 


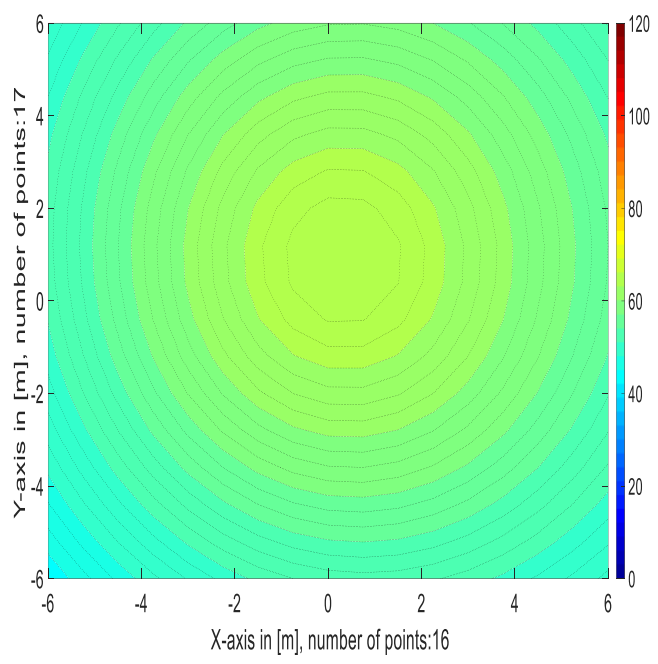

(e)

Figure 33 Acoustic Map Analysis on Different Planes for the 2-m 50\% STS Design. a) Plane Located at the $5 R_{t}$ Upstream, b) Plane Located at the $2.5 R_{t}$ Upstream, c) Plane Located at the Turbine's Axis of Rotation, d) Plane Located at the $2.5 R_{t}$ Downstream, e) Plane Located at the $5 R_{t}$ Downstream.

Figure 33 shows the acoustic map on the five different planes for the $2-\mathrm{m} 50 \%$ STSVAWT. On the planes which are located upstream, noise intensities are lower than the planes located after the turbine. Noise intensity increases by getting closer to the turbine's axis of rotation. Maximum values of noise intensity on the $5 R_{t}$ upstream, $2.5 R_{t}$ upstream, turbine's axis of rotation, $2.5 R_{t}$ downstream, and $5 R_{t}$ downstream are $65 \mathrm{~dB}, 80 \mathrm{~dB}, 110$ $\mathrm{dB}, 85 \mathrm{~dB}$ and $70 \mathrm{~dB}$, respectively.

Same study is performed on the 17-m Sandia Conventional VAWT, and results are provided in the following figure: 


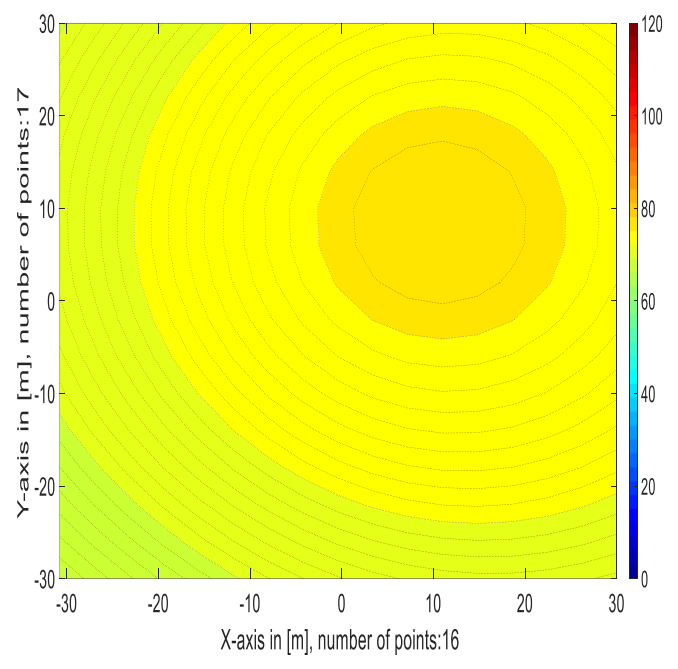

(a)

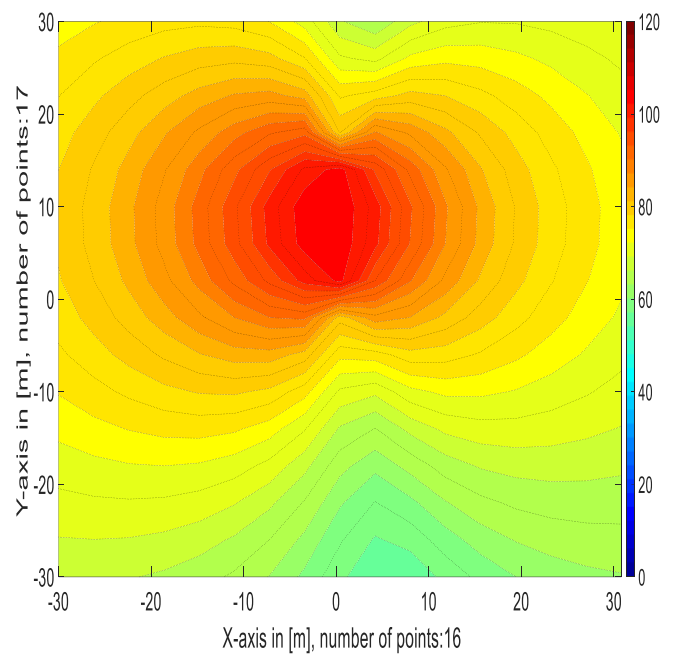

(c)

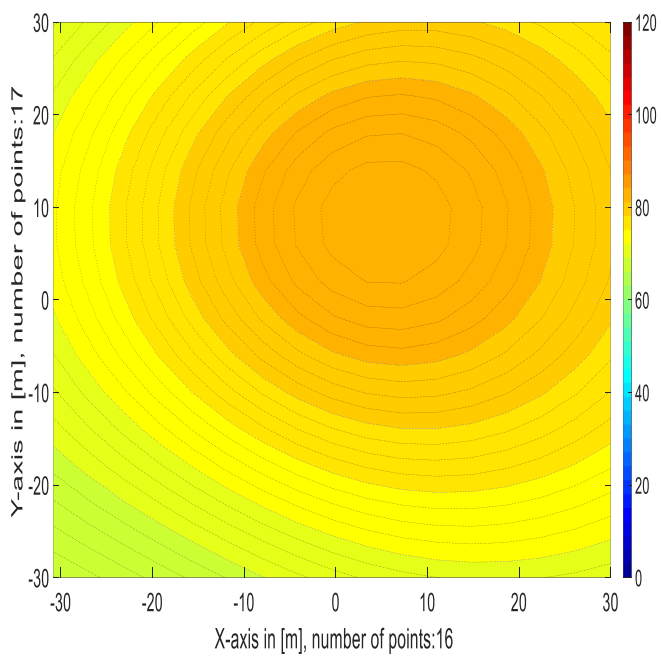

(b)

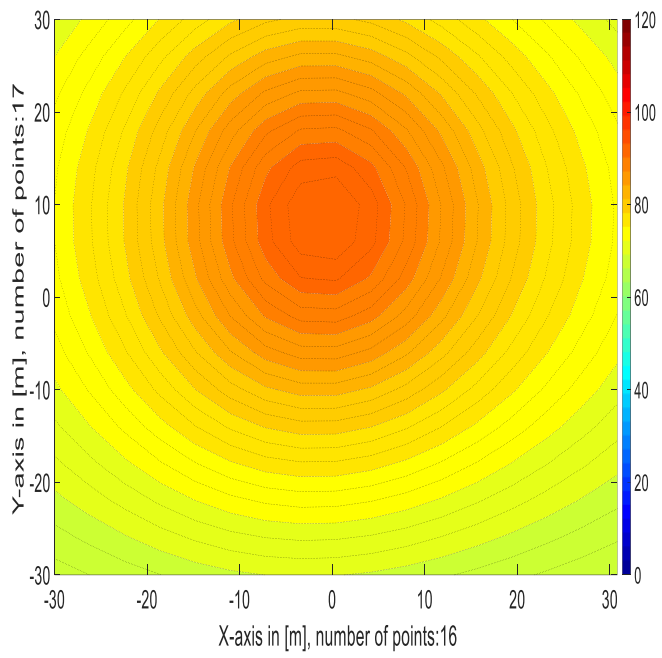

(d) 


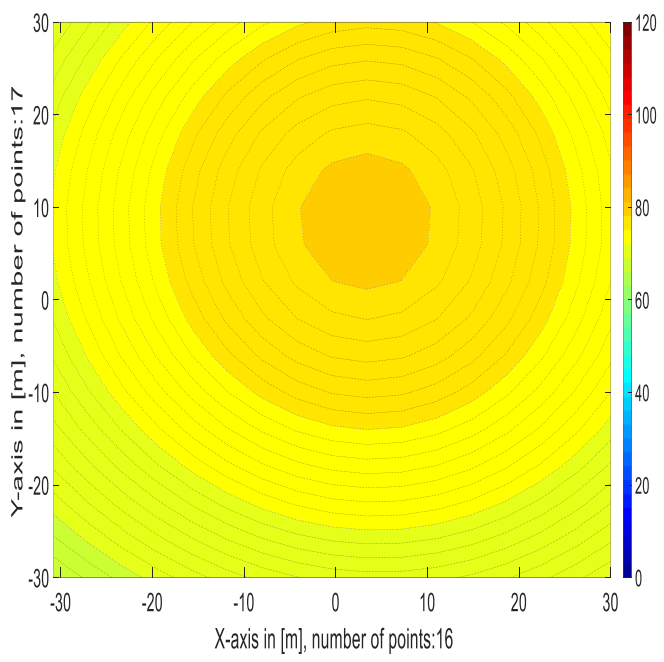

(e)

Figure 34 Acoustic Map Analysis on Different Planes for the 17-m Sandia Conventional Design. a) Plane Located at the $5 R_{t}$ Upstream, b) Plane Located at the 2.5 $R_{t}$ Upstream, c) Plane Located at the Turbine's Axis of Rotation, d) Plane Located at the 2.5 $R_{t}$ Downstream, e) Plane Located at the $5 R_{t}$ Downstream.

On the plane which is located $5 R_{t}$ before the turbine, noise intensity is $75 \mathrm{~dB}$ while on the plane that is located $2.5 R_{t}$ (also before the turbine), noise intensity increases ( $\left.85 \mathrm{~dB}\right)$. At the turbine's axis of rotation, acoustic map has a different "overlapping-circles" pattern (when compared to the "flower-like" pattern observed for the 2-m turbines) while having the highest noise intensity $(110 \mathrm{~dB})$. It is conjecture here that the non-symmetry in the "overlapping-circles" pattern may come from the rotation of the turbine (counter-clockwise from a bird's eye view). On the planes that are placed after the turbine, noise intensity values of $95 \mathrm{~dB}$ and $80 \mathrm{~dB}$ were obtained on the planes $2.5 R_{t}$ and $5 R_{t}$, respectively. 


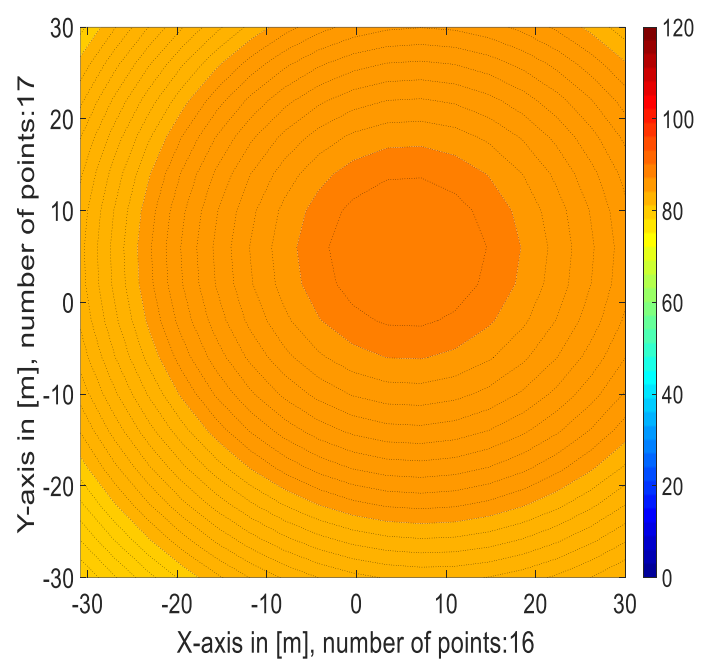

(a)

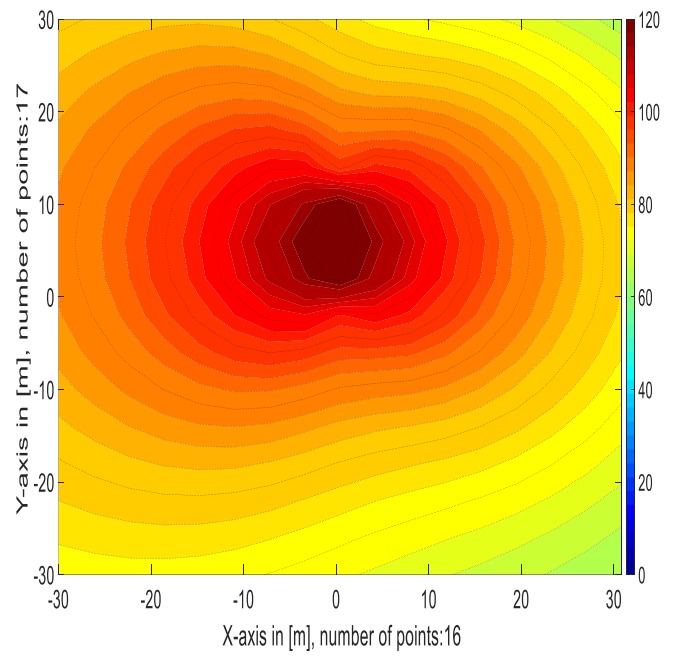

(c)

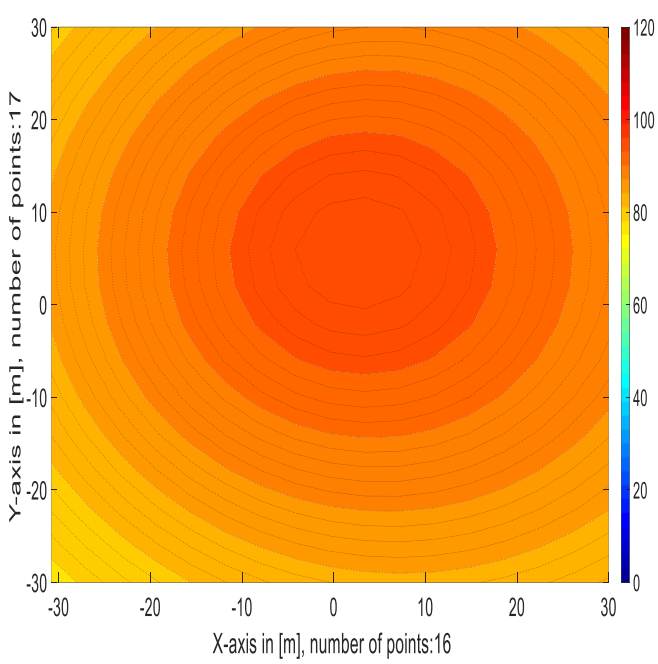

(b)

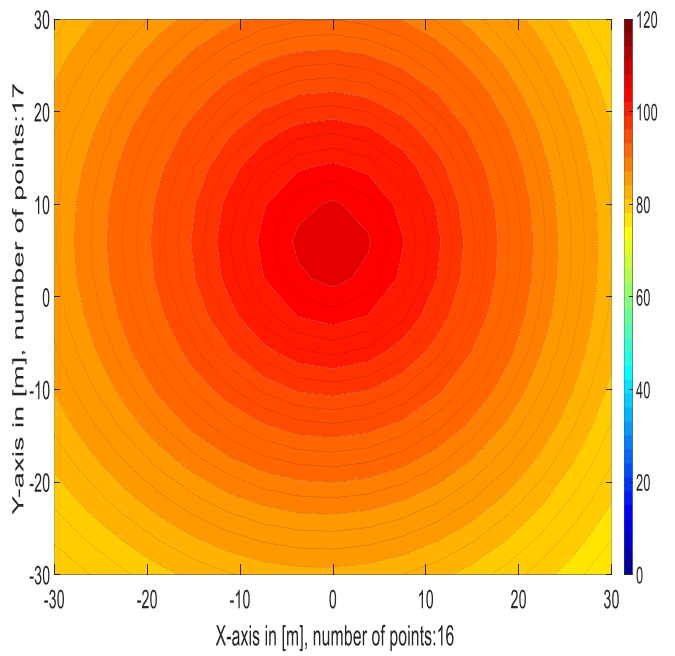

(d) 


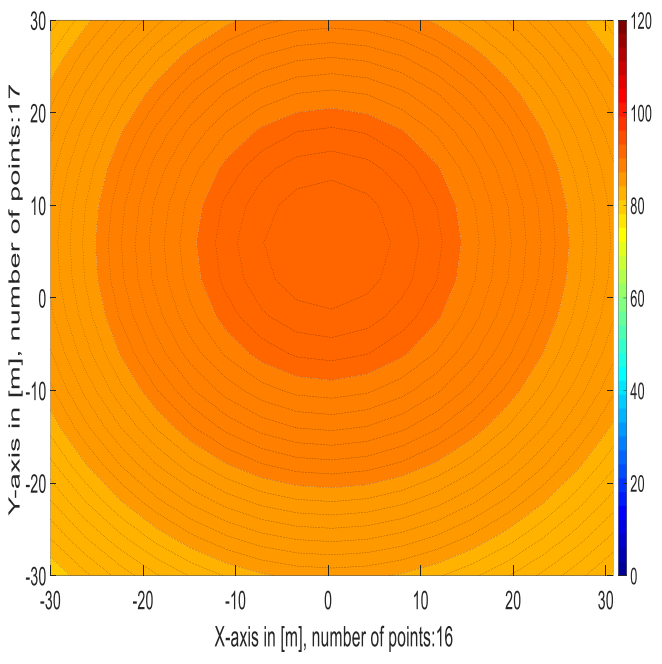

(e)

Figure 35 Acoustic Map Analysis on Different Planes for the 17-m 50\% STS Design. a) Plane Located at the $5 R_{t}$ Upstream, b) Plane Located at the $2.5 R_{t}$ Upstream, c) Plane Located at the Turbine's Axis of Rotation, d) Plane Located at the $2.5 R_{t}$ Downstream, e) Plane Located at the $5 R_{t}$ Downstream.

Acoustic maps on five different planes are depicted in the above figure for $17-\mathrm{m} 50 \%$ STSVAWT. As expected, noise intensity is higher on the planes close to the turbine's axis of rotation (on the $2.5 R_{t}$ upstream and downstream planes, maximum noise intensities are 95 $\mathrm{dB}$ and $105 \mathrm{~dB}$, respectively) and getting lower with increasing the distance (on the $5 R_{t}$ upstream and downstream maximum noise intensities equal $90 \mathrm{~dB}$ and $95 \mathrm{~dB}$ ). Despite having better aerodynamic performance, both 2-m and 17-m 50\% STS-VAWTs generate more acoustic noise when compared against conventional VAWTs at the locations simulated in the current investigation. 


\section{Chapter 5:}

\section{Conclusions, Contributions, and Recommendation for Future Work}

\subsection{Summary}

VAWTs have been less researched than HAWTs throughout the years, but there seems to be renewed interest these days due to offshore wind farm expansions towards deeper waters. In fact, VAWT can decrease turbine's manufacturing cost by as much as 20 percent.

Within the current work, the GENUVP code is used in the aerodynamic part of VAWT analysis. GENUVP is a code written in Fortran 90 and developed by the University of Athens which can predict the flow over multi-component configurations. This code uses a hybrid method which is a combination of unsteady panel and vortex particle wake methods. The concepts of these methods were explained in the second chapter. To manage the computational time and costs, GENUVP incorporates the sub-grid and particle mesh approximations.

The aeroacoustics solver used in the present work, was also developed by the University of Athens. Formulation 1C of the Ffowcs Williams - Hawkings equation for computing acoustic pressure generated by turbines is selected. Aeroacoustic code is using the thickness and loading sources which are calculated in the time using the panel method. Note that thickness and loading sources are affected by BWIs near the VAWT blades, hence the main contribution of the aeroacoustic analysis was to investigate the effect of BWI change, in the aeroacoustic analysis of the conventional VAWT and 50\% STS 
configurations. Aeroacoustics analysis was performed successfully for different VAWT sizes and configurations as discussed in Chapter 4.

\subsection{Conclusions}

Aerodynamic simulation results for both 2-m and 17-m conventional (Sandia) VAWTs showed relatively good agreement with available experimental data. According to the results obtained within this work, 50\% STS-VAWT has better performance than the conventional design. 50\% STS-VAWTs need less material in comparison to the conventional design.

In the $17-\mathrm{m}$ configurations, $50 \%$ STS-VAWT has a better performance than the conventional design for TSR $>6.2$, mainly due to: 1 ) The $50 \%$ STS model has a thicker chord compared to the conventional design and 2) The wake generated from the first plane interacts less with the wake generated by the second blade in the 50\% STS model [95]. 17m 50\% STS-VAWT has a lower manufacturing cost and similar aerodynamic performance when compared to the 17-m conventional design, becoming an alternative for future investigations and implementation.

Acoustic pressure around the turbine is calculated using a post processing code with the Formulation 1C of Ffowcs Williams - Hawkings equation. Considering the aeroacoustic results obtained within this work, noise intensity and total pressure values are in acceptable ranges. For the 2-m VAWT configurations, it can be concluded that conventional design generates less noise than the 50\% STS-VAWTs.

In 17-m configurations, conventional design generates the noise intensity of (95 dB) on a plane located $2.5 R_{t}$ off the turbine, while $50 \%$ STS-VAWT creates (105 dB). In 17 - 
$\mathrm{m}$ configurations, noise intensity is higher at the center of the plane where turbine is located and lower in the surroundings. Additional future investigations are needed to fully understand the increase in acoustic noise generated by $50 \%$ STS-VAWT when compared to the conventional VAWTs. Maximum noise intensities for the 2-m and 17-m configurations have an acceptable range. Although the noise intensity of $105 \mathrm{~dB}$ for the $50 \%$ STS model seems to be loud, but two points should be considered. First, $105 \mathrm{~dB}$ is calculated at the center of the plane while wind turbines are installed at a height from the ground, so the maximum noise intensity would be lower on the ground, second there are some regulations for the wind farms in which a minimum distance from the residential area should be considered to decrease the noise intensity to around 35 to $40 \mathrm{~dB}$, for example in Quebec a minimum distance of $750 \mathrm{~m}$ to $2 \mathrm{~km}$ from the residential area is considered. [97]

As expected, noise intensity increase by getting close to the turbine's axis of rotation and decrease by increasing the distance. On the planes located after the turbine "downstream planes", noise intensity is higher in comparison to upstream planes.

The usage of VAWTs can be a good alternative over the usage of the HAWT in the offshore deep-water power generation. HAWTs on floating platforms make the turbine unstable because the heavy parts of the turbine (nacelle and generator) are mounted higher in the turbine. Considering the results gained from this work, it can be suggested that the $50 \%$ STS model can become an alternative for offshore deep-water power generation. Further experimental analysis should be investigated. 


\subsection{Recommendations}

- Aerodynamic and aeroacoustics experimental tests of 2-m 50\% STS-VAWTs are recommended to provide further validation data for the current work.

- It would be interesting to explore small wind farm configurations with 3 to $450 \%$ STS-VAWTs and compare against conventional VAWTs.

- Simulations can be improved by adding a non-lifting tower, an atmospheric boundary layer, acoustic attenuation surfaces, etc.

- Aeroelastic behavior of the turbines can also be explored in the future, mainly for the shifted design. 


\section{References}

[1] M. Bhutta, N. Hayat, A. Farooq, U. Ahmed, Z. Ali, S. Jamil, Z. Hussain, "Vertical Axis Wind Turbine - A Review of Various Configurations and Design

Techniques," Renewable and Sustainable Energy Reviews, vol. 16, pp. 1926-1939, 2012.

[2] J. Baker, "Features to Aid or Enable Self Starting of Fixed Pitch Low Solidity Vertical Axis Wind Turbines," Journal of Wind Engineering and Industrial Aerodynamics, vol. 15, pp. 369-380, 1983.

[3] M. Hansen, "Aerodynamics of Wind Turbines," Second Edition, Routledge, 2015.

[4] "Global Offshore Wind Report," Global Wind Energy Council "https://gwec.net/", 2020.

[5] I. Paraschivoiu, "Wind Turbine Design with Emphasis on Darrieus Concept," Presses Inter Polytechnique, 2002.

[6] D.A. Spera, "Wind Turbine Technology: Fundamental Concepts of Wind Turbine Engineering," ASME Press New York, 1994.

[7] H.J. Sutherland, D.E. Berg, T.D. Ashwill, "A Retrospective of VAWT Technology," Sandia National Laboratories, pp. 1-64, 2012.

[8] M.M. Aslam Bhutta, N.Hayat, A.U. Farooq, Z. Ali, S.R. Jamil, Z. Hussain, "Vertical Axis Wind Turbine: A Review of Various Configurations and Design Techniques," Renewable and Sustainable Energy Reviews, vol. 16, pp. 1926-1939, 2012. 
[9] D. Hilewit, E. Matida, A. Fereidooni, H. Abo El Ella, F. Nitzsche, "Power Coefficient Measurements of a Novel Vertical Axis Wind Turbine," Energy Science \& Engineering, vol. 7, pp. 2373-2382, 2019.

[10] S. Oerlemans, P. Sijtsma, B. Méndez López, "Location and Quantification of Noise Sources on a Wind Turbine," Journal of Sound and Vibration, vol. 299, pp. 869883, 2007.

[11] K.P. Waye, E. Ohrstrom, "Psycho-Acoustic Characters of Relevance for Annoyance of Wind Turbine Noise," Journal of Sound and Vibration, vol. 250, pp. 65-73, Feb. 2002.

[12] A.J. Bedard, T.M. Georges, "Atmospheric Infrasound," Acoustics Australia, vol. 28, pp. 47-52, 2000.

[13] J. Delfs, "Basics of Aeroacoustics," Lecture Notes. Braunschweig, Germany: DLRGerman Aerospace Center, 2008.

[14] M. Howe, "Acoustics of Fluid-Structure Interations," Cambridge: Cambridge University Press, 1998.

[15] H. Glauert, "Air Plane Propellers," in Aerodynamic Theory, Springer, pp. 169-360, 1935.

[16] J.F Manwell, J.G. Mc Gowan , A.L. Rogers, "Wind Energy Explained :Theory, Design and Application," John Wiley \& Sons, 2010.

[17] X. Liu, L. Wang, X. Tang, "Optimized Linearization of Chord and Twist Angle Profiles for Fixed-Pitch Fixed-Speed Wind Turbine Blades," Journal of Renewable Energy, vol. 57, pp. 111-119, 2013. 
[18] O. Vries, "Fluid Dynamic Aspects of Wind Energy Conversion," Advisory Group for Aerospace Research and Development Neuilly-Sur-Seine (France), 1979.

[19] G. Xu , L.N. Sankar, "Application of a Viscous Flow Methodology to the NREL Phase VI Rotor," in Wind Energy Symposium, pp. 83-93, 2002.

[20] M.M. Hand , D. Simms , L. Fingersh , D. Jager , J. Cotrell, "Unsteady Aerodynamics Experiment Phase V: Test Configuration and Available Data Campaigns," National Renewable Energy Lab., Golden, CO (US), 2001.

[21] P.J. Moriarty, A.C. Hansen, "AeroDyn Theory Manual," National Renewable Energy Lab, Colorado, USA, 2005.

[22] E. Bossanyi, "GH Bladed-Theory Manual," Garrad Hassan and Partners, vol. 16, 2008.

[23] L. Marshall, J. Buhl Junior, "A New Empirical Relationship Between Thrust Cefficient and Induction Factor for the Turbulent Windmill State," National Renewable Energy Laboratory, Golden, CO, United States, 2005.

[24] H. Glauert, "The Analysis of Experimental Results in the Windmill Brake and Vortex Ring States of an Air Screw," HM Stationery Office, 1926.

[25] T. Burton, D. Sharpe , N. Jenkins , E. Bossanyi, "Handbook of Wind Energy," John Wiley \& Sons, 2001.

[26] R.B. Noll, N.D. Ham, "Effects of Dynamic Stall on SWECS [Small Wind Energy Conversion Systems]," Journal of Solar Energy Engineering, pp. 96-101, 1982.

[27] H. Abramovich, "Vertical Axis Wind Turbines: a Survey and Bibliography," Wind Engineering, vol. 11, pp. 334-343, 1987. 
[28] C. Tran, D. Petot, "Semi-Empirical Model for the Dynamic Stall of Airfoils in View of the Application to the Calculation of Responses of a Helicopter Blade in Forward Flight," 1980.

[29] F. Tarzanin, "Prediction of Control Loads due to Blade Stall," Journal of American Helicopter Society, vol. 17, pp. 33-46, 1972.

[30] H. Snel, "Heuristic Modelling of Dynamic Stall Characteristics," in EWECConferance, Bookshop for Scientific Publications, pp. 429-433, 1997.

[31] J. Leishman, T. Beddoes, "A Semi-Empirical Model for Dynamic Stall," Journal of American Helicopter Society, vol. 34, pp. 3-17, 1989.

[32] M.H. Hansen, M. Gaunaa, H.A. Madsen, "A Beddoes-Leishman Type Dynamic Stall Model in State-Space and Indicial Formulations," 2004.

[33] H. Bijl , A.H. Van Zuijlen, A. Van Mameren, "A Validation of Adaptive Unstructured Hexahedral Mesh Computations of Flow Around a Wind Turbine Airfoil," International Journal for Numerical Methods Fluids, vol. 48, pp. 929-945, 2005.

[34] A. Van Zuijlen, H. Bijl, G. Dufour, A. Van Mameren, "Evaluation of Adaptive, Unstructured CFD Calculations of the Flow Around The DU 91 Airfoil," in European Wind Energy Conference \& Exhibtion, Madrid, pp. 1-5, 2003.

[35] T.J. Carrigan, B.H. Dennis , Z.X. Han, B.P. Wang, "Aerodynamic Shape Optimization of a Vertical Axis Wind Turbine Using Differential Evolution," International Scholarly Research Notices Renewable Energy, vol. 2012, 2012.

[36] B. Sanderse, "Aerodynamics of Wind Turbine Wakes," Petten: ECN, 2009. 
[37] F. Menter, "Improved Two-Equation k-omega Turbulence Models for Aerodynamic Flows," Nasa Technical Report , vol. 93, pp. 22809, 1992.

[38] C.K.G. Lam, K. Bremhorst, "A Modified Form of the k- $\varepsilon$ Model for Predicting Wall Turbulence," Journal of Fluids Engineering, vol. 103, pp. 456-460, 1981.

[39] S. Kalvig, E. Manger, B. Hjertager, "Comparing Different CFD Wind Turbine Modelling Approaches with Wind Tunnel Measurement," in Journal of Physics: Conference Series, IOP Publishing, pp. 12056, 2014.

[40] S.C. Cakmakcioglu, O. Tugluk, I.O. Sert, N.S. Uzol, "2-D and 3-D CFD Investigation of NREL S826 Airfoil at Low Reynolds Numbers," in Journal of Physics: Conference Series, IOP Publishing, pp. 12028, 2014.

[41] W.Z. Shen, W.J. Zhu, J.N. Sørensen, "Study of Tip Loss Corrections Using CFD Rotor Computations," in Journal of Physics: Conference Series, IOP Publishing, pp. 12094, 2014.

[42] W. Rankine, "On the Mechanical Principles of The Action of Propellers," Transactions of the Institution of Naval Architects, vol. 6, 1865.

[43] R. Froude, "On The Part Played in Propulsion by Differences of Fuid Pressure," Transactions of the Institution of Naval Architects, vol. 30, pp. 390, 1889.

[44] J.N. Sørensen ,W.Z. Shen, "Numerical Modeling of Wind Turbine Wakes," Journal of Fluid Engineering, vol. 124, pp. 393-399, 2002.

[45] X. Shen, J.G. Chen, X.C. Zhu, Z.H. Du, "Multi-Objective Optimization of Wind Turbine Blades Using Lifting Surface Method," Journal of Energy, vol. 90, pp. 1111-1121, 2015. 
[46] M.A. Kotb, M.M. Abdel Haq, "A Rigid Wake Model for a Horizontal Axis Wind Turbine," Wind Engineering, vol. 16, no. 2, pp. 95-108, 1992.

[47] F.N. Coton, T.G. Wang, R.A.M. Galbraith, "An Examination of Key Aerodynamic Modelling Issues Raised by The NREL Blind Comparison," Wind Energy: an International Journal for Progress and Applications in Wind Power Conversion Technology, vol. 5, no. 2-3, pp. 199-212, 2002.

[48] F.N. Coton, T.G. Wang, "The Prediction of Horizontal Axis Wind Turbine Performance in Yawed Flow Using an Unsteady Prescribed Wake Model," Journal of Power and Energy, vol. 213, pp. 33-43, 1999.

[49] T.G. Wang, F.N. Coton, "An Investigation of The Effect of Tip Vortex Mass Injection of Blade Vortex Interaction," Journal of Wind Engineering, vol. 89, pp. $873,2001$.

[50] T. Wang, L. Wang, W. Zhong, "Large-Scale Wind Turbine Blade Design and Aerodynamic Analysis," Chinese Science Bulletin, vol. 57, pp. 466-472, 2012.

[51] M. Døssing, "Vortex Lattice Modelling of Winglets on Wind Turbine Blades," Risø National Laboratory, Technical University of Denmark, 2007.

[52] S. Chkir, "Unsteady Loads Evaluation for a Wind Turbine Rotor Using Free Wake Method," Energy Procedia, vol. 6, pp. 777-785, 2011.

[53] L.A. Viterna, D.C. Janetzke, "Theoretical and Experimental Power from Large Horizontal-Axis Wind Turbines," NASA-TM-82944, Cleveland, USA, 1982. 
[54] C. Lindenburg, "Stall Coefficients, Aerodynamic Airfoil Coefficient at Large Angles of Attack," National Renewable Energy Laboratory, Golden, Colorado, USA, 2001.

[55] F. Richez, I. Mary, V. Gleize, C. Basdevant, "Zonal RANS/LES Coupling Simulation of a Transitional and Separated Flow, Around an Airfoil, Near Stall," Theoretical and Computational Fluid Dynamics, vol. 22, pp. 305-315, 2008.

[56] S. Schmidt , F. Thiele, "Detached Eddy Simulation of Flow Around an-Airfoil," Flow, Turbulence and Combustion, vol. 71, pp. 261, 2003.

[57] S. Eisenbach, R. Friedrich, "Large-Eddy Simulation of Flow Separation on an Airfoil at a High Angle of Attack and Re = 105, Using Cartesian Grids," Theoretical and Computational Fluid Dynamics, vol. 22, pp. 213-225, 2008.

[58] M.R. Visbal, R.E. Gordnier, M.C. Galbraith, "High-Fidelity Simulations of Moving and Flexible Airfoils at Low Reynolds Numbers," Experiments in Fluids, vol. 46, pp. 903, 2009.

[59] M. Lighthill, "On Sound Generated Aerodynamically I. General theory," Proceedings of The Royal Society of London. Series A. Mathematical and Physical Sciences, vol. 211, pp. 546-587, 1952.

[60] N. Curle, "The Influence of Solid Boundaries Upon Aerodynamic Sound," Proceedings of the Royal Society of London. Series A. Mathematical And Physical Sciences, vol. 231, pp. 505-514, 1955. 
[61] K.S. Brentner, F. Farassat, "An Analytical Comparison of The Acoustic Analogy and Kirchhoff Formulation for Moving Surfaces," AIAA Journal, vol. 36, pp. 1379-1386, 1998.

[62] K. Brentner, "Modeling Aerodynamically Generated Sound : Recent Advances in Rotor Noise Prediction," in 38th Aerospace Sciences Meeting and Exhibit, pp. 345, 2000.

[63] A. Najafi-Yazdi, G.A. Bres, L. Mongeau, "An Acoustic Analogy Formulation for Moving Sources in Uniformly Moving Media," Proceedings of The Royal Society Mathematical Physical and Engineering Sciences, vol. 467, pp. 144-165, 2010.

[64] K.S. Brentner, F. Farassat, "Modeling Aerodynamically Generated Sound of Helicopter Rotors," Progress in Aerospace Sciences, vol. 39, pp. 83-120, Feb. 2003.

[65] T.F. Brooks, D.S. Pope, M.A. Marcolini, "Airfoil Self-Noise and Prediction," National Aeronautics and Space Administration, Office of Management, vol 1218, 1989.

[66] N.D. Kelley, R.R. Hemphill, D.L. Sengupta, "Television Interference and Acoustic Emissions Associated with the Operation of the Darrieus VAWT," in Proceedings of the 5th Biennial Wind Energy Conference and Workshop, pp. 397-413, 1981.

[67] C.E. Pearson, W. Graham, "Investigation of The Noise Sources on a Vertical Axis Wind Turbine Using an Acoustic Array," in 19th AIAA/CEAS Aeroacoustics Conference, pp.1-10, 2013. 
[68] C. Pearson, "Vertical Axis Wind Turbine Acoustics," Phd, Cambridge University, 2013.

[69] W. Johnson, "Rotorcraft Aerodynamics Models for a Comprehensive Analysis," in Annual Forum Proceedings-American Helicopter Society, pp. 71-94, Washington, DC, 1998.

[70] D.A. Wachspress, T.R. Quackenbush, A.H. Boschitsch, "Rotorcraft Interactional Aerodynamics Calculations with Fast Vortex / Fast Panel Methods," in Annual Forum Proceedings American Helicopter Society, Virginia Beach, VA, Proc. American Helicopter Society 56th Annual Forum, pp. 51-71, 2000.

[71] J. Leishman, "Principles of Helicopter Aerodynamics," Cambridge University Press, New York, 2000.

[72] S.G. Voutsinas, "A GENeralized Unsteady Vortex Particle Method for Solving the Unsteady Flow Around Multi-Component Configurations, Fluids Section," Department of Mechanical Engineering, National Technical University of Athens, Greece, 1990.

[73] S.G. Voutsinas, M.A. Belessis, K.G. Rados, "Investigation of The Yawed Operation of Wind Turbines By Means of a Vortex Particle Method," Proc. FDP Symposium on Aerodynamics and Aeroacoustics of Rotorcraft (AGARD CP552), Berlin, Germany, 1995.

[74] S.G. Voutsinas, D.G. Triantos, "High-Resolution Aerodynamic Analysis of Full Helicopter Configurations," Proc. 25th European Rotorcraft Forum, Rome, Italy, Sept. 14-16, 1999. 
[75] S.G. Voutsinas, D.G. Triantos, "Aeroacoustics of Full Helicopter Configurations Using Vortex Particle Flow Approximations," Proc. CEAS Forum on Aeroacoustics of Rotors and Propellers, Rome, Italy, June 9-11, 1999.

[76] J.L. Hess, A.M.O. Smith, "Calculation of Non-Lifting Potential Flow about Arbitrary Three-Dimensional Bodies," McDonnell Douglas Tech. Report ES40622.

[77] J. Hess, "Calculation of Potential Flow about Arbitrary Three-Dimensional Lifting Bodies," McDonnell Douglas Tech. Report MDCJ5679-01, 1972.

[78] C. Rehbach, "Calcul d'Ecoulements Autour Dailes Sans Epaisseur Avec Nappes Tourbillonnaires Evolutives," Recherche Aerospatiale, vol. 2, pp. 53-61, 1973.

[79] A. Majda, J.T. Beale, "Vortex Methods I: Convergence in Three Dimensions," Mathematics of Computation, vol. 39, pp. 1-27, July 1982.

[80] J.T. Beale, A. Majda, "High Order Accurate Vortex Methods with Explicit Velocity Kernels," Journal of Computational Physics, vol. 58, pp. 188-208, 1985.

[81] D.G.Triantos, "Aerodynamic and Aeroacoustic Analysis of Helicopter Rotors, PhD Dissertation, Fluids Section," Dept. of Mechanical Engineering, National Technical University of Athens, Greece, 2002.

[82] J.C. Vassberg, "A Fast Surface Panel Method Capable of Solving Million-Element Problems," in 35th Aerospace Sciences Meeting and Exhibit, pp. 97-168, 1997.

[83] R.W. Hockney, J.W. Eastwood, "Computer Simulation Using Particles," New York: McGraw-Hill, 1981. 
[84] C. Anderson, "A Method of Local Corrections for Computing the Velocity Due to a Distribution of Vortex Blobs," Journal of Computational Physics, vol. 62, pp. 111$123,1986$.

[85] B. Blackwell, G. Reis, "Blade Shape for a Tropskien Type of Vertical-Axis Wind Turbine," Sandi Labs, Albuquerque, 1977.

[86] K. Truong, "Modeling Aerodynamics, Including Dynamic Stall, for Comprehensive Analysis of Helicopter Rotors," Journal of Aerospace, vol. 4, pp. 21, 2017.

[87] B.F. Blackwell, R.E. Sheldahl, "Selected Wind Tunnel Test Results for the Darrieus Wind Turbine," Journal of Energy, vol. 1, pp. 382-386, 1997.

[88] M.H. Worstell, "Aerodynamic Performance of the 17 Meter Diameter Darrieus Wind Turbine," NASA STI Recon Technical Report, vol. 79, pp. $27670,1979$.

[89] A. Tadamasa, M. Zangeneh, "Numerical Prediction of Wind Turbine Noise," Renewable Energy, vol. 36, pp. 1902-1912, July 2011.

[90] D. Casalino, "An Advanced Time Approach for Acoustic Analogy Predictions," Journal of Sound and Vibration, vol. 261, pp. 583-612, 2003.

[91] F. Farassat, "Derivation of Formulations 1 and 1A of Farassat," NASA Langley Research Center, Hampton, Virginia, 2007.

[92] H. Kuttruff, "Acoustics: an Introduction," Abingdon: Taylor and Francis, 2007.

[93] M. Pidd, A. Dummel, D. Falchero, M. Genito , J. Prospathopoulos, A. Visingardi, S. Voutsinas, J. Yin, "Validation of Aeroacoustic Predictions Using the Helinovi Database," in 32nd European Rotorcraft Forum, Maastricht, Netherlands, 2006. 
[94] T. Andronikos, G. Papadakis, V.A. Riziotis, "Aerodynamic Analysis of Helicopter in Interaction with Wind Turbine's Wake, " 2018.

[95] D. Hilewit, E. Matida, A. Fereidooni, H. Abo El Ella, F. Nitzsche, "Numerical Investigations of a Novel Vertical Axis Wind Turbine," Energy Science \& Engineering, vol. 7, pp. 2498-2509, 2019.

[96] J.E. Ffowcs Williams, D.L. Hawkings, "Sound Generation by Turbulence and Surfaces in Arbitrary Motion," Philosophical Transactions of the Royal Society of London. Series A, Mathematical and Physical Sciences, vol. 264, pp. 321-342, 1969.

[97] https://ontario-wind-resistance.org/setbacks/

\section{Appendices}

\section{Appendix A GENUVP Code}


In the current appendix, some extra information, and features about the GENUVP code which is used in this Thesis is discussed. Discussion includes the inputs that the code use and a precise flowchart about code's operation.

\section{A.1 Input Files}

Conditions and configurations are defined by the GENUVP using four different input files. They are dfile,geo,geo and .cld.

dfile: this file is an input of general data that used to define the configuration. dfile contains the following:

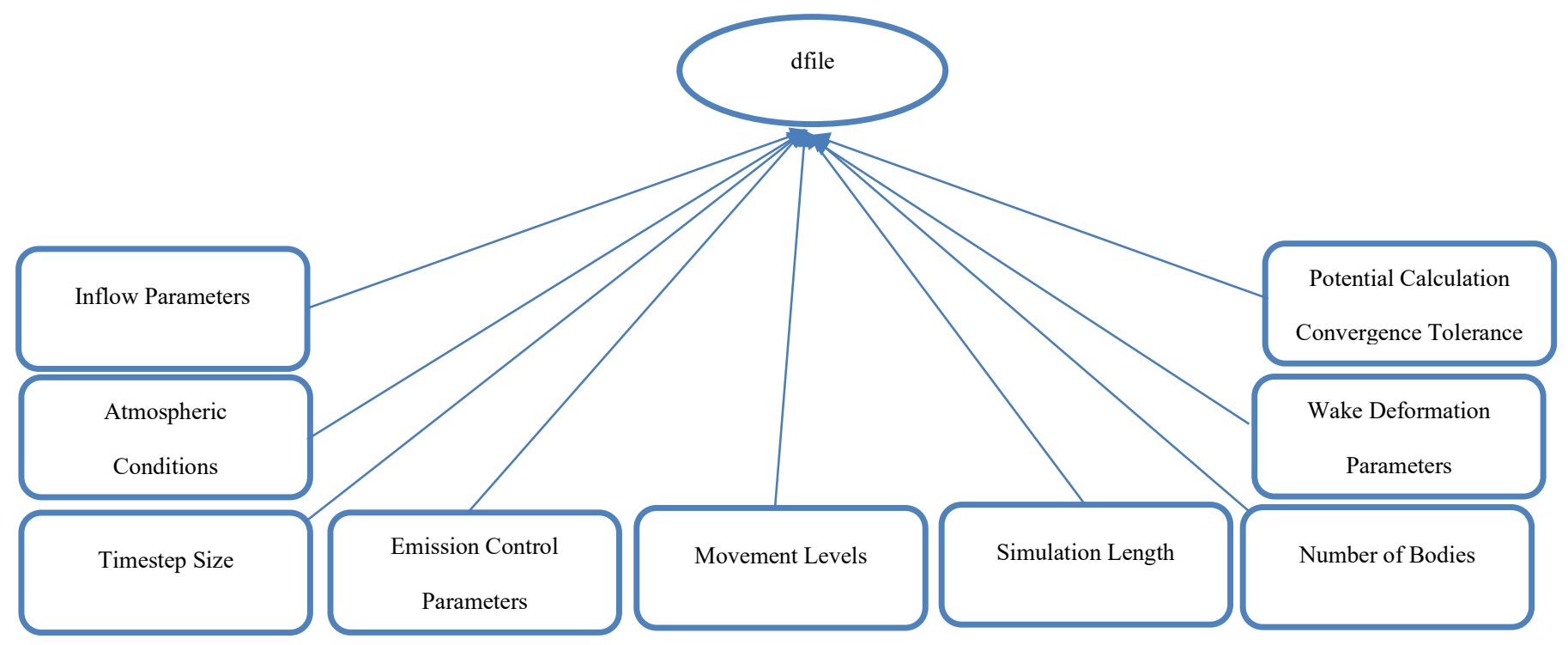

.geo: this file is used primarily to define the body movements and includes followings:

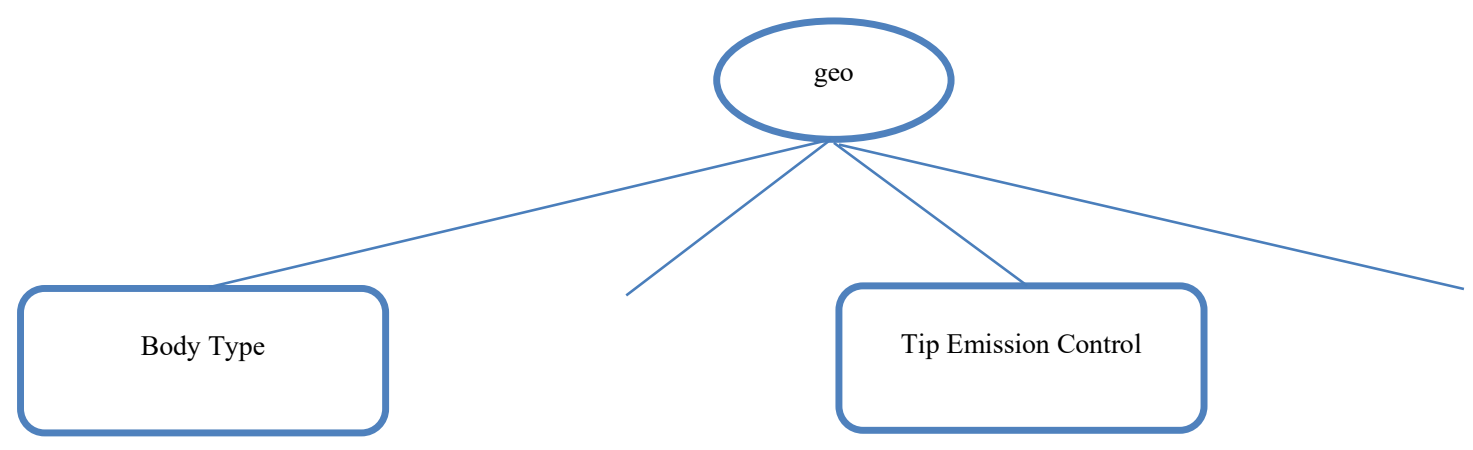


geo: this file is used to define the body geometry and contains the followings:

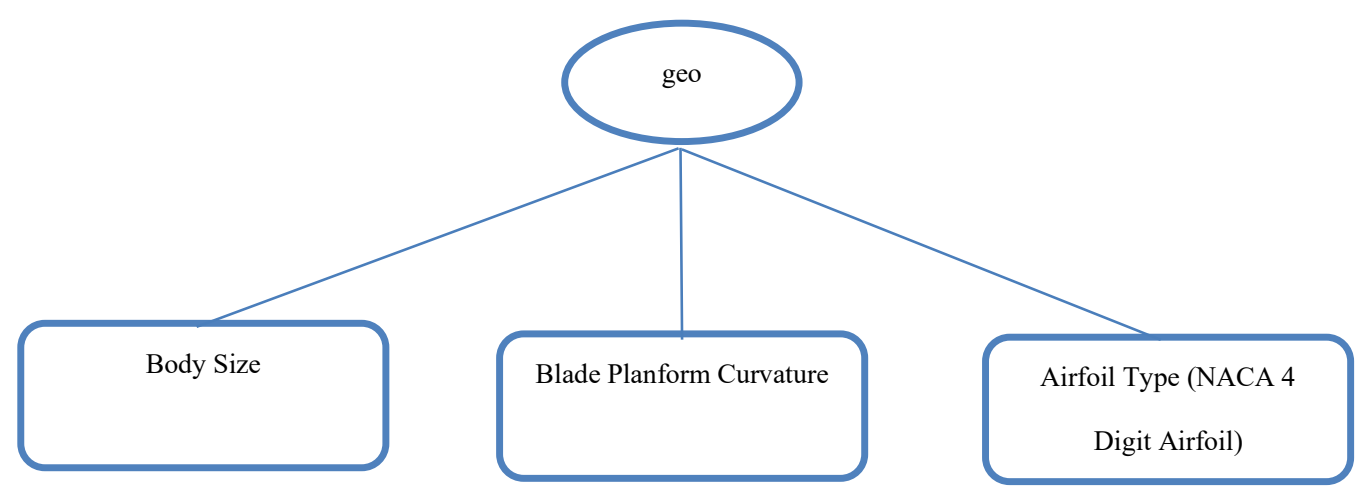

cld: this file contains 2D Airfoil information to correct loads on each section of blade for viscous and compressible effects

I. This file has tabulated lists of $C_{l}, C_{d}$ and $C_{M}$ for each angle of attack and Mach number

\section{A.2 GENUVP Flowchart}

A flowchart of the GENUVP program structure is presented in this part. The flowchart's application is limited to the aerodynamic component. 
Summary

Program Initialization

In each time step followings

would be repeated:

- Potential Calculations

- Vortical Calculations
Start Program

Program

Initialization

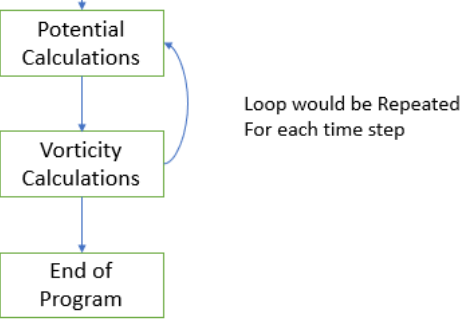


Program Initialization

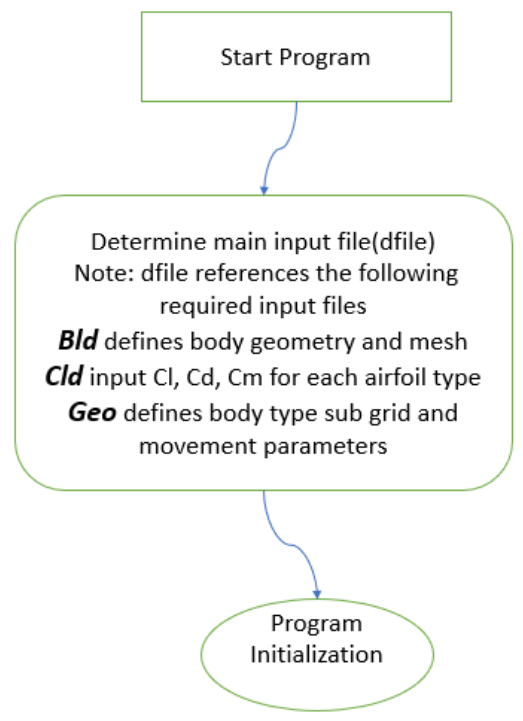

Program Initialization

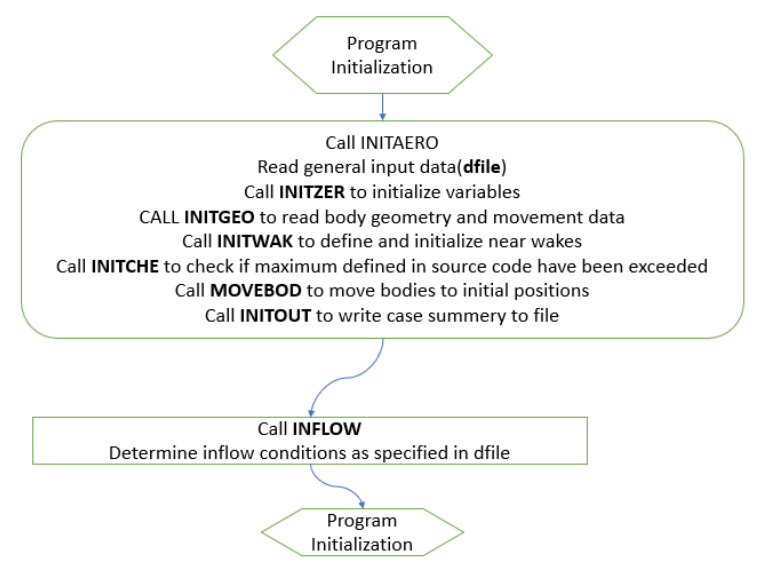

3 
Program Initialization

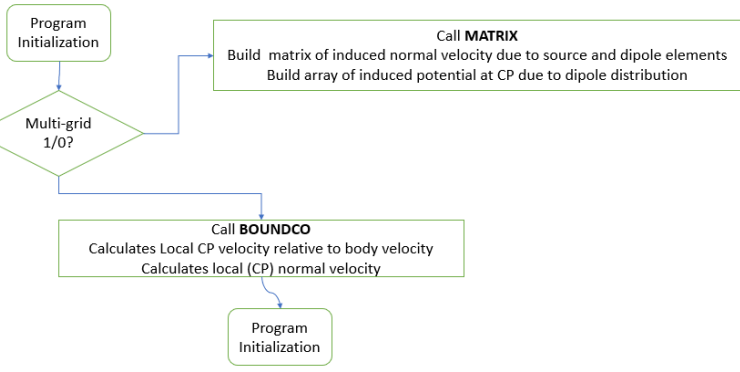

4

\section{Program Initialization}

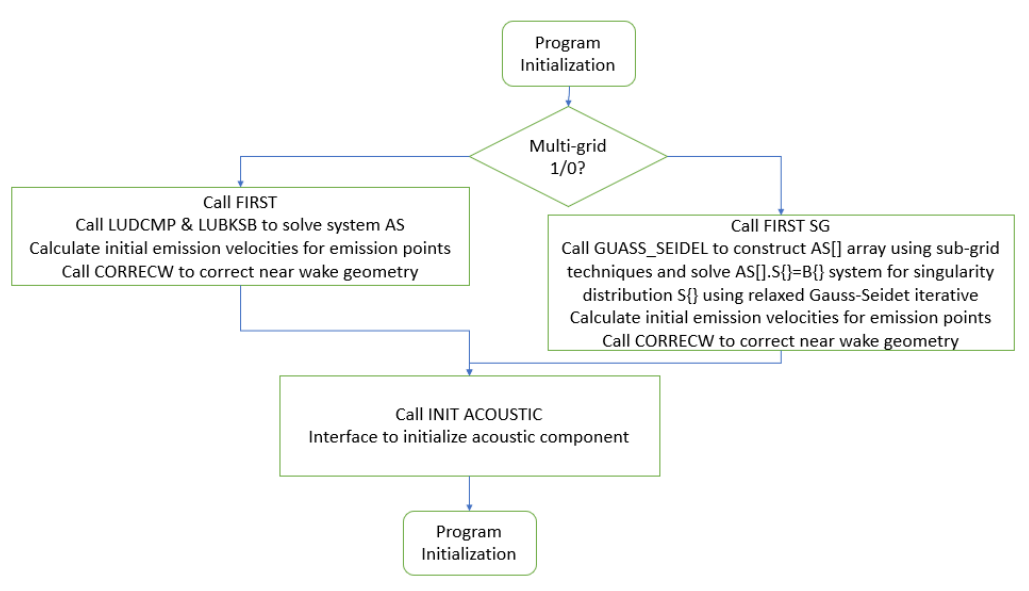

5

Program Initialization

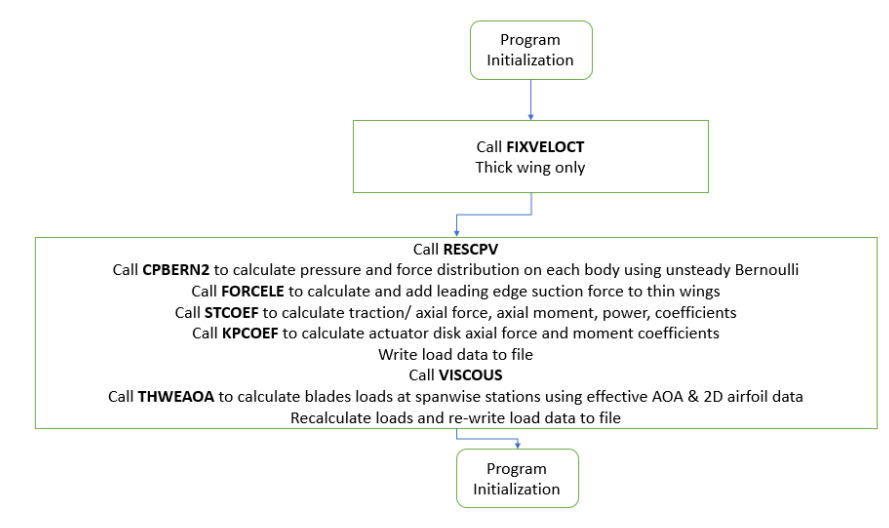

6 
Program Initialization

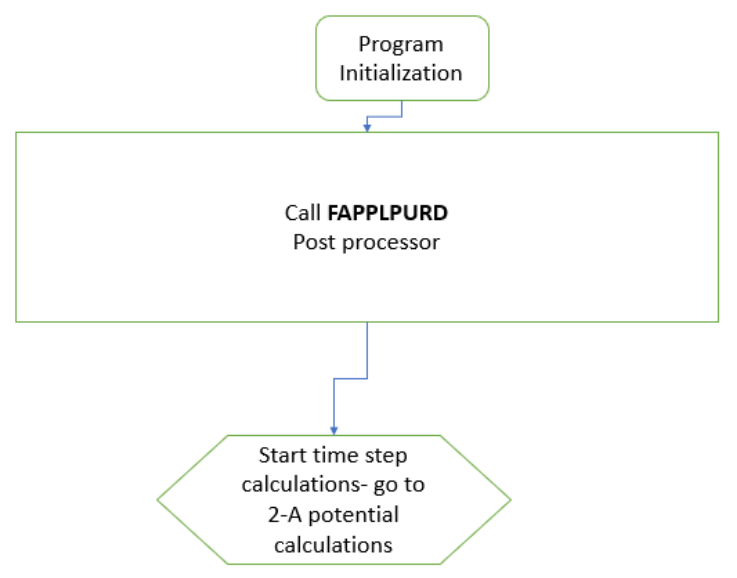




\section{Appendix B Studying Aeroacoustics Formulations}

\section{B.1 Origin of Formulation 1C for Non-Penetrable Surfaces}

The FW-H acoustic analogy covers acoustic monopole and dipole source distributions across the body surface and a quadrupole source distribution in the volume around the body for a moving solid body submerged in a fluid without mean flow. The FW-H equation was first proposed in [96] and written in [91] as:

$$
\square^{2} \dot{p}(x, t)=\frac{\partial}{\partial t}\left[\rho_{0} v_{n} \delta(f)\right]-\frac{\partial}{\partial x_{i}}\left[p \widehat{n_{l}} \delta(f)\right]+\frac{\partial^{2}}{\partial x_{i} \partial x_{j}}\left[H(f) T_{i j}\right]
$$

In the above equation, $\square^{2}$ is described as wave or $\mathrm{D}^{\prime}$ Alembertian operator in threedimensional space, $\dot{p}$ can be defined as the acoustic or perturbation pressure for an observer distanced $\mathrm{x}$ at time $\mathrm{t}$, also $v_{n}$ is defined as the element of velocity with a direction normal to the body, and $p$ is defined as the local gauge pressure on the surface of body.

$f \equiv f(y)$ is defined as the body surface equation in which $\mathrm{f}<0$ is used for inside the surface, $\mathrm{f}>0$ is used for outside of surface and $\mathrm{f}=0$ is utilized for on the surface. $\hat{n}=\nabla f$ is defined as the unit outward normal of surface, moreover $\mathrm{H}(\mathrm{f})$ and $\delta(f)$ are defined as the Heaviside and Dirac delta functions.

The first three terms on the right side of the equation (B.1) are defined as the thickness noise contribution to acoustic pressure, loading noise contribution and quadrupole noise contribution, respectively.

A data (nonphysical) surface that covers the solid surface and any volume quadrupoles producing considerable noise, that is an example of a surface described by $f$, might be 
termed penetrable. According to [63], [91], FW-H equation for the aforementioned penetrable data surface is defined as:

$$
\square^{2} \dot{p}(x, t)=\frac{\partial}{\partial t}\left[Q_{n} \delta(f)\right]-\frac{\partial}{\partial x_{i}}\left[L_{n i} \delta(f)\right]+\frac{\partial^{2}}{\partial x_{i} \partial x_{j}}\left[H(f) T_{i j}\right],
$$

In which,

$$
\begin{gathered}
Q_{n}=\rho\left(u_{n}^{\prime}-v_{n}\right)+\rho_{0}\left(v_{n}\right), \\
L_{n i}=\rho \dot{u}_{i}\left(u_{n}^{\prime}-v_{n}\right)+P_{i j} \widehat{n}_{j}, \\
P_{i j}=p \delta_{i j}-\sigma_{i j} .
\end{gathered}
$$

$T_{i j}$ is considered as the Lighthill's stress tensor. In the situations that the quadrupole source is ignored or could not be solved directly, Eq. (B.2) third's term on the right-hand side can be ignored.

In the situations that an impenetrable data surface like a solid body exists, following equation is valid.

$$
u_{n}^{\prime}-v_{n}=0
$$

So, first term in the right side of equations (B.3) and (B.4) would be omitted. Based on [91], viscous shear forces that apply on the data surface and described as $\sigma_{i j}$ would be ignored and following equations are obtained:

$$
\begin{gathered}
Q_{n}=\rho_{0} v_{n}, \\
L_{n i}=\dot{p} \delta_{i j} \widehat{n}_{j}=\dot{p} \widehat{n}_{i} .
\end{gathered}
$$


Aforementioned terms are correlated to the $Q_{n}$ and $L_{n i}$ in the convective FW-H equation presented by Najafi-Yazdi et al. [63] by:

$$
\begin{aligned}
Q_{n} & =Q_{i} \widehat{n}_{i}, \\
L_{n i} & =L_{i j} \widehat{n}_{i},
\end{aligned}
$$

In which,

$$
\begin{gathered}
Q_{i}=\rho\left(\dot{u}_{i}+U_{\infty i}-v_{i}\right)+\rho_{0}\left(v_{i}-U_{\infty i}\right), \\
L_{i j}=\rho \dot{u}_{i}\left(\dot{u}_{j}+U_{\infty j}-v_{j}\right)+P_{i j} .
\end{gathered}
$$

Non-penetration condition for the convective formulation needs:

$$
\left(u_{\imath}+U_{\infty i}-v_{i}\right) \widehat{n_{l}}=0
$$

In this case normal section of the first terms in the equations for $Q_{i}$ and $L_{i j}$ would be equal to zero and thus equal to $Q_{n}$ and $L_{n i}$ in equations (B.9) and (B.10).

$Q_{n}$ and $L_{n i}$ can be replaced in the FW-H equation using the aforementioned relationships. Applying the changes, FW-H equation would be:

$$
\dot{p}(x, t)=\frac{\partial}{\partial t} \int_{f=0}\left[\frac{Q_{n}}{4 \pi|x-y|}\right]_{r e t} d s-\frac{\partial}{\partial x_{i}} \int_{f=0}\left[\frac{L_{n i}}{4 \pi|x-y|}\right]_{r e t} d S .
$$

In the above equation, ret indicates that the related equation would be calculated at the emission time and position and $\mathrm{y}$ shows the location of the aeroacoustics source and $\mathrm{x}$ indicates the location of the observer.

The equations, for calculating the loading and thickness acoustic pressures in a subsonic mean flow in the positive $x_{1}$ direction, are obtained by utilizing the same method as the one used in [63]. 


$$
\begin{array}{r}
4 \pi \dot{p}_{T}=\left[\frac{\partial}{\partial t}+U_{\infty} \frac{\partial}{\partial x_{1}}\right] \int_{-\infty}^{t} \int_{\mathbb{R}^{3}} Q_{n} \delta(f) \frac{\delta(g)}{R^{*}} d^{3} y d \tau, \\
4 \pi \dot{p}_{L}=-\frac{\partial}{\partial x_{i}} \int_{-\infty}^{t} \int_{\mathbb{R}^{3}} L_{n i} \delta(f) \frac{\delta(g)}{R^{*}} d^{3} y d \tau,
\end{array}
$$

while $g=\tau-t+R / c_{0}$.

Thickness noise contribution to sound pressure can be calculated using the following equation [63]:

$$
\begin{aligned}
4 \pi \dot{p}_{T}=\int_{f=0} & {\left[\frac{\dot{Q_{n}}}{R^{*}\left(1-M_{R}\right)^{2}}\right]_{r e t} d \eta+\int_{f=0}\left[\frac{-\partial R^{*}}{\partial \tau} \frac{Q_{n}}{R^{* 2}\left(1-M_{R}\right)^{2}}\right]_{r e t} d \eta } \\
& +\int_{f=0}\left[\frac{Q_{n}}{R^{*}\left(1-M_{R}\right)^{3}} \frac{\partial M_{R}}{\partial \tau}\right]_{r e t} d \eta \\
& -M_{\infty} \int_{f=0}\left[\frac{\dot{\widetilde{R_{1}}} Q_{n}+\widetilde{R}_{1} \dot{Q}_{n}}{R^{*}\left(1-M_{R}\right)^{2}}\right]_{r e t} d \eta \\
& +M_{\infty} \int_{f=0}\left[\frac{\partial R^{*}}{\partial \tau} \frac{\widetilde{R_{1}} Q_{n}}{R^{* 2}\left(1-M_{R}\right)^{2}}\right]_{r e t} d \eta \\
& -M_{\infty} \int_{f=0}\left[\frac{\partial M_{R}}{\partial \tau} \frac{\widetilde{R_{1}} Q_{n}}{R^{*}\left(1-M_{R}\right)^{3}}\right]_{r e t} d \eta \\
& -U_{\infty} \int_{f=0}\left[\frac{\widetilde{R_{1}} Q_{n}}{\widetilde{R^{* 2}\left(1-M_{R}\right)}}\right]_{r e t} d \eta .
\end{aligned}
$$

Also, loading noise contribution can be calculated using the following equation: 


$$
\begin{aligned}
4 \pi p_{l}=\frac{1}{c_{0}} \int_{f=0} & {\left[\frac{\dot{L}_{n_{i}} \widetilde{R}_{i}+L_{n i} \dot{\tilde{R}}_{i}}{R^{*}\left(1-M_{R}\right)^{2}}\right]_{r e t} d \eta } \\
& -\frac{1}{c_{0}} \int_{f=0}\left[\frac{\partial R^{*}}{\partial \tau} \frac{L_{n i} \widetilde{R}_{i}}{R^{*^{2}}\left(1-M_{R}\right)^{2}}\right]_{r e t} d \eta \\
& +\frac{1}{c_{0}} \int_{f=0}\left[\frac{\partial M_{R}}{\partial \tau} \frac{L_{n i} \widetilde{R}_{i}}{R^{*}\left(1-M_{R}\right)^{3}}\right]_{r e t} d \eta \\
& +\int_{f=0}\left[\frac{L_{n i} \widetilde{R}_{i}^{*}}{R^{* 2}\left(1-M_{R}\right)}\right]_{r e t} d \eta
\end{aligned}
$$

Parameters that include dots are showing temporal derivatives with respect to the source time $\tau$ and can be computed numerically or analytically. Also vector $\eta$ is showing all the points which are located on the surface in the surface's local coordinate system.

In the situations which data surface is connected to a rigid body having an angular velocity of $\Omega$, following analytic equation can be used to compute the unit normal vector on the surface.

$$
\dot{\hat{n}}=\Omega \times \hat{n}=\epsilon_{i j k} \Omega_{j} \hat{n}_{k}
$$

In the above equation, $\epsilon_{i j k}$ is defined as the Levi-Civita symbol. Using the following equations, source temporal derivatives of $Q_{n}$ and $L_{n i}$ can be computed.

$$
\begin{gathered}
\dot{Q_{n}}=\frac{\partial}{\partial \tau}\left[\rho_{0}\left(v_{i}-U_{\infty i}\right)\right] \hat{n}_{i}+\rho_{0}\left(v_{i}-U_{\infty i}\right) \epsilon_{i j k} \Omega_{j} \hat{n}_{k}, \\
\dot{L}_{n i}=\frac{\partial \dot{p}}{\partial \tau} \hat{n}_{i}+\dot{p} \epsilon_{i j k} \Omega_{j} \hat{n}_{k},
\end{gathered}
$$


Thickness and loading noise equations can be changed to fit the panel approach application, in which data surface is divided into $\mathrm{N}$ flat panels with equal values of perturbation pressure $\dot{p}$ and surface velocity $\mathrm{v}$ in all panel area $\mathrm{S}$ containing a unit normal vector of $\hat{n}$ and centroid at point $y$. Following equations are the convective non-penetrable version of the thickness and loading noise which are obtained by focusing the surface noise contribution of each panel to originate from their centroids.

$$
\begin{aligned}
4 \pi \dot{p}_{T}\left(x, t_{a d v}\right) & =\sum_{K=1}^{N}\left[\left(\frac{\dot{Q_{n}}}{R^{*}\left(1-M_{R}\right)^{2}}-\frac{\partial R^{*}}{\partial \tau} \frac{Q_{n}}{R^{* 2}\left(1-M_{R}\right)^{2}}\right.\right. \\
& \left.+\frac{Q_{n}}{R^{*}\left(1-M_{R}\right)^{3}} \frac{\partial M_{R}}{\partial \tau}\right) \\
& -M_{\infty}\left(\frac{\dot{\widetilde{R}}_{1} Q_{n}+\tilde{R}_{1} \dot{Q}_{n}}{R^{*}\left(1-M_{R}\right)^{2}}-\frac{\partial R^{*}}{\partial \tau} \frac{\widetilde{R}_{1} Q_{n}}{R^{* 2}\left(1-M_{R}\right)^{2}}\right. \\
& \left.\left.+\frac{\partial M_{R}}{\partial \tau} \frac{\widetilde{R}_{1} Q_{n}}{R^{*}\left(1-M_{R}\right)^{3}}\right)-U_{\infty}\left(\frac{\widetilde{R}_{1}^{*} Q_{n}}{R^{* 2}\left(1-M_{R}\right)}\right)\right]_{k} S_{k} .
\end{aligned}
$$

Moreover,

$$
\begin{aligned}
& 4 \pi \dot{p}_{l}\left(x, t_{a d v}\right)=\sum_{k=1}^{N}\left[\frac { 1 } { c _ { 0 } } \left(\frac{L_{n i} \tilde{R}_{i}+L_{n i} \dot{\tilde{R}}_{i}}{R^{*}\left(1-M_{R}\right)^{2}}-\frac{\partial R^{*}}{\partial \tau} \frac{L_{n i} \tilde{R}_{i}}{R^{* 2}\left(1-M_{R}\right)^{2}}+\right.\right. \\
& \left.\left.\frac{\partial M_{R}}{\partial \tau} \frac{L_{n i} \tilde{R}_{i}}{R^{*}\left(1-M_{R}\right)^{3}}\right)+\frac{L_{n i} \tilde{R}_{i}^{*}}{R^{* 2}\left(1-M_{R}\right)}\right]_{k} S_{k} .
\end{aligned}
$$


In the equations B.22 and B.23, the right-side terms are calculated at the emission source time $\tau_{e}$. Observer time can be calculated using the following equation which utilize the acoustic radiation distance $\mathrm{R}$ at the emission source time

$$
t_{a d v}=\tau_{e}+\frac{R}{C_{0}}
$$

